\title{
HIPOELITICIDADE GLOBAL \\ PARA OPERADORES DE \\ SEGUNDA ORDEM
}

Sérgio Luis Zani

Orientador: Prof. Dr. Adalberto Panobianco Bergamasco

Dissertação apresentada ao Instituto de Ciências Matemāticas de São Carlos, da Universidade de são Paulo, para a obten ção do Título de Mestre em Matemática. 
Aos meus

pais. 
- Ao Prof. Dr. Adalberto Panobianco Bergamasco pela orientação e pelo apoio sempre presentes.

- Aos Professores do Instituto de Ciências Matemāticas de São carlos e do Departamento de Matemātica da Universidade Federal de são carlos, principalmente ao Prof. Dr. Hermano de Souza Ribeiro e ao Prof. Dr. Arnaldo Simal do Nascimento.

- Aos amigos e colegas de graduação e pós-graduação.

- Ao excelente trabalho de datilografia realizado pela secreta ria de Trabalhos Cientificos do ICMSC-USP. 
Our main aim in this work is to study the regularity of the periodic solutions of differential equations (especially second order ones) using the characterization of periodic distributions and of smooth periodic functions by means of their Fourier series. 


\section{N D I C E}

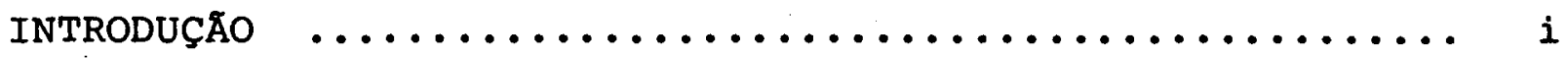

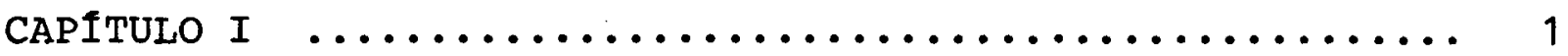

I.1. - $\mathrm{P}_{\mathrm{T}}$ : O Espaço das Funções Testes Periódicas $\ldots . \quad 2$

I.2. - $\mathrm{P}_{\mathrm{T}}^{\prime}$ : O Espaço das Distribuições Periódicas ..... 9

I.3. - T-Convolução ....................... 28

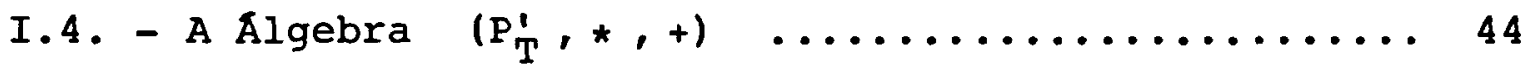

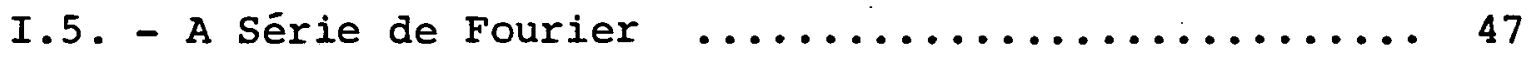

I.6. - A Série Parcial de Fourier $\ldots \ldots \ldots \ldots \ldots \ldots \ldots \ldots . \ldots . \ldots 6$

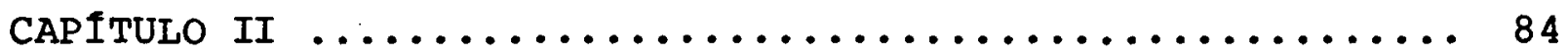

II.1. - Resolubilidade de Equações Lineares de T-Convolução .......................... 85

II.2. - Hipoeliticidade Global $\ldots \ldots \ldots \ldots \ldots \ldots \ldots \ldots \ldots . . \ldots 9$

II.3. - Exemplos de Operadores Globalmente Hipoelíticos ......................... 95

II.4. - Um Exemplo de Operador Diferencial Parcial a Coeficientes Variáveis Globalmente Hipoelítico .. 106

II.5. - Outros Exemplos .................... 112

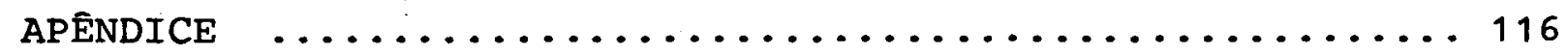

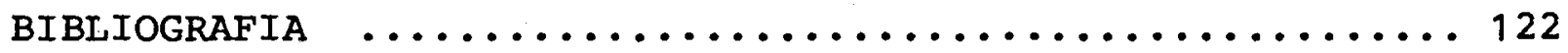




\section{N TRODU C Ã O}

Este trabalho de Mestrado tem como objetivo principal o estudo da hipoeliticidade global de certos operadores diferencí ais parciais lineares. Para chegar a 1sto fizemos no Capitulo I um estudo sobre distribuiçōes periódicas procurando tratá-las de maneira que não fosse preciso ter um conhecimento prévio da teoria das distribuições, visando com isso tornā-lo mais acessí vel. Ainda neste capítulo estudamos as séries de Fourier das distribuições periódicas e das funções periódicas infinitamente diferenciáveis de $n$ variáveis reais.

Apōs terminarmos a redação deste trabalho tomamos conhecí mento do livro Equações Diferenciais Parciais: Uma Introdução, de Rafael Iório e Valéria Iório, Projeto Euclides - 1988, o qual trata este assunto no caso de uma variável.

Os resultados obtidos neste capitulo foram a ferramenta básica para o Capítulo II, que contém a parte principal do trâ balho. Neste capítulo estudamos a influência dos termos de or dem mais baixa de um operador diferencial parcial linear a coe ficientes constantes de 2a ordem através de exemplos. Isto é, adicionamos a certos operadores homogêneos de ordem 2 outros homogêneos de ordem 1 e de ordem 0 e comparamos a hipoeliticidade do operador resultante com aquela de cada uma das parcelas.

Mostramos a hipoeliticidade do operador $\mathrm{P}$ dado por $\mathrm{Pu}=-\frac{\partial^{2}}{\partial \mathrm{x}^{2}} u-\phi^{2}(\mathrm{x}) \frac{\partial^{2}}{\partial y^{2}} u+a(x) \frac{\partial u}{\partial y}$ onde $a$ e $\phi$ são funções 
$2 \pi$-periódicas de classe $c^{\infty}, \phi(0) \neq 0$ e $\phi(x)=0$ se $\frac{\pi}{2} \leqq|x| \leqq \pi$. No caso em que $a \equiv 0, \phi(x) \equiv 1$ se $|x|<\frac{\pi}{2}$ e $\phi(x)=0$ se $\frac{3 \pi}{4} \leqq|x| \leqq \pi$ o operador $\dot{p}$ é o mesmo que foi estudado por Fujiwara e Omori ([4]).

Por fim, verificamos que para quase todo a e $[0,1]$ os operadores das familias

$$
\left(\frac{\partial}{\partial x}-a \frac{\partial}{\partial y}\right)_{a \in[0,1]} \text { e }\left(\sum_{1 \leqq} \sum_{\alpha \mid \leqq 2} a_{\alpha} D^{\alpha}+a\right)_{a \in[0,1]}
$$

são globalmente hipoeliticos.

o Teorema 4 da seção 2 e o Teorema 1 da seção 3 do Capítú lo II são de Greenfield e wallach [5]. 


\section{CAPITULO I}

o objetivo deste capitulo é caracterizar as funções perió dicas infinitamente diferenciáveis e as distribuições periódị cas com suas séries de Fourier.

Na primeira seção será feito um breve estudo envolvendo as funções periódicas infinitamente diferenciāveis.

Na segunda seção serão definidas as distribuições periōdị cas e apresentadas algumas de suas propriedades mais importan tes.

Na terceira seção será definido um tipo de convolução ( T-convolução) para funções e distribuições periódicas. Um fạ to interessante è que esta convolução fica definida para quais quer pares de distribuições periódicas, fato que não ocorre com as distribuições e convolução usuais.

Na quarta seção será dada uma condição necessāria e sufị ciente para a resolubilidade de uma equação linear da T-convolu cão.

A quinta seção será dedicada ao estudo da série de Fourier tanto das funções periōdicas infinitamente derivãveis como das distribuições periódicas. Será mostrada a caracterização de cada um destes objetos com seqüências numéricas apropriadas.

$\mathrm{Na}$ sexta seção serão estudadas as sēries parciais de Fourier para funções periódicas infinitamente diferenciáveis e distribuiçōes periódicas. 
1. $\mathrm{P}_{\mathrm{T}}$ : O ESPAÇO DAS FUNÇOES TESTES PERIODICAS

$$
\text { Sejam } T>0 \text { e } m=\left(m_{1}, \ldots, m_{n}\right) \in \mathbb{Z}^{n}
$$

DEFINIÇÃO 1. Seja

$$
P_{T}\left(\mathbb{R}^{n}\right)=\left\langle\theta \in C^{\infty}\left(\mathbb{R}^{n}\right) ; \theta(x)=\theta(x-T M), \forall x \in \mathbb{R}^{n}, \forall m \in \mathbb{Z}^{n}\right\rangle .
$$

$P_{T}\left(\mathbb{R}^{n}\right)$ ou, simplesmente, $P_{T}$ é um espaço vetorial chamado de espaço das funções testes periódicas.

DEFINIÇÃ 2 - Uma seqüēncia $\left(\theta_{j}\right)_{j \in \mathbb{N}}$ onde $\theta_{j} \in P_{T}, j \in \mathbb{N}$, converge em $P_{T}$ se existir $\theta \in P_{T}$ tal que $D^{\alpha} \theta_{j}$ converge uni formemente para $D^{\alpha} \theta$, para cada $\alpha=\left(\alpha_{1}, \ldots, \alpha_{n}\right) \in \mathbb{N}^{n}$.

Existe uma métrica em $P_{T}$ em relação a qual as sequêen cias convergentes são as da Definição 2. A saber:

$$
d(\theta, \phi)=\sum_{k=0}^{\infty} \frac{p_{k}(\theta-\phi)}{2^{k}\left(1+p_{k}(\theta-\phi)\right)},
$$

onde

$$
p_{k}(\theta)=\sup _{\substack{x \in \mathbb{R}^{n} \\|\alpha| \leqq k}}\left|D^{\alpha} \theta(x)\right|
$$

é uma semi-norma.

TEOREMA 1 - $\theta_{j}$ converge para $\theta$ em $P_{T}$ se, e somente se, $p_{k}\left(\theta_{j}-\theta\right) \rightarrow 0$ para cada $k=1,2, \ldots$. 


\section{DEMONSTRAÇÃO:}

Se $\theta_{j} \rightarrow \theta$ em $P_{T}$, então, para cada $k$,

$$
p_{k}\left(\theta_{j}-\theta\right)=\sup _{\substack{x \in \mathbb{R}^{n} \\|\alpha| \leqq k}}\left|D^{\alpha} \theta_{j}(x)-D^{\alpha} \theta(x)\right| \rightarrow 0
$$

Se $p_{k}\left(\theta_{j}-\theta\right)+0$ então para cada $\alpha \in \mathbb{N}^{n}$,

$$
\begin{aligned}
& \sup _{x \in \mathbb{R}^{n}}\left|D^{\alpha} \theta_{j}(x)-D^{\alpha} \theta(x)\right| \leqq \sup _{x \in \mathbb{R}^{n}}\left|D^{\alpha} \theta_{j}(x)-D^{\alpha} \theta(x)\right|= \\
& =p_{k}\left(\theta_{j}-\theta\right) \rightarrow 0 \text {. } \\
& |\alpha| \leqq k
\end{aligned}
$$

Para cada $\phi \in C_{C}^{\infty}\left(\mathbb{R}^{n}\right)$ defina $\theta(x)=\sum_{m \in \mathbb{Z}^{n}} \phi(x-T m)$. $\theta$ está bem definida pois, existe $C>0$ tal que $\phi(x)=0$ para todo $x$ de $\mathbb{R}^{\mathrm{n}}$ tal que $|\mathrm{x}| \geq \mathrm{C}$.

Assim, se $x \in \mathbb{R}^{n}$ e $|x| \leqq a$ para algum $a>0$ então

$$
\begin{aligned}
& \phi(\mathrm{x}-\mathrm{Tm}) \neq 0 \Rightarrow 0 \Rightarrow|\mathrm{x}-\mathrm{Tm}|<\mathrm{c} \Rightarrow\left|\mathrm{x}_{i}-\mathrm{Tm} \mathrm{m}_{i}\right|<\mathrm{c}, \\
& i=1, \ldots, \mathrm{n} \Rightarrow-\mathrm{C}<\mathrm{Tm_{i }}-\mathrm{x}_{i}<\mathrm{c}, \mathrm{i}=1, \ldots, \mathrm{n} .
\end{aligned}
$$

Como $-\mathrm{a} \leqq \mathrm{x}_{i} \leqq \mathrm{a}$ temos

$$
-c-a \leqq-c+x_{i}<\operatorname{Tm}_{i}<c+x_{i} \leqq c+a, i=1, \ldots, n .
$$

Logo, $\left|m_{i}\right| \leqq \frac{c+a}{T}, \quad i=1, \ldots, n$. Como existe ape nas um número finito de n-uplas $m$ que satisfazem esta última condição, podemos escrever

$$
\theta(x)=\phi\left(x-T m_{1}\right)+\ldots+\phi\left(x-T m_{k}\right)
$$

desde que $|x| \leqq a$. 
4

Mostremos que $\theta \in \mathbb{P}_{T}\left(\mathbb{R}^{n}\right)$.

Seja $m \in \mathbb{Z}^{n}$

$$
\begin{aligned}
& \theta(x-T m)=\sum_{k \in \mathbb{Z}^{n}} \phi(x-T m-T k)=\sum_{k \in \mathbb{Z}^{n}} \phi(x-T(m+k))= \\
& =\sum_{\ell}^{\sum} \mathbb{Z}^{n} \phi(x-T \ell)=\theta(x) . \\
& \text { Dado } x_{0} e \mathbb{R}^{n}, \text { seja a }>\text { tal que }\left|x_{0}\right|<a \text {. Assim, } \\
& \theta(x)=\phi\left(x-T m_{1}\right)+\ldots+\phi\left(x-T m_{k}\right),|x|<a
\end{aligned}
$$

e vemos que $\theta$ é infinitamente diferenciável em $x_{0}$. Logo, $\theta$ e $P_{T}\left(\mathbb{R}^{n}\right)$.

Alēm do mais,

$$
D^{\alpha} \theta(x)=D^{\alpha} \phi\left(x-T m_{1}\right)+\ldots+D^{\alpha} \phi\left(x-T m_{k}\right)
$$

e como $\phi(x-T m) \equiv 0$ se $|x|<a$ e $m \neq m_{i}, i=1, \ldots, k$, temos também que

$$
D^{\alpha} \phi(x-T m) \equiv 0 \text {. se }|x|<a \text { e } m \neq m_{i}, i=1, \ldots, n \text {. }
$$

Desse modo podemos escrever

$$
D^{\alpha} \theta(x)=\sum_{m} e^{n} D^{\alpha} \phi(x-T m)
$$

PROPOSIČÃO 1 $\cdot$ Seja $\left(\phi_{\ell}\right)_{\ell} \in \mathbb{N}, \phi_{\ell} \in C_{c}^{\infty}\left(\mathbb{R}^{n}\right), \quad \ell \in \mathbb{N}$ tal que $\phi_{\ell}$ converge para $\phi$ em $C_{C}^{\infty}\left(\mathbb{R}^{n}\right)$. Então, a seqüência $\left(\theta_{\ell}\right)_{\ell}$ e IN de $P_{T}\left(\mathbb{R}^{n}\right)$ definida por $\theta_{\ell}(x)=\sum_{m} e_{\mathbb{Z}^{n}} \phi_{\ell}(x-T m)$ converge em $P_{T}\left(\mathbb{R}^{n}\right)$, para $\theta(x)=\sum_{m e} \mathbb{Z}^{n} \phi(x-T m)$. 


\section{DEMONSTRAÇÃ:}

Existe $a>0$ tal que $s\left(\phi_{\ell}\right) \subset B[0, a]$, para todo $\ell \in \mathbb{I N}$ e $D^{\alpha} \phi_{l}$ converge para $D^{\alpha} \phi$ uniformemente para todo $\alpha \in \mathbb{I N}^{\mathrm{n}}$. Sejam $\alpha \in \mathbb{N}^{\mathrm{n}}$ e $\varepsilon>0$.

Como $D^{\alpha}\left(\phi_{\ell}-\phi\right) \in C_{c}^{\infty}\left(\mathbb{I}^{n}\right)$ existem $m_{1}, \ldots, m_{k_{\ell}}$ tais que $m e^{\sum} z^{n} D^{\alpha}\left(\phi_{l}-\phi\right)(x-T m)=D^{\alpha}\left(\phi_{l}-\phi\right)\left(x-T m_{1}\right)+\ldots+D^{\alpha}\left(\phi_{l}-\phi\right)\left(x-T m_{k_{l}}\right)$, $\mathrm{x} \in[0, \mathrm{~T}]^{\mathrm{n}}$

Mas $\phi_{\ell}(x) \equiv 0$ se $x \notin B[0, a], \quad \ell$ e $\mathbb{N}$.

Logo, se $\phi_{\ell}(x-T m) \neq 0$ então $|x-T m| \leqq a$ e isto impli ca em $x_{i}-a \leqq T m_{i} \leqq a+x_{i}$.

Como $x \in[0, T]^{n}$, vem que

$-a-T \leqq-a \leqq T m_{i} \leqq a+x_{i} \leqq a+T$

e, portanto

$$
\left|m_{i}\right| \leqq \frac{a}{T}+1
$$

Como a independe de $\ell \in \mathbb{N}$, concluímos que só existe um número finito de índices $m \mid \phi_{\ell}(x-T m) \neq 0$ para todo $\ell \in \mathbb{N}$. Desse modo, podemos escrever

$$
\begin{aligned}
& \sum_{m \in \mathbb{Z}^{n}} D^{\alpha}\left(\phi_{\ell}-\phi\right)(x-T m)=D^{\alpha}\left(\phi_{\ell}-\phi\right)\left(x-T m_{1}\right)+\ldots \\
& \ldots+D^{\alpha}\left(\Phi_{\ell}-\phi\right)\left(x-T m_{k}\right), \forall \ell e \mathbb{N}, \forall x \in[0, T]^{n} .
\end{aligned}
$$

Como $D^{\alpha} \phi_{l}$ converge para $D^{\alpha} \phi$ uniformemente, existe $\ell_{0} e \mathbb{I N}$ tal que $\ell \geqq \ell_{0}$ implica em 
6

$\sup _{x \in \mathbb{R}^{n}}\left|D^{\alpha} \phi_{\ell}(x)-D_{\phi}^{\alpha}(x)\right|<\frac{\varepsilon}{k}$.

Logo, se $\ell \geq \ell_{0}$ e $x$ e $[0, T]^{n}$

$\left|D^{\alpha} \theta_{\ell}(x)-D^{\alpha} \theta(x)\right|=\left|D^{\alpha}\left(\theta_{\ell}-\theta\right)(x)\right|=$

$=\left|D^{\alpha}\left(\sum_{m \in \mathbb{Z}^{n}}\left(\phi_{\ell}-\phi\right)(x-T m)\right)\right|=\left|\sum_{m \in \mathbb{Z}^{n}} D^{\alpha}\left(\phi_{\ell}-\phi\right)(x-T m)\right|:$

$=\left|D^{\alpha}\left(\phi_{\ell}-\phi\right)\left(x-T m_{1}\right)+\ldots+D^{\alpha}\left(\phi_{\ell}-\phi\right)\left(x-T m_{k}\right)\right| \leqq$

$\leqq\left|D^{\alpha}\left(\phi_{\ell}-\phi\right)\left(x-T m_{1}\right)\right|+\ldots+\left|D^{\alpha}\left(\phi_{\ell}-\phi\right)\left(x-T m_{k}\right)\right|<$

$<\frac{\varepsilon}{\mathrm{k}}+\ldots+\frac{\varepsilon}{\mathrm{k}}=\varepsilon$.

Considere

$U_{T}\left(\mathbb{R}^{n}\right)=\left\{\xi e c_{c}^{\infty}\left(\mathbb{R}^{n}\right), \sum_{m \in \mathbb{Z}^{n}} \xi(x-T m)=1, \forall x \in \mathbb{R}^{n}\right\}$.

Se $\xi e U_{T}\left(\mathbb{R}^{n}\right)$ diremos que $\xi$ é unitária.

Um exemplo de função unitária é $\xi: \mathbb{R} \rightarrow \mathbb{R}$ dada por

$\xi(t)=\left\langle\begin{array}{ll}\int_{|t|}^{1} \exp \left(\frac{-1}{x(1-x)}\right) d x & \text { se }|t|<1 \\ \int_{0}^{1} \exp \left(\frac{-1}{x(1-x)}\right) d x & \text { se }|t| \geqq 1\end{array}\right.$

Verifica-se que $\xi \in C^{\infty}(\mathbb{R})$ e $S(\xi)=[-1,1]$.

Dado $t \in \mathbb{R}$ seja $n_{0}$ e $\mathbb{Z}$ tal que $n_{0} \leqq t \leqq n_{0}+1$ 
isto é, $0 \leq t-n_{0} \leq 1$ ou $-1 \leq n_{0}-t \leq 0$.

Se $n \geqq n_{0}+2$ então $t \leqq n$ e

$|t-n|=n-t \geq n_{0}-t+2 \geq-1+2=1$

logo, $\xi(t-n)=0$.

Se $\mathrm{n} \leqq \mathrm{n}_{0}-1$ então $\mathrm{n}<\mathrm{t}$ e

$|t-n|=t-n \geqq t+1-n_{0}=\left(t-n_{0}\right)+1 \geqq 1$

logo, $\xi(t-n)=0$.

Assim,

$\sum_{n=-\infty}^{+\infty} \xi(t-n)=\xi\left(t-n_{0}\right)+\xi\left(t-n_{0}-1\right)=$

$=\left(\int_{0}^{1} \exp \left(\frac{-1}{x(1-x)}\right) d x\right)^{-1} \cdot\left[\int_{t-n_{0}}^{1} \exp \left(\frac{-1}{x(1-x)}\right) d x+\right.$

$\left.+\int_{1+n_{0}-t}^{1} \exp \left(\frac{-1}{x(1-x)}\right) d x\right]=\left(\int_{0}^{1} \exp \left(\frac{-1}{x(1-x)}\right) d x\right)^{-1}$.

- $\left[\int_{t-n_{0}}^{1} \exp \left(\frac{-1}{x(1-x)}\right) d x-\int_{t_{0}-n_{0}}^{0} \exp \left(\frac{-1}{x(1-x)}\right) d x\right]=$

$=\left(\int_{0}^{1} \exp \left(\frac{-1}{x(1-x)}\right) d x\right)^{-1} \cdot\left(\int_{0}^{1} \exp \left(\frac{-1}{x(1-x)}\right) d x\right)=1$.

Se $\xi \in U_{T}\left(\mathbb{R}^{n}\right)$ então $\xi \in C_{C}^{\infty}\left(\mathbb{R}^{n}\right)$ e como visto

$\sum_{m} \stackrel{\mathbb{Z}^{n}}{D^{\alpha} \xi(x-T m)}=D^{\alpha} \sum_{m} e_{\mathbb{Z}^{n}} \xi(x-T m)=D^{\alpha} 1=0$. 
$\underline{\text { PROPOSIÇAO 2 }}$. Se $\left(\theta_{j}\right)$ converge para $\theta$ em $\quad P_{T}\left(\cdot \mathbb{R}^{n}\right) \quad$ e $\xi \in U_{T}\left(\mathbb{R}^{n}\right)$ então $\left(\xi \cdot \theta_{j}\right)$ converge para $\xi \cdot \theta$ em $C_{C}^{\infty}\left(\mathbb{R}^{n}\right)$.

\section{DEMONSTRAÇÃ:}

i) $s\left(\xi . \theta_{j}\right) \subset s(\xi)$

ii) Dado $\alpha e \mathbb{N}^{n}$ temos que

$$
D^{\alpha}\left(\xi \cdot \theta_{j}\right)=\sum_{\gamma+\beta=\alpha} \frac{\alpha !}{\gamma ! \beta !} D^{\gamma} \xi \cdot D^{\beta} \theta_{j}
$$

converge uniformemente para

$\sum_{\gamma+\beta=\alpha} \frac{\alpha !}{\gamma ! \beta !} D^{\gamma} \xi D^{\beta} \theta=D^{\alpha}(\xi . \theta)$

pois $\theta_{j}$ converge para $\theta$ em $P_{T}\left(\mathbb{R}^{n}\right)$.

A proposição seguinte mostra que é sempre possivel escre ver uma funcão $\theta$ de $P_{T}$ na forma $\sum_{m} e_{\mathbb{Z}^{n}} \phi(x-T m)$ com $\phi e C_{C}^{\infty}$.

$\underline{\text { PROPOSIÇÃO 3 }}$ - A aplicação

$$
\begin{gathered}
\tilde{T}: C_{C}^{\infty}\left(\mathbb{R}^{n}\right) \longrightarrow P_{T}\left(\mathbb{R}^{n}\right) \\
\phi \longmapsto \tilde{T} \phi
\end{gathered}
$$

onde $\quad \tilde{\mathrm{T}} \phi(\mathrm{x})=\sum_{\mathrm{m} \in \mathbb{Z}^{\mathrm{n}}} \phi(\mathrm{x}-\mathrm{Tm}) \quad$ é sobrejetora.

\section{DEMONSTRAÇÃO:}

Dada $\theta \in P_{T}\left(\mathbb{R}^{n}\right)$ defina $\phi=\xi \cdot \theta \in C_{C}^{\infty}\left(\mathbb{R}^{n}\right)$ onde $\xi \in U_{T}\left(\mathbb{R}^{n}\right)$. Assim, 


$$
\begin{aligned}
& (T \phi)(x)=\sum_{m \in \mathbb{Z}^{n}} \phi(x-T m)=\sum_{m \in \mathbb{z}^{n}} \xi(x-T m) \cdot \theta(x-T m)= \\
& =\theta(x) \sum_{m e^{n}} \xi(x-T m)=\theta(x) .
\end{aligned}
$$

Logo,

$\mathbf{T} \phi=\theta$

Note $\xi$ é uma função unitäria arbitrária.

2. $\mathrm{P}_{\mathrm{T}}^{\prime}$ : O ESPAÇO DAS DISTRIBUIÇŌES PERIODICAS

DEFINIÇÃO 1 U Um funcional linear $f: P_{T}\left(\mathbb{R}^{n}\right) \rightarrow \mathbb{C}$ é dito contínuo (ou seqüencialmente contínuo) se $\left(\theta_{j}\right)$ converge para 0 em $P_{T}$ implica que $f\left(\theta_{j}\right)$ convirja para 0 em $\mathbb{C}$.

DEFINIÇÃo 2 . O espaço vetorial $P_{T}^{\prime}\left(\mathbb{R}^{n}\right)=\{f ; f$ é funcio nal linear contínuo de $\left.P_{T}\left(\mathbb{R}^{n}\right)\right\}$ é chamado de espaço das dis tribuições periódicas e f é dita uma distribuigão periódica.

Se $f \in P_{T}^{\prime}$ e $\theta \in P_{T}$ usaremos a seguinte notação:

$$
f(\theta)=(f, \theta)
$$

TEOREMA $1:$ Seja $f: P_{T}\left(\mathbb{R}^{n}\right) \rightarrow \mathbb{C}$ um funcional linear. são $\mathrm{e}$ quivalentes:

i) $\mathrm{f}$ è contínuo; 
ii) Existem $c>0$ e $m \in \mathbb{N}$ tais que

$$
|(f, \theta)| \leqq C \quad \sum_{|\alpha| \leqq m} \sup _{x \in \mathbb{R}^{n}}\left|D^{\alpha} \theta(x)\right|,
$$

para toda $\theta$ de $\mathrm{P}_{\mathrm{T}}$.

\section{DEMONSTRAÇÃ:}

Suponha que (ii) seja válido. Se $\left(\theta_{j}\right)$ vai a 0 em $P_{T}$ então $\sup _{x \in \mathbb{R}^{n}}\left|D^{\alpha} \theta_{j}(x)\right|$ tende a zero para todo $\alpha \in \mathbb{N}^{n}$. Logo, a soma

$$
|\alpha| \leqq m \sup _{x \in \mathbb{R}^{n}}\left|D^{\alpha} \theta_{j}(x)\right|
$$

também tende a zero quando $j$ terde a infinito.

Portanto, usando a desigualdade em (ii) concluímos que $f$ è continuo.

Suponha agora que $f$ è contínuo.

Se $f \equiv 0$ a implicação é imediata. Admitamos que (ii) seja falso e $f \not 0$.

Então, para cada $m \in \mathbb{N}$, podemos encontrar $\tilde{\theta}_{m} \in P_{T}$ tal que

$$
\begin{aligned}
& \left|\left(f, \tilde{\theta}_{m}\right)\right|>m\left|\sum_{\leqq m} \sup _{x \in \mathbb{R}^{n}}\right| D^{\alpha} \tilde{\theta}_{m}(x) \mid \\
& \text { Definindo } \theta_{m}=\frac{\tilde{\theta} m}{\left|\left(f, \tilde{\theta}_{m}\right)\right|} \text { temos que } \\
& \left|\left(f, \theta_{m}\right)\right|=1>m \sum_{|\alpha| \leqq m} \sup _{x \in \mathbb{R}^{n}}\left|D^{\alpha} \theta_{m}(x)\right|
\end{aligned}
$$

E daí, 
$0<\sum_{|\alpha| \leqq m} \sup _{x \in \mathbb{R}^{n}}\left|D^{\alpha} \theta_{m}(x)\right|<\frac{1}{m}$

Assim, $\theta_{m}$ converge pare. 0 em $P_{T}$, porém $\left(f, \theta_{m}\right)$ não converge a zero em $\mathfrak{C}$. Absurdo.

DEFINIÇÃO 3. Diremos que uma seqüência $\left(f_{n}\right)$ de distribuições T-periódicas é convergente em $P_{T}^{\prime}\left(\mathbb{R}^{n}\right)$ se existir f e $P_{T}^{\prime}\left(\mathbb{R}^{n}\right)$ tal que para toda $\theta \in P_{T}\left(\mathbb{R}^{n}\right)$ a seqüência $\left(f_{n}, \theta\right)$ converge $p \underline{a}$ ra $(f, \theta)$.

o próximo teorema mostra que existe uma identificação en tre o $P_{T}^{\prime}\left(\mathbb{R}^{n}\right)$ e o subespaço vetorial e topológico (munido da topologia induzida de $D^{\prime}\left(\mathbb{R}^{n}\right)$ definido por:

$$
D_{T}^{\prime}\left(\mathbb{R}^{n}\right)=\left\{u \in D^{\prime}\left(\mathbb{R}^{n}\right) ; u=u_{T m}, \forall m \in \mathbb{Z}^{n}\right\}
$$

onde $\left\langle u_{T m}, \phi\right\rangle=\left\langle u, \phi_{-T m}\right\rangle$ e $\phi_{T m}(x)=\phi(x-T m)$.

TEOREMA 2 Existe um homeomorfismo linear entre $P_{T}^{\prime}\left(\mathbb{R}^{n}\right)$ e $\mathrm{D}_{\mathrm{T}}^{\prime}\left(\mathrm{IR}^{\mathrm{n}}\right)$.

DEMONSTRAÇÃO:

$$
\begin{aligned}
& \text { Seja } f \in P_{T}^{\prime}\left(\mathbb{R}^{n}\right) \\
& \text { Para cada } \phi \in C_{C}^{\infty}\left(\mathbb{R}^{n}\right) \text { considere } \\
& \theta=\sum_{m \in \mathbb{Z}^{n} \phi_{T m} \in P_{T}\left(\mathbb{R}^{n}\right)}
\end{aligned}
$$


1.2

$u: c_{c}^{\infty}\left(\mathbb{R}^{n}\right)+\mathbb{c}$ por $u(\phi)=\left(f, \sum_{m e^{n}} \phi_{T m}\right)=(f, \theta)$.

Mostremos que u e $D_{T}^{\prime}\left(\mathbb{R}^{n}\right)$.

Linearidade: Se $\phi, \psi$ e $C_{c}^{\infty}\left(\mathbb{I R}^{n}\right)$ e $\lambda$ e $\mathbb{C}$ então

$$
\begin{aligned}
& \mathrm{u}(\phi+\psi)=\left(\mathrm{f},{ }_{\mathrm{m}} \mathrm{e}_{\mathrm{z}^{\mathrm{n}}}(\phi+\psi)_{\mathrm{Tm}}\right)= \\
& =\left(\mathbf{f}, \sum_{m \in \mathbb{z}^{\mathrm{n}}} \phi_{\mathrm{Tm}}+\sum_{\mathrm{m}} \mathrm{z}^{\mathrm{n}} \Psi_{\mathrm{Tm}}\right)= \\
& =\left(f, \sum_{m \in \mathbb{Z}^{n}} \phi_{T m}\right)+\left(f, \sum_{m \in \mathbb{Z}^{n}} \psi_{T m}\right)=u(\phi)+u(\psi)
\end{aligned}
$$

e

$$
\begin{aligned}
& u(\lambda \phi)=\left(f, \sum_{m} e_{\mathbb{Z}^{n}}(\lambda \phi)_{T m}\right)=\left(f, \sum_{m \mathbb{Z}^{n}}{ }^{\lambda \phi} \operatorname{Tm}\right)= \\
& =\left(\mathbf{f}, \lambda \sum_{m \in \mathbb{Z}^{n}} \phi_{T m}\right)=\lambda\left(\mathbf{f}, \sum_{m} e_{\mathbb{Z}^{n}} \phi_{T m}\right)=\lambda u(\phi) .
\end{aligned}
$$

Continuidade: Se $\phi_{j}$ converge para zero em $C_{C}^{\infty}\left(\mathbb{R}^{n}\right)$, a Propo sição 1 da seção anterior garante que $\theta_{j}=\sum_{m e \mathbb{Z}^{n}}\left(\phi_{j}\right)_{T m}$ conver ge para $\theta=\sum_{m \in \mathbb{Z}^{\mathrm{n}}} 0_{\mathrm{Tm}}=0$ em $\mathrm{P}_{\mathrm{T}}\left(\mathbb{R}^{\mathrm{n}}\right)$.

Desse modo, $u\left(\phi_{j}\right)=\left(f, \theta_{j}\right)$ converge para 0 em $\mathbb{C}$. Logo, $u \in D^{\prime}\left(\mathbb{R}^{n}\right)$. Usaremos a notação $u(\phi)=\langle u, \phi\rangle$.

Periodicidade: Se $\phi \in C_{c}^{\infty}\left(\mathbb{R}^{n}\right)$ e $m \in \mathbb{Z}^{n}$ então

$$
\begin{aligned}
& \left\langle u_{T m}, \phi\right\rangle=\left\langle u, \phi_{-T m}\right\rangle=\left(f, \sum_{k} e_{z^{n}}\left(\phi_{-T m}\right)_{T k}\right)^{\prime}= \\
& \left(\mathrm{f}, \sum_{\mathrm{k}} \mathrm{e}_{\mathbb{Z}^{\mathrm{n}}} \phi_{\mathrm{T}(\mathrm{k}-\mathrm{m})}\right)=\left(\mathrm{f}, \sum_{\mathrm{k} \in \mathbb{Z}^{\mathrm{n}}} \phi_{\mathrm{Tk}}\right)=\langle\mathrm{u}, \phi\rangle,
\end{aligned}
$$

isto é, $u_{\mathrm{Tm}}=u$.

Portanto, $u \in D_{T}^{\prime}\left(\mathbb{R}^{n}\right)$. 
Seja

$$
\begin{aligned}
A: P_{T}^{\prime}\left(\mathbb{R}^{n}\right) & \longrightarrow D_{T}^{\prime}\left(\mathbb{R}^{n}\right) \\
f & \longmapsto u=T(f)
\end{aligned}
$$

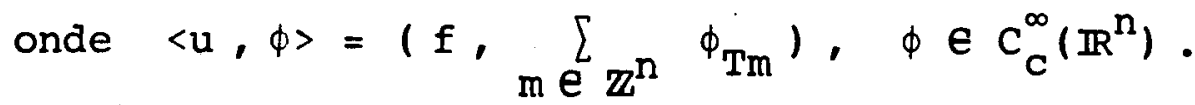

Mostremos que A é linear e contínuo.

Linearidade: Se $f, g \in P_{T}^{\prime}\left(\mathbb{R}^{n}\right)$ e $\lambda \in \mathbb{C}$ então, para toda $\phi e c_{c}^{\infty}\left(\mathbb{R}^{n}\right)$ temos

$$
\begin{aligned}
& \langle A(f+g), \phi\rangle=\left(f+g, \sum_{m \in \mathbb{Z}^{n}} \phi_{T m}\right)= \\
& =\left(f, \sum_{m e^{n}} \phi_{T m}\right)+\left(g, \sum_{m \in \mathbb{Z}^{n}} \phi_{T m}\right)= \\
& =\langle A(f), \phi\rangle+\langle A(g), \phi\rangle=\langle A(f)+A(g), \phi\rangle
\end{aligned}
$$

e

$$
\begin{aligned}
& \langle A(\lambda f), \phi\rangle=\left\langle\lambda f, \sum_{m} \mathbb{e}_{\mathbb{Z}^{n}} \phi_{\mathrm{Tm}}\right\rangle=\lambda\left\langle\mathrm{f}, \sum_{\mathrm{m} \in \mathbb{Z}^{\mathrm{n}}} \phi_{\mathrm{Tm}}\right\rangle= \\
& =\lambda\langle A(f), \phi\rangle=\langle\lambda A(f), \phi\rangle .
\end{aligned}
$$

Continuidade: $\operatorname{seja}\left(f_{j}\right), f_{j} \in P_{T}^{\prime}\left(\mathbb{R}^{n}\right)$ tal que $f_{j}$ tende a zero em $P_{T}^{\prime}\left(\mathbb{R}^{n}\right)$.

Dada $\phi$ e $C_{C}^{\infty}\left(\mathbb{R}^{n}\right)$, temos que

$$
\lim _{j \rightarrow \infty}\left\langle A\left(f_{j}\right), \phi\right\rangle=\lim _{j \rightarrow \infty}\left(f_{j}, \sum_{m} e_{\mathbb{Z}^{n}} \phi_{T m}\right)=0 .
$$

Logo, $A\left(f_{j}\right)$ tende a zero em $D_{T}^{\prime}\left(\mathbb{R}^{n}\right)$.

Vamos agora, a partir de $u \in D_{T}^{\prime}\left(\mathbb{R}^{n}\right)$, definir uma única 
distribuiçāo T-periódica $f$ de modo que esta aplicação seja a inversa de $\mathrm{A}$.

Seja $\xi \in U_{T}\left(\mathbb{R}^{n}\right)$. Para cada $u \in D_{T}^{\prime}\left(\mathbb{R}^{n}\right)$ definamos

$$
\begin{aligned}
f_{\xi}: P_{T}\left(\mathbb{R}^{n}\right) & \longrightarrow \mathbb{C} \\
\theta & \longmapsto\langle u, \xi, \theta\rangle .
\end{aligned}
$$

Mostremos que $f_{\xi} \in P_{T}^{\prime}\left(\mathbb{R}^{n}\right)$.

Linearidade: $\operatorname{Sejam} \theta, \beta \in \mathrm{P}_{\mathrm{T}}\left(\mathbb{R}^{\mathrm{n}}\right)$ e $\lambda \in \mathbb{C}$.

i) $f_{\xi}(\theta+\beta)=\langle u, \xi(\theta+\beta)\rangle=\langle u, \xi \theta+\xi \beta\rangle=\langle u, \xi \theta\rangle+\langle u, \xi \beta\rangle=$ $=f_{\xi}(\theta)+f_{\xi}(\beta)$

ii) $f_{\xi}(\lambda \theta)=\langle u, \xi(\lambda \theta)\rangle=\langle u, \lambda \xi \theta\rangle=\langle\lambda u, \xi \theta\rangle=\lambda\langle u, \xi \theta\rangle=$ $=\lambda f_{\xi}(\theta)$.

Continuidade: $\operatorname{seja}\left(\theta_{j}\right), \theta_{j} \in P_{T}\left(\mathbb{R}^{n}\right)$ tal que $\theta_{j}$ tende a zero em $P_{T}\left(\mathbb{R}^{n}\right)$.

A Proposição 2 da secão anterior nos diz que $\left(\xi \theta_{j}\right)$ ten de a zero em $C_{C}^{\infty}\left(\mathbb{R}^{n}\right)$.

Como $u \in D_{T}^{\prime}\left(\mathbb{R}^{n}\right)$ temos que $f_{\xi}\left(\theta_{j}\right)=\left\langle u, \xi \theta_{j}\right\rangle \rightarrow 0$. Logo, $\mathrm{f}_{\xi} \in \mathrm{P}_{\mathrm{T}}^{\prime}\left(\mathbb{R}^{\mathrm{n}}\right)$.

Mostremos que a definicão de $f_{\xi}$ independe da escolha de $\xi$, isto $\bar{e}$, se $\xi, n \in U_{T}\left(\mathbb{R}^{n}\right)$ então $f_{\xi}=f_{n}$. Mostremos antes que

$$
u=\sum_{m} \mathbb{z}^{\mathrm{n}}(\mathrm{un}) \mathrm{Tm}
$$


15

Seja $\phi \in C_{c}^{\infty}\left(\mathbb{R}^{n}\right)$.

Então

$$
\begin{aligned}
& \left.\left\langle\mathrm{u}-\sum_{|\mathrm{m}|} \sum_{\leq k}(\mathrm{u})\right)_{\mathrm{Tm}}, \phi\right\rangle=\left\langle\mathrm{u}-\sum_{|\mathrm{m}|} \sum_{\leqq k} u_{\mathrm{Tm}} n_{\mathrm{Tm}}, \phi\right\rangle= \\
& =\left\langle\mathrm{u}-\sum_{|\mathrm{m}| \leqq \mathrm{k}} \mathrm{u} \eta_{\mathrm{Tm}}, \phi\right\rangle=\left\langle\mathrm{u}\left(1-\sum_{|\mathrm{m}| \leqq \mathrm{k}} \eta_{\mathrm{Tm}}\right), \phi\right\rangle= \\
& =\left\langle\mathrm{u}, \phi \cdot\left(1-\sum_{|\mathrm{m}| \leqq \mathrm{k}} n_{\mathrm{Tm}}\right)\right\rangle
\end{aligned}
$$

converge para zero pois, $1-\sum_{|\mathrm{m}| \leqq k} n_{\mathrm{Tm}}$ tende a zero em $\mathrm{P}_{T}\left(\mathbb{R}^{\mathrm{n}}\right)$ e pela Proposição 2 da seção anterior $\phi .\left(1-\sum_{|\mathrm{m}|} \sum_{\mathrm{k}} n_{\mathrm{Tm}}\right)$ ten de a zero em $c_{c}^{\infty}\left(\mathbb{R}^{n}\right)$

Além do mais, como

$$
\left\langle\sum_{|m| \leqq k}{ }^{\left.(u n)_{T m}, \phi\right\rangle=\sum_{|m| \leqq k}\left\langle(u n)_{T m}, \phi\right\rangle}\right.
$$

temos que

$$
\sum_{m} \in \mathbb{z}^{\mathrm{n}}\left\langle\left(u_{n}\right)_{\mathrm{Tm}}, \phi\right\rangle=\left\langle\sum_{\mathrm{m} \in \mathbb{Z}^{\mathrm{n}}}\left(\mathrm{un}_{\mathrm{Tm}}, \phi\right\rangle .\right.
$$

Provemos agora que $f_{\xi}=f_{n}$.

Dada $\theta \in \mathrm{P}_{\mathbf{T}}$ tome $\phi=\xi \theta$ então

$$
\begin{aligned}
& \mathbf{f}_{\xi}(\theta)=\langle u, \xi \theta\rangle=\left\langle\sum_{m \in \mathbb{Z}^{n}}(u n)_{T m}, \xi \theta\right\rangle=\sum_{m \in \mathbb{Z}^{n}}\left\langle(u n)_{T m}, \xi \theta\right\rangle=
\end{aligned}
$$

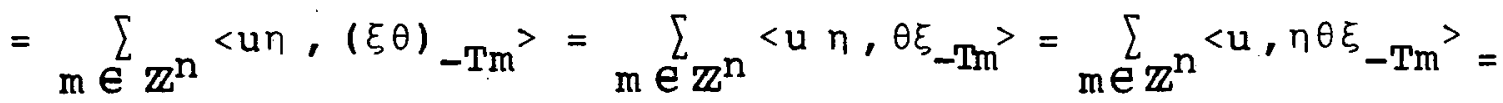

$$
\begin{aligned}
& =\sum_{m \in \mathbb{Z}^{\mathrm{n}}}\left\langle\mathrm{u} \xi_{-\mathrm{Tm}}, n \theta\right\rangle=\sum_{\mathrm{m} \in \mathbb{Z}^{\mathrm{n}}}\langle(\mathrm{u} \xi)-\mathrm{Tm}, n \theta\rangle= \\
& =\left\langle\sum_{m} e_{\mathbb{Z}^{n}}^{(u \xi)}-\mathrm{Tm}, \eta \theta\right\rangle=\langle u, n \theta\rangle=\mathrm{f}_{n}(\theta) .
\end{aligned}
$$


Passaremos a escrever somente

$$
f_{\xi}=f
$$

Definamos

$$
\begin{aligned}
H: D_{T}^{\prime}\left(\mathbb{R}^{n}\right) & \longrightarrow P_{T}^{\prime}\left(\mathbb{R}^{n}\right) \\
u & \longmapsto H(u)
\end{aligned}
$$

onde $H(u): P_{T}\left(\mathbb{R}^{n}\right) \rightarrow \mathbb{C}$

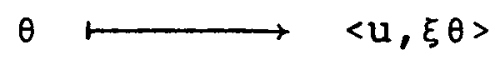

e $\quad \xi \in U_{T}$.

$H$ é linear pois, dados $u, v \in D_{T}^{\prime}\left(\mathbb{R}^{n}\right)$ e $\lambda$ e $\mathbb{C}$ temos pa ra todo $\theta \in P_{T}\left(\mathbb{R}^{n}\right)$

i) $(\mathrm{H}(\mathrm{u}+\mathrm{v}), \theta)=\langle\mathrm{u}+\mathrm{v}, \xi \theta\rangle=\langle\mathrm{u}, \xi \theta\rangle+\langle\mathrm{v}, \xi \theta\rangle=$

$$
=(H(u), \theta)+(H(v), \theta)=(H(u)+(H(v), \theta) ;
$$

ii) $(H(\lambda u), \theta)=\langle\lambda u, \xi \theta\rangle=\lambda\langle u, \xi \theta\rangle=(\lambda H(u), \theta)$.

$H$ é contínuo pois, se $u_{j} \rightarrow 0$ em $D_{T}^{\prime}\left(\mathbb{R}^{n}\right)$ então para ca da $\theta$ e $P_{T}\left(\mathbb{R}^{n}\right)$ e $\quad \xi \in U_{T}\left(\mathbb{R}^{n}\right)$

$$
\left(H\left(u_{j}\right), \theta\right)=\left\langle u_{j}, \xi \theta\right\rangle \rightarrow 0 \text {. }
$$

Portanto, $H\left(u_{j}\right) \rightarrow 0$ em $P_{T}^{\prime}\left(\mathbb{R}^{n}\right)$.

Mostremos que $H$ é a inversa de $A$.

Sejam $u \in D_{m}^{\prime}\left(\mathbb{R}^{n}\right), \phi e C_{n}^{\infty}\left(\mathbb{R}^{n}\right)$ e $\xi \in U_{m}\left(\mathbb{R}^{n}\right)$. 
Observe que se $a>0$ é tal que $S(\xi) \subset B[0, a]$ então

$$
\begin{aligned}
& \psi(x)=\xi(x) \sum_{m \in \mathbb{Z}^{n}} \phi_{T m}(x)=\sum_{m \in \mathbb{Z}^{n}} \xi(x) \phi_{T m}(x)= \\
& =\sum_{m \in \mathbb{Z}^{n}}\left(\phi \cdot \xi_{-T m}{ }^{T}(x)=0\right.
\end{aligned}
$$

se $|x|>a$ e, se $|x| \leqq a$ então

$$
\psi(x)=\xi(x)\left(\phi\left(x-T m_{1}\right)+\ldots+\phi\left(x-T m_{k}\right)\right)
$$

para certos $m_{1}, \ldots, m_{k}$ e $\mathbb{z}^{n}$.

Deste modo, para todo $x \in \mathbb{R}^{\mathrm{n}}$ a série

$$
\sum_{m \in \mathbb{Z}^{\mathrm{n}}} \xi \phi_{\mathrm{Tm}}=\sum_{\mathrm{m} \mathrm{e}^{\mathrm{n}}}(\phi \xi-\mathrm{Tm})_{\mathrm{Tm}} \quad \text { é finita. }
$$

Tendo em vista isto, obtemos

$$
\begin{aligned}
& \langle A(H(u)), \phi\rangle=\left(H(u), \sum_{m e} \mathbb{Z}^{n} \phi_{T m}\right)=\left\langle u, \xi \sum_{m}^{\sum} \mathbb{Z}^{n} \phi_{T m}>=\right. \\
& =\left\langle u, \sum_{m \in z^{n}}(\phi \xi-T m)_{T m}\right\rangle=\sum_{m} \mathbb{z}\left\langle u,\left(\phi \xi-T m{ }^{\prime}{ }_{T m}\right\rangle=\right.
\end{aligned}
$$

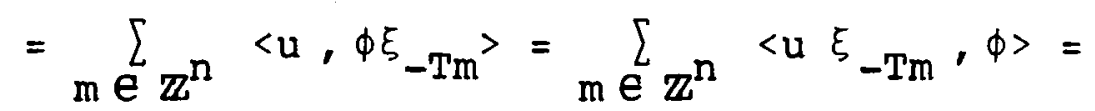

$$
\begin{aligned}
& =\sum_{m \in \mathbb{Z}^{\mathrm{n}}}\langle(\mathrm{u} \xi)-\mathrm{Tm}, \phi\rangle=\left\langle\sum_{\mathrm{m} \in \mathbb{Z}^{\mathrm{n}}}(\mathrm{u} \xi){ }_{-\mathrm{Tm}}, \phi\right\rangle=\langle\mathrm{u}, \phi\rangle
\end{aligned}
$$

onde as duas ültimas igualdades são válidas em virtude da obser vação feita antes de demonstrarmos que $f_{\eta}=f_{\xi}$.

Agora, para $f \in P_{T}^{\prime}\left(\mathbb{R}^{n}\right), \theta e P_{T}\left(\mathbb{R}^{n}\right)$ e $\xi$ e $U_{T}\left(\mathbb{R}^{n}\right)$,

$(H(A(f)), \theta)=\langle A(f), \xi \theta\rangle=\left(f, \sum_{m \in \mathbb{Z}^{n}}(\xi \theta)_{T m}\right)=$

(f, $\left.\sum_{m} e_{\mathbb{Z}^{\mathrm{n}}} \xi_{\mathrm{Tm}} \theta\right)=\left(\mathrm{f}, \theta \sum_{\mathrm{m} \in \mathbb{Z}^{\mathrm{n}}} \xi_{\mathrm{Tm}}\right)=(\mathrm{f}, \theta)$. 
Portanto, $\mathrm{H}=\mathrm{A}^{-1}$, o que demonstra o teorema.

De agora em diante não distinguiremos mais u e $f$. Ape nas quando julgarmos que possa haver dúvidas faremos alguma men ção.

Mas como o nosso propósito é tratar distribuições periódi cas como funcionais lineares contínuos de $\mathrm{P}_{\mathrm{T}}$ procuraremos usar a notação de $P_{T}^{\prime}$.

Vejamos agora alguns exemplos de distribuições periódicas.

\section{EXEMPLO 1 :}

Seja $g \in L^{1}\left([0, T]^{n}\right)$.

Defina $f: \mathbb{R}^{\mathrm{n}}+\mathbb{C}$ por

$f(x+T m)=g(x), \quad x \in\left[0, T^{n}\right], m \in \mathbb{Z}^{n}$.

Se $\xi \in U_{T}\left(\mathbb{R}^{n}\right)$ e $\theta \in P_{T}\left(\mathbb{R}^{n}\right)$ então f. $\xi \cdot \theta \in L^{1}\left(\mathbb{R}^{n}\right)$ e podemos definir

$$
\begin{aligned}
& L_{f}: P_{T}\left(\mathbb{R}^{n}\right) \rightarrow \mathbb{C} \text { por } \\
& L_{f}(\theta)=\int_{\mathbb{R}^{n}} f \theta \xi(x) d x
\end{aligned}
$$

Vê-se que $L_{f}$ é linear $e$, além do mais, se $\theta_{j} \rightarrow 0$ em $P_{T}\left(\mathbb{R}^{n}\right)$ então $f . \xi \cdot \theta_{j} \rightarrow 0$ quase sempre e

$$
\begin{aligned}
& \left.\mid\left(f \cdot \xi \cdot \theta_{j}\right)(x)\right) \leqq \sup _{x \in \mathbb{R}^{n}}\left|\theta_{j}(x)\right| \cdot|(f . \xi)(x)| \leqq \\
& \leqq C|(f . \xi)(x)| e L^{1}\left(\mathbb{R}^{n}\right)
\end{aligned}
$$

pois como $\theta_{j} \rightarrow 0$ em $P_{T}$ existe $C>0$ tal que

$$
\left|\sup \theta_{j}(x)\right| \leqq C \quad \text { para todo } j \in \mathbb{N} \text {. }
$$


19

Assim, pelo Teorema da Convergência Dominada

$$
\mathrm{I}_{f}\left(\theta_{j}\right)=\int_{\mathbb{R}^{\mathrm{n}}} \mathrm{f} \xi \theta_{j}+0
$$

Portanto, $I_{f} \in P_{T}^{\prime}\left(\mathbb{R}^{n}\right)$.

Além do mais, se $a=\left(a_{1}, \ldots, a_{n}\right) \in \mathbb{R}^{n}$ temos

$$
\begin{aligned}
& \left(L_{f}, \theta\right)=\int_{\mathbb{R}^{n}} f(x) \xi(x) \theta(x) d x= \\
& =\sum_{m \in \mathbb{Z}^{n}} \int_{\mathrm{n}} f(x) \xi(x) \theta(x) d x= \\
& \prod_{i=1}\left[a_{i}+T m_{i}, a_{i}+T m_{i}+T\right] \\
& =\sum_{m \in \mathbb{Z}^{n}} \int_{n} \quad f(y+T m) \xi(y+T m) \theta(y+T m) d y= \\
& \prod_{i=1}\left[a_{i}, a_{i}+T\right] \\
& =\sum_{m \in z^{n}} \int_{n} \quad f(y) \theta(y) \xi(y+T m) d y= \\
& \prod_{i=1}\left[a_{i}, a_{i}+T\right] \\
& =\int_{n} f(y) \theta(y) \sum_{m} e_{\mathbb{Z}^{n}} \xi(y+T m) d y= \\
& \prod_{i=1}\left[a_{i}, a_{i}+T\right] \\
& =\int_{n} f(y) \theta(y) d y \text {. } \\
& \prod_{i=1}\left[a_{i}, a_{i}+T\right]
\end{aligned}
$$

EXEMPLO 2 :

Seja

$$
\begin{aligned}
\delta: \mathbb{P}_{\mathbb{T}}\left(\mathbb{R}^{\mathrm{n}}\right) & \rightarrow \mathbb{C} \\
\theta & \longmapsto \theta(0) .
\end{aligned}
$$


20

Linearidade: Para todas $\theta_{1}, \theta_{2} \in P_{T}\left(\mathbb{R}^{n}\right)$ e $\lambda \in C$ temos:

i) $\delta\left(\theta_{1}+\theta_{2}\right)=\left(\theta_{1}+\theta_{2}\right)(0)=\theta_{1}(0)+\theta_{2}(0)=\delta\left(\theta_{1}\right)+\delta\left(\theta_{2}\right)$;

ii) $\delta\left(\lambda \theta_{1}\right)=\left(\lambda \theta_{1}\right)(0)=\lambda \theta_{1}(0)=\lambda \delta\left(\theta_{1}\right)$.

Continuidade: Se $\theta_{j} \rightarrow 0$ em $P_{T}\left(\mathbb{R}^{n}\right)$ então $\delta\left(\theta_{j}\right)=\theta_{j}(0) \rightarrow 0$ em $\mathbb{C}$. Portanto, $\delta$ é contínua.

Deste modo $\delta \in P_{T}^{\prime}\left(\mathbb{R}^{n}\right)$.

Vejamos qual é o correspondente de $\delta$ em $D_{T}^{\prime}\left(\mathbb{R}^{n}\right)$.

Pelo Teorema 2 sabemos que tal correspondente, digamos $\tilde{\delta}$, é definido por

$$
\langle\tilde{\delta}, \phi\rangle=\left(\delta, \sum_{m \in \mathbb{Z}^{\mathrm{n}}} \phi_{\mathrm{Tm}}\right)=\sum_{\mathrm{m} \in \mathbb{Z}^{\mathrm{n}}{ }^{\phi} \mathrm{Tm}(0)=\sum_{\mathrm{m}} \mathrm{\sum}_{\mathrm{Z}^{\mathrm{n}}} \phi(\mathrm{Tm})} .
$$

$\delta$ é chamada de distribuição $\delta$ de Dirac T-periódica, ou $\delta$ de Dirac concentrada em $\mathrm{Tm}, \mathrm{m} \in \mathbb{Z}^{\mathrm{n}}$.

Vejamos agora como podemos definir em $P_{T}^{\prime}$ algumas opera ções.

Sejam $f, g \in P_{T}^{\prime}\left(\mathbb{R}^{n}\right), \theta, \theta_{1}, \theta_{2} \in P_{T}\left(\mathbb{R}^{n}\right), \lambda \in \mathbb{C} e$ $\theta_{j} \rightarrow 0$ em $\quad P_{T}\left(\mathbb{R}^{n}\right)$.

i) Adição

Defina $\quad f+g: P_{T}\left(\mathbb{R}^{n}\right) \rightarrow \mathbb{C}$

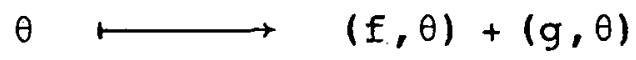

Linearidade: $\quad(f+g)\left(\theta_{1}+\theta_{2}\right)=\left(f, \theta_{1}+\theta_{2}\right)+\left(g, \theta_{1}+\theta_{2}\right)=\left(f, \theta_{1}\right)+$

$+\left(f, \theta_{2}\right)+\left(g, \theta_{1}\right)+\left(g, \theta_{2}\right)=\left(f, \theta_{1}\right)+\left(g, \theta_{1}\right)+\left(f, \theta_{2}\right)+$

$+\left(g, \theta_{2}\right)=(f+g)\left(\theta_{1}\right)+(f+g)\left(\theta_{2}\right)$ 
21

$$
\begin{aligned}
& (f+g)(\lambda \theta)=(f, \lambda \theta)+(g, \lambda \theta)=\lambda(f, \theta)+\lambda(g, \theta)= \\
& =\lambda[(f, \theta)+(g, \theta)]=\lambda(f+g)(\theta) .
\end{aligned}
$$

Continuidade :

$$
(f+g)\left(\theta_{j}\right)=\left(f, \theta_{j}\right)+\left(g, \theta_{j}\right) \rightarrow 0 .
$$

Logo, $f+g \in P_{T}^{\prime}\left(\mathbb{R}^{n}\right)$.

ii) Multiplicação por $\phi \in \mathrm{P}_{\mathrm{T}}\left(\mathbb{R}^{\mathrm{n}}\right)$.

Defina $\quad \phi f: P_{T}\left(\mathbb{R}^{n}\right) \rightarrow \mathbb{C}$

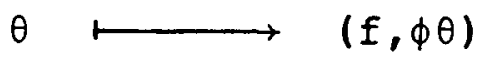

Linearidade:

$$
\begin{aligned}
& (\phi f)\left(\theta_{1}+\theta_{2}\right)=\left(f, \phi\left(\theta_{1}+\theta_{2}\right)\right)=\left(f, \phi \theta_{1}+\phi \theta_{2}\right)= \\
& =\left(f, \phi \theta_{1}\right)+\left(f, \phi \theta_{2}\right)=\phi f\left(\theta_{1}\right)+\phi f\left(\theta_{2}\right) \\
& (\phi f)(\lambda \theta)=(f, \lambda \phi \theta)=\lambda(f, \phi \theta)=\lambda(\phi f)(\theta)
\end{aligned}
$$

Continuidade:

$$
(\phi f)\left(\theta_{j}\right)=\left(f, \phi \theta_{j}\right) \rightarrow 0,
$$

pois para cada $\alpha \in \mathbb{N}^{n}$

$$
\left|D^{\alpha} \phi \theta_{j}(x)\right| \leqq \sup _{|\beta| \leqq|\alpha|}\left|D^{\beta} \phi(x)\right| \quad|\beta| \leqq|\alpha| C_{\beta}\left|D^{\beta} \theta_{j}(x)\right| \rightarrow 0
$$

uniformemente para cada $\alpha$.

Logo, $\phi f \in P_{T}^{\prime}\left(\mathbb{R}^{n}\right)$. 


\section{iii) Translação}

Seja $h$ e $\mathbb{R}^{n}$. Defina $f_{h}: P_{T}\left(\mathbb{R}^{n}\right) \rightarrow \mathbb{C}$

$$
\theta \longmapsto\left(f, \theta_{-h}\right)
$$

onde $\theta_{-h}(x)=\theta(x+h)$.

Linearidade:

$$
\begin{aligned}
& f_{h}\left(\theta_{1}+\theta_{2}\right)=\left(f,\left(\theta_{1}+\theta_{2}\right)_{-h}\right)=\left(f,\left(\theta_{1}\right)_{-h}+\left(\theta_{2}\right)_{-h}\right)= \\
& =\left(f,\left(\theta_{1}\right)_{-h}\right)+\left(f,\left(\theta_{2}\right)_{-h}\right)=\left(f,\left(\theta_{1}\right)_{-h}\right)+ \\
& +\left(f,\left(\theta_{2}\right)_{-h}\right)=f_{h}\left(\theta_{1}\right)+f_{h}\left(\theta_{2}\right) \\
& f_{h}(\lambda \theta)=\left(f,\left(\lambda \theta_{-h}\right)=\left(f, \lambda \theta_{-h}\right)=\lambda\left(f, \theta_{-h}\right)=\lambda f_{h}(\theta)\right.
\end{aligned}
$$

Continuidade:

$$
\begin{aligned}
& f_{h}\left(\theta_{j}\right)=\left(f,\left(\theta_{j}\right)-h\right) \rightarrow 0 \text { em } \mathbb{C} \text { pois para cada } \alpha \in \mathbb{N}^{n} \\
& D^{\alpha}\left(\theta_{j}\right)-h(x)=D^{\alpha} \theta_{j}(x+h) \rightarrow 0 \text { uniformemente. } \\
& \text { Logo, } f_{h} \in P_{T}^{\prime}\left(\mathbb{R}^{n}\right):
\end{aligned}
$$

iv) Reflexão

$$
\text { Defina } \begin{aligned}
\quad \stackrel{\mathrm{f}}{:} \mathrm{P}_{\mathrm{T}}\left(\mathbb{R}^{\mathrm{n}}\right) \longrightarrow \mathbb{C} \\
\theta \longmapsto\left(f, \frac{v}{\theta}\right)
\end{aligned}
$$

onde $\stackrel{v}{\theta}(x)=\theta(-x)$.

Linearidade:

$$
\stackrel{v}{f}\left(\theta_{1}+\theta_{2}\right)=\left(f,\left(\theta_{1}+\theta_{2}\right)^{v}\right)=\left(f, \check{\theta}_{1}+\check{\theta}_{2}\right)=\left(f, \check{\theta}_{1}\right)+\left(f, \check{\theta}_{2}\right)=
$$


23

$$
\begin{aligned}
& =\stackrel{v}{f}\left(\theta_{1}\right)+\stackrel{v}{f}\left(\theta_{2}\right) \\
& \stackrel{v}{f}(\lambda \theta)=\left(\mathbf{f},(\lambda \theta)^{v}\right)=(\tilde{f}, \lambda \stackrel{v}{\theta})=\lambda(\tilde{f}, \stackrel{v}{\theta})=\lambda \stackrel{v}{f}(\theta) .
\end{aligned}
$$

Continuidade:

$$
\begin{aligned}
& \stackrel{v}{f}\left(\theta_{j}\right)=\left(f, \hat{\theta}_{j}\right) \rightarrow 0 \text { em } \mathbb{a} \text { pois, para cada } \alpha \in \mathbb{N}^{n} \\
& \sup \left|D^{\alpha} \theta_{j}(x)\right|=\sup \left|D^{\alpha} \theta_{j}(x)\right|
\end{aligned}
$$

e desse modo $\stackrel{v}{\theta}_{j} \rightarrow 0$ em $P_{T}\left(\mathbb{R}^{n}\right)$ :

Logo, $\stackrel{v}{f} \in P_{T}^{\prime}\left(\mathbb{R}^{n}\right)$.

v) Derivação

Para cada $\alpha \in \mathbb{N}^{\mathrm{n}}$ defina

$$
\begin{aligned}
D^{\alpha} f: P_{T}\left(\mathbb{R}^{n}\right) & \rightarrow \mathbb{C} \\
\theta & \longmapsto\left( \pm,(-1)|\alpha|_{D^{\alpha} \theta}\right)
\end{aligned}
$$

Linearidade:

$$
\begin{aligned}
& D^{\alpha} f\left(\theta_{1}+\theta_{2}\right)=\left(f,(-1)|\alpha|_{D^{\alpha}\left(\theta_{1}+\theta_{2}\right)}\right)= \\
& =\left( \pm,(-1)|\alpha|_{D^{\alpha} \theta_{1}}+(-1)|\alpha|_{D^{\alpha} \theta_{2}}\right)= \\
& =\left(\mathrm{f},(-1)|\alpha|_{D^{\alpha} \theta_{1}}\right)+\left(\mathrm{f},(-1)|\alpha|_{D^{\alpha} \theta_{2}}\right)= \\
& =D^{\alpha} f\left(\theta_{1}\right)+D^{\alpha} f\left(\theta_{2}\right) \text {. }
\end{aligned}
$$

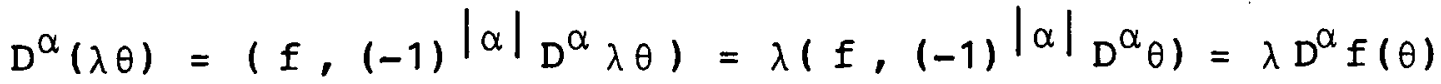

Continuidade:

$$
D^{\alpha} f\left(\theta_{j}\right)=\left( \pm,(-1)|\alpha|_{D^{\alpha} \theta_{j}}\right) \rightarrow 0 \text { em } \mathbb{C} .
$$


Logo, $D^{\alpha} f$ e $P_{T}^{\prime}\left(\mathbb{R}^{n}\right)$.

Notemos que

$$
\begin{aligned}
D^{\alpha}: P_{T}^{\prime}\left(\mathbb{R}^{n}\right) & \longrightarrow P_{T}^{\prime}\left(\mathbb{R}^{n}\right) \\
f & \longmapsto D^{\alpha}
\end{aligned}
$$

é linear e contínuo.

Linearidade: Se $f, g \in P_{T}^{\prime}\left(\mathbb{R}^{n}\right)$ e $\lambda \in \mathbb{C}$ então para toda $\theta \in P_{T}\left(\mathbb{R}^{n}\right)$ temos

$$
\begin{aligned}
& \left(D^{\alpha}(f+g), \theta\right)=\left(f+g,(-1)|\alpha| D^{\alpha} \theta\right)= \\
& =\left(f,(-1)|\alpha| D^{\alpha} \theta\right)+\left(g,(-1)|\alpha| D^{\alpha} \theta\right)= \\
& =\left(D_{f}^{\alpha}, \theta\right)+\left(D^{\alpha} g, \theta\right)=\left(D^{\alpha} f+D^{\alpha} g, \theta\right)
\end{aligned}
$$

e também

$$
\begin{aligned}
& \left(D^{\alpha}(\lambda f), \theta\right)=\left(\lambda f,(-1)|\alpha|_{D^{\alpha} \theta}\right)=
\end{aligned}
$$

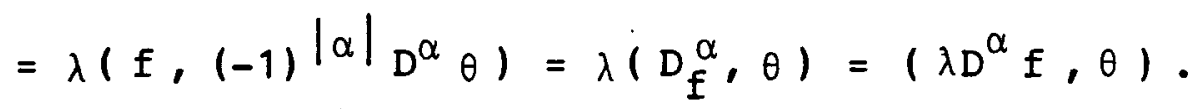

Continuidade: Se $f_{j} \rightarrow 0$ em $P_{T}^{\prime}\left(\mathbb{R}^{n}\right)$ então, para cada $\theta$ e $P_{T}\left(\mathbb{R}^{n}\right)$

$$
\left(D^{\alpha} f_{j}, \theta\right)=\left(f_{j},(-1)|\alpha| D_{\theta}^{\alpha}\right)+0
$$

Notemos ainda que se $f \in P_{T}\left(\mathbb{R}^{n}\right)$ então

$$
\begin{aligned}
& \left(D^{\alpha} f, \theta\right)=\int_{\substack{n \\
j=1}\left[a_{j}, a_{j}+T\right]} D^{\alpha} f(x) \cdot \theta(x) d x= \\
& =(-1)|\alpha| \int_{\prod_{j=1}\left[a_{j}, a_{j}+T\right]} f(x) \cdot D^{\alpha} \theta(x) d x=
\end{aligned}
$$




$$
\begin{aligned}
& =\int_{\mathrm{n}} \quad f(x) \cdot(-1)|\alpha|_{D^{\alpha} \theta(x) d x}=\left(f,(-1)|\alpha|_{\left.D^{\alpha} \theta\right)}\right. \text {. } \\
& \prod_{j=1}\left[a_{j}, a_{j}+T\right]
\end{aligned}
$$

Isto torna mais claro o motivo do fator $(-1)|\alpha|$.

TEOREMA $3 \cdot$ Seja $\phi$ e $C_{c}^{\infty}\left(\mathbb{R}^{n}\right)$ tal que

$$
\int \phi=1, \phi \geqq 0 \text { e } S(\phi) \subset[0, T]^{n}
$$

Seja

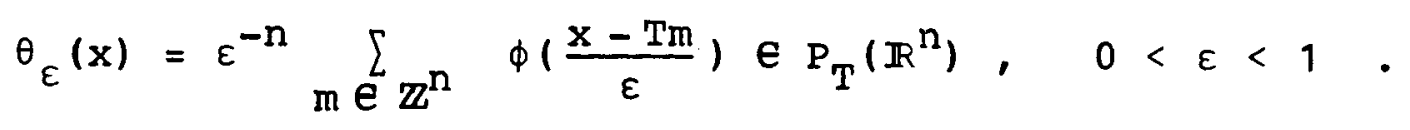

Se f é contínua e T-periódica então

$$
f_{\varepsilon}(x)=\int_{[0, T]^{n}} f(x-y) \theta_{\varepsilon}(y) d y
$$

pertence a $P_{T}\left(\mathbb{R}^{n}\right)$ e $f_{\varepsilon} \rightarrow f$ uniformemente.

\section{DEMONSTRAÇÃO:}

Se $x \in[0, T]^{n}$ então para todo $m=\left(m_{1}, \ldots, m_{n}\right) e z^{n}$, $m \neq 0$ existe $m_{i} \neq 0$ tal que

$$
-\frac{T m_{i}}{\varepsilon} \leqq \frac{x_{i}-T m_{i}}{\varepsilon} \leqq T \frac{\left(1-m_{i}\right)}{\varepsilon}
$$

Se $m_{i} \geqq 1$ então $\frac{x_{i}-T m_{i}}{\varepsilon} \leqq 0$ e, desse modo, $\phi\left(\frac{x-T m}{\varepsilon}\right)=0$.

Se $\quad m_{i} \leqq-1$ então $\quad-\frac{T m_{i}}{\varepsilon} \geqq \frac{T}{\varepsilon} \geqq T \quad e$, portanto, $\phi\left(\frac{x-T m}{\varepsilon}\right)=0$. 
Assim, $\theta_{\varepsilon}(x)=\varepsilon^{-n} \phi\left(\frac{x}{\varepsilon}\right)$, se $x \in[0, T]^{n}$.

Agora,

$$
\begin{aligned}
& f_{\varepsilon}(x)=\int_{[0, T]^{n}} f(x-y) \theta_{\varepsilon}(y) d y=\varepsilon^{-n} \int_{[0, T]^{n}} f(x-y) \phi\left(\frac{y}{\varepsilon}\right) d y= \\
& =\int_{[0, T / \varepsilon]^{n}} f(x-\varepsilon z) \phi(z) d z=\int_{[0, T]^{n}} f(x-\varepsilon z) \phi(z) d z= \\
& =\int_{\mathbb{R}^{n}} f(x-\varepsilon z) \phi(z) d z=\varepsilon^{-n} \int_{\mathbb{R}^{n}} f(u) \phi\left(\frac{x-u}{\varepsilon}\right) d u
\end{aligned}
$$

pois,

$S(\phi) \subset[0, T]^{n} \subset[0, T / \varepsilon]^{n}$

A igualdade

$f_{\varepsilon}(x)=\varepsilon^{-n} \int_{\mathbb{R}^{n}} f(y) \phi\left(\frac{x-y}{\varepsilon}\right) d y$

permiti-nos escrever

onde

$$
f_{\varepsilon}(x)=\varepsilon^{-n} \int_{K_{x}} f(y) \phi\left(\frac{x-y}{\varepsilon}\right) d y
$$

$$
\begin{aligned}
& k_{x}=\left\{y \in \mathbb{R}^{n} ; 0 \leqq \frac{x_{i}-y_{i}}{\varepsilon} \leqq T, i=1, \ldots, n\right\}= \\
& =\left\{y \in \mathbb{R}^{n} ; x_{i}-\varepsilon T \leqq y_{i} \leqq x_{i}, i=1, \ldots, n\right\}
\end{aligned}
$$

$\mathrm{K}_{\mathrm{X}}$ é um compacto.

Dados $x, x^{\prime} \in \mathbb{R}^{n}$, seja $x$ um compacto tal que $K_{X} \cup K_{X^{\prime}} \subset K$. Então existe $C>0$ tal que

$$
\left|f_{\varepsilon}(x)-f_{E}\left(x^{\prime}\right)\right| \leqq \varepsilon^{-n} \int_{\mathbb{R}^{n}}|f(y)|\left|\phi\left(\frac{x-y}{\varepsilon}\right)-\phi\left(\frac{x^{\prime}-y}{\varepsilon}\right)\right| d y . \leqq
$$


27

$$
\begin{aligned}
& \leqq \varepsilon^{-n} \cdot \sup |f(u)| \int_{K}\left|\phi\left(\frac{x-y}{\varepsilon}\right)-\phi\left(\frac{x^{\prime}-y}{\varepsilon}\right)\right| d y \leqq \\
& \leqq \varepsilon^{-n-1} \cdot \int_{K} d y \cdot \sup |f(u)| \cdot\left|x-x^{\prime}\right| .
\end{aligned}
$$

Logo, $f_{\varepsilon}$ é contínua.

Para provar que $f_{\varepsilon}$ é diferenciável qualquer número de vezes, basta derivar sob o sinal de integração a função $\phi$ na expressão de $f_{\varepsilon}$ usada acima e repetir o procedimento acima $\underline{u}$ sando $D^{\alpha} \phi$ ao invés de $\phi$.

Agora,

$$
\begin{aligned}
& \left|f(x)-f_{\varepsilon}(x)\right|=\mid \int_{[0, T]^{n}} f(x) \phi(z) d z- \\
& -\int_{[0, T]^{n}} f(x-\varepsilon z) \phi(z) d z\left|\leqq \int_{[0, T]^{n}}\right| f(x)-f(x-\varepsilon z) \mid \phi(z) d z \leqq \\
& \leqq \sup _{z \in[0, T]^{n}}|f(x)-f(x-\varepsilon z)| .
\end{aligned}
$$

Como f é contínua e periódica, è uniformemente contínua.

Assim, dado $\eta>0$ existe $\delta>0$ tal que

$$
|x-y|<\delta \Rightarrow|f(x)-f(y)|<\varepsilon \text {. }
$$

Como $z \in[0, T]^{n},|z| \leqq \sqrt{n} T$ e para todo $0<\varepsilon<\frac{\delta}{\sqrt{n} T}$ e $x \in \mathbb{R}^{\mathrm{n}}$ temos

$$
\begin{aligned}
& |x-(x-\varepsilon z)|=\varepsilon|z|<\delta \text {. Logo, } \\
& |f(x)-f(x-\varepsilon z)|<n \\
& z e \sup _{[0, T]}|f(x)-f(x-\varepsilon z)|<\eta .
\end{aligned}
$$


Isto prova que $f_{\varepsilon} \rightarrow f$ uniformemente.

TEOREMA 4 . Se $f$ e $g$ são funções continuas e periódicas em $\mathbb{R}^{\mathrm{n}}$ e $\frac{\partial f}{\partial \mathrm{x}_{j}}=g$ no sentido de distribuições periódicas então $f$ é diferenciável em relação a $x_{j}$ e $\frac{\partial f}{\partial x_{j}}=g$ como funções.

\section{DEMONSTRAÇÃO:}

Usando a notação do teorema anterior

$$
\begin{aligned}
& \frac{\partial}{\partial x_{j}} f_{\varepsilon}(x)=\varepsilon^{-n} \int_{[0, T]^{n}} f(y) \frac{\partial}{\partial x_{j}}\left[\phi\left(\frac{x-y}{\varepsilon}\right)\right] d y= \\
& =-\varepsilon^{-n} \int_{[0, T]^{n}} f(y) \frac{\partial}{\partial y_{j}}\left[\phi\left(\frac{x-y}{\varepsilon}\right)\right] d y= \\
& =\varepsilon^{-n} \int_{[0, T]^{n}} g(y) \phi\left(\frac{x-y}{\varepsilon}\right) d y=g_{\varepsilon}(x) .
\end{aligned}
$$

Como $f_{\varepsilon} \rightarrow f$ e $g_{\varepsilon} \rightarrow g$ uniformemente segue que $\frac{\partial f}{\partial x_{j}}=g \cdot$

\section{T- CONVOLUÇÃO}

$\underline{\text { DEFINIÇÃO 1 }}$. Se $f \in P_{T}^{\prime}\left(\mathbb{R}^{n}\right)$ e $\theta \in P_{T}\left(\mathbb{R}^{n}\right)$ defina a convo luçāo de $f$ por $\theta$ como a função

$$
f * \theta: \mathbb{R}^{n} \rightarrow \mathbb{C} \text { dada por }(f * \theta)(a)=\left(f, \hat{\theta}_{a}\right)
$$

onde

$$
\stackrel{v}{\theta}_{a}(x)=\theta(a-x)
$$




\section{EXEMPLO 1 :}

Seja f como no exemplo 1 da seção anterior.

Se $\quad \theta \in P_{T}\left(\mathbb{R}^{n}\right)$ temos,

$$
(f * \theta)(a)=\left(f, v_{a}^{v}\right)=\int_{\prod_{i=1}\left[b_{i}, b_{i}+T\right]} f(x) \theta(a-x) d x
$$

onde substituímos a notação $L_{f}$ do exemplo 1 da seção anterior pela própria $f$.

\section{EXEMPLO 2 :}

$$
\begin{aligned}
& \text { Seja } \theta \in \mathbb{P}_{T}\left(\mathbb{R}^{n}\right) \\
& (\delta * \theta)(a)=\left(\delta, \stackrel{v}{\theta}_{a}\right)=\stackrel{v}{\theta}_{a}(0)=\theta(a) .
\end{aligned}
$$

\section{EXEMPLO 3 :}

$$
\begin{aligned}
& \text { Sejam } \theta \in \mathbb{P}_{T}\left(\mathbb{R}^{n}\right) \text { e } h \in \mathbb{R}^{n} \\
& \left(\delta_{h} \star \theta\right)(a)=\left(\delta_{h}, \check{\theta}_{a}\right)=\left(\delta,\left(\check{\theta}_{a}\right)_{-h}\right)=\left(\check{\theta}_{a}\right)_{-h}(0)= \\
& =\check{\theta}_{a}(h)=\theta(a-h) .
\end{aligned}
$$

\section{EXEMPLO 4 :}

$$
\begin{aligned}
& \text { Sejam } \alpha \in \mathbb{N}^{n} \quad \text { e } \theta \in P_{T}\left(\mathbb{R}^{n}\right) \\
& \left(D^{\alpha} \delta \star \theta\right)(a)=\left(D^{\alpha} \delta, \ddot{\theta}_{a}\right)=\left(\delta,(-1)|\alpha|_{D^{\alpha} \stackrel{v}{\theta}_{a}}\right)=
\end{aligned}
$$

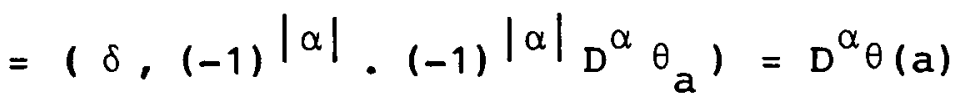

portanto

$$
\mathrm{D}_{\delta}^{\alpha} \star \theta=\mathrm{D}^{\alpha} \theta .
$$


TEOREMA 1. Sejam $f \in P_{T}^{\prime}\left(\mathbb{R}^{n}\right)$ e $\theta \in P_{T}\left(\mathbb{R}^{n}\right)$. Então

$$
f * \theta e P_{T}\left(\mathbb{R}^{n}\right) \quad \text { e } \quad D^{\alpha}(f * \theta)=D^{\alpha} f * \theta=f * D^{\alpha} \theta \text {. }
$$

\section{DEMONSTRACCAO:}

Sejam a $\in \mathbb{R}^{\mathrm{n}}$ e uma seqüência $\left(a_{j}\right)$ tal que $a_{j}+a$.

Para provar a continuidade de $f * \theta$ basta provar que $\theta_{a_{j}} \rightarrow \theta_{a}$ em $P_{T}\left(\mathbb{R}^{n}\right)$, pois dai,

$$
(f * \theta)\left(a_{j}\right)=\left(f, \stackrel{v}{a_{a_{j}}}\right) \rightarrow\left(f, \hat{\theta}_{a}\right)=(f * \theta)(a) .
$$

Mostremos, então, que $\stackrel{v}{\theta_{a_{j}}} \rightarrow \stackrel{v}{\theta_{a}}$ em $P_{T}\left(\mathbb{R}^{n}\right)$.

Dado $\alpha e \mathbb{N}^{n}$, sabemos que, devido às derivadas de $\theta$ se rem limitadas, existe $c>0$ tal que

$$
\left|D^{\alpha} \stackrel{v}{\theta}_{a_{j}}(x)-D^{\alpha} \stackrel{v}{\theta}_{a}(x)\right|=\left|D^{\alpha} \theta\left(a_{j}-x\right)-D^{\alpha} \theta(a-x)\right| \leqq c\left|a_{j}-a\right| .
$$

Como $a_{j} \rightarrow a$, segue-se o resultado.

Seja $e_{i}$ o i-ésimo vetor da base canônica do $\mathbb{R}^{\mathrm{n}}$.

Dada uma seqüência $\left(h_{j}\right), h_{j} \neq 0$ tal que $h_{j} \rightarrow 0$ temos

$$
\begin{aligned}
& {\left[(f * \theta)\left(a+h_{j} e_{i}\right)-(f * \theta)(a)\right] \cdot h_{j}^{-1}=} \\
& =\left[\left(f, v_{a+h_{j} e_{i}}\right)-\left(f, v_{a}\right)\right] \cdot h_{j}^{-1}= \\
& =\left[\left(f_{h_{j} e_{i}}, \check{\theta}_{a}\right)-\left(f, \check{\theta}_{a}\right)\right] \cdot h_{j}^{-1}= \\
& =\left(\left(f_{h_{j} e_{i}}-f\right) \cdot h_{j}^{-1}, \check{\theta}_{a}\right)
\end{aligned}
$$

Mostremos que $g_{j}=\left(f_{h_{j} e_{i}}-f\right) \cdot h_{j}^{-1}$ converge para $\frac{\partial}{\partial x_{i}} f$ em $P_{T}^{\prime}$. 
31

Dada $\phi \in P_{T}\left(\mathbb{R}^{n}\right)$ temos

$$
\begin{aligned}
& \left(g_{j}, \phi\right)=\left(\left(f_{h_{j} e_{i}}-f\right) \cdot h_{j}^{-1}, \phi\right)= \\
& =\left(f,\left(\phi-\phi_{-h_{j} e_{i}}\right) \cdot h_{j}^{-1}\right)
\end{aligned}
$$

Resta, assim, mostrarmos que

$$
\left(\phi-\phi_{-h_{j} e_{i}}\right) \cdot h_{j}^{-1} \rightarrow-\frac{\partial}{\partial x_{i}} \phi \text { em } \quad P_{T}
$$

Para cada $\alpha \in \mathbb{I}^{\mathrm{n}}$ temos

$$
\begin{aligned}
& \left|\partial^{\alpha}\left(\left(\phi-\phi_{-h_{j} e_{i}}\right) h_{j}^{-1}+\frac{\partial}{\partial x_{i}} \phi\right)(x)\right|= \\
& =\left|\left(\partial^{\alpha} \phi(x)-\partial^{\alpha} \phi\left(x+h_{j} e_{i}\right)\right) h_{j}^{-1}+\frac{\partial}{\partial x_{i}} \partial^{\alpha} \phi(x)\right|= \\
& =\left|\frac{\partial}{\partial x_{i}} \partial^{\alpha} \phi(x)-\left(\partial^{\alpha} \phi\left(x+h_{j} e_{i}\right)-\partial^{\alpha} \phi(x)\right) h_{j}^{-1}\right| \rightarrow 0
\end{aligned}
$$

uniformemente, pois $\frac{\partial}{\partial x_{i}} \partial^{\alpha} \phi$ é periódica e contínua, e

$$
\left(\partial^{\alpha} \phi\left(x+h_{j} e_{i}\right)-\partial^{\alpha} \phi(x)\right) h_{j}^{-1}
$$

converge pontualmente para $\frac{\partial}{\partial x_{i}} \partial^{\alpha} \phi(x)$.

Logo, $\frac{\partial}{\partial x_{i}}(f * \theta)=\frac{\partial f}{\partial x_{i}} * \theta ;$ como $\frac{\partial f}{\partial x_{i}} * \theta$ é continua temos que $f * \theta$ e $c^{1}\left(\mathbb{R}^{n}\right)$.

Tambēm,

$$
\begin{aligned}
& \left(\frac{\partial f}{\partial x_{i}} * \theta\right)(a)=\left(\frac{\partial f}{\partial x_{i}}, \vartheta_{a}\right)=\left(f,-\frac{\partial}{\partial x_{i}} \stackrel{v}{a}_{a}\right)= \\
& =\left(f,\left(\frac{\partial \theta}{\partial x_{i}}\right)_{a}^{v}\right)=\left(f * \frac{\partial \theta}{\partial x_{i}}\right)(a)
\end{aligned}
$$

Portanto, 


$$
\frac{\partial}{\partial x_{i}}(f * \theta)=\frac{\partial f}{\partial x_{i}} * \theta=f * \frac{\partial \theta}{\partial x_{i}} .
$$

Usando o princípio da indução vemos que se $\partial^{\alpha}(f \star \theta)=$ $=\partial^{\alpha} f * \theta=f * \partial^{\alpha} \theta$ é contínua então, colocando $\beta=\alpha+e_{i}$ temos que

$$
\partial^{\beta}(f * \theta)=\frac{\partial}{\partial x_{i}} \partial^{\alpha}(f * \theta)=\frac{\partial}{\partial x_{i}}\left(\partial^{\alpha} f * \theta\right)=\partial^{\beta} f * \theta
$$

e, portanto, $\partial^{\beta}(f * \theta)$ também é continua.

Ainda,

$$
\begin{aligned}
& \left(\partial_{f}^{\beta} * \theta\right)(a)=\left(\partial^{\beta} f,{ }_{\theta}^{v}\right)=(-1)|\beta|\left(f, \partial^{\beta_{\theta}^{v}}\right)= \\
& =\left(f,\left(\partial^{\beta} \theta\right)_{a}^{v}\right)=\left(f * \partial^{\beta}\right)(a) .
\end{aligned}
$$

A periodicidade de $f * \theta$ se verifica através de

$$
(f * \theta)(a+T m)=\left(f, \stackrel{v}{\theta_{a}}, T m\right)=\left(f, v_{a}\right)=(f * \theta)(a)
$$

$\underline{\text { TEOREMA 2 }}$. Se $f \in P_{T}^{\prime}\left(\mathbb{R}^{n}\right)$ e $\theta, \rho \in P_{T}\left(\mathbb{R}^{n}\right)$ então

$$
(f * \theta) * \rho=f *(\theta * \rho)
$$

\section{DEMONSTRAÇÃO:}

Seja $a \in \mathbb{R}^{\mathrm{n}}$.

Para cada $k=1,2, \ldots$ tome $\varepsilon=\frac{T}{k}$ e defina

$$
S_{\varepsilon}(x)=\sum_{\substack{1 \leqq m_{i} \leqq k \\ 1 \leqq i \leqq n}} \theta(x-(a+\varepsilon m)) \rho(a+\varepsilon m) \cdot \varepsilon^{n} .
$$

$s_{\varepsilon}$ é a soma de Riemann da função $g(y)=\theta(x-y) \rho(y)$ so bre o cubo $\prod_{j=1}^{n}\left[a_{j}, a_{j}+T\right]$. 
33

Mostremos que $S_{\varepsilon}+\theta * \rho$ em $P_{T}\left(\mathbb{R}^{n}\right)$.

Dado $\alpha \in \mathbb{N}^{\mathrm{n}}$ existem $C>0$ e $M>0$ tais que

$$
\begin{aligned}
& \left|D^{\alpha} \theta(x)\right| \leqq C,|\rho(x)| \leqq C \\
& \left|D^{\alpha} \theta(x)-D^{\alpha} \theta(y)\right| \leqq M|x-y| \quad e \\
& |\rho(x)-\rho(y)| \leqq M|x-y|
\end{aligned}
$$

para todo $x, y \in \mathbb{R}^{n}$.

Defina $\quad B_{\varepsilon, i}=\prod_{j=1}^{n}\left[a_{j}+\varepsilon\left(m_{i}-1\right), a_{j}+\varepsilon m_{i}\right]$

Assim,

$$
\begin{aligned}
& \left|D^{\alpha} S_{\varepsilon}(x)-D^{\alpha}(\theta * \rho)(x)\right|=\mid \underset{1 \leqq m_{i} \leqq k}{\sum_{1 \leqq i \leqq m}} D^{\alpha} \theta(x-(a+\varepsilon m)) \rho(a+\varepsilon m) . \\
& 1 \leqq i \leqq n \\
& \text { - } \varepsilon^{n}-\int_{n} \quad D^{\alpha} \theta(x-y) \rho(y) d y \mid= \\
& \prod_{j=1}\left[a_{j}, a_{j}+T\right] \\
& =1 \sum_{1 \leqq m_{i} \leqq k} \int_{B_{\varepsilon, i}} D^{\alpha} \theta(x-(a+\varepsilon m)) \rho(a+\varepsilon m) d y- \\
& 1 \leqq i \leqq n \\
& -\sum_{1 \leqq m_{i} \leqq k} \int_{B_{\varepsilon, i}} D^{\alpha} \theta(x-y) \rho(y) d y \mid \leqq \\
& 1 \leqq i \leqq n \\
& \leqq \sum_{1 \leqq m_{i} \leqq k} \int_{B_{\varepsilon, i}}\left|D^{\alpha} \theta(x-(a+\varepsilon m)) \rho(a+\varepsilon m)-D^{\alpha} \theta(x-y) \rho(y)\right| d y \leqq \\
& 1 \leqq i \leqq n \\
& \leqq \sum_{1 \leqq m_{i} \leqq k} \int_{B_{\varepsilon, i}}\left(\left|D^{\alpha} \theta(x-(a+\varepsilon m))-D^{\alpha} \theta(x-y)\right||\rho(a+\varepsilon m)|+\right. \\
& 1 \leqq i \leqq n \\
& \left.+\left|D^{\alpha} \theta(x-y)\right||\rho(y)-\rho(a+\varepsilon m)|\right) d y \leqq
\end{aligned}
$$




$$
\begin{aligned}
& \leqq \sum_{\substack{1 \leqq m_{i} \leqq k \\
1 \leqq i \leqq n}} M\left(|\rho(a+\varepsilon m)| \int_{B_{\varepsilon, i}}|y-(a+\varepsilon m)| d y+\right. \\
& \left.+\int_{B_{\varepsilon, i}}\left|D^{\alpha} \theta(x-y)\right||y-(a+\varepsilon m)| d y\right) \leqq \\
& \leqq C M \sum_{\substack{1 \leqq m_{i} \leqq k \\
1 \leqq i \leqq n}}\left(\sqrt{n} \varepsilon \int_{B_{\varepsilon, i}} d y+\sqrt{n} \varepsilon \int_{B_{\varepsilon, i}} d y\right)= \\
& =2 C M \varepsilon^{n+1} \sum_{1 \leqq m_{i} \leqq k} 1=2 C M k^{n} \varepsilon^{n+1}=\frac{2 C M T^{n+1}}{k}+0 \text {. } \\
& 1 \leqq i \leqq n
\end{aligned}
$$

Como $S_{\varepsilon}(x+T m)=S_{\varepsilon}(x)$ temos que $s_{\varepsilon} \rightarrow \theta * \rho$ em $P_{T}\left(\mathbb{R}^{n}\right)$ e, portanto, $\left(S_{\varepsilon}\right)_{x}^{v} \rightarrow(\theta * \rho)_{x}^{v}$. Logo

$$
\begin{aligned}
& (f *(\theta * \rho))(x)=\lim _{\varepsilon \rightarrow 0}\left(f * S_{\varepsilon}\right)(x)= \\
& =\lim _{\substack{\varepsilon \rightarrow 0 \\
\varepsilon^{n}}} \sum_{\substack{1 \leqq m_{i} \leqq k \\
1 \leqq i \leqq n}}(f * \theta)(x-(a+\varepsilon m)) \rho(a+\varepsilon m)= \\
& =\int_{\substack{n \\
j=1}}(f * \theta)(x-y) \rho(y) d y=((f * \theta) * \rho)(x) .
\end{aligned}
$$

TEOREMA 3 $\cdot$ Seja $F: P_{T}\left(\mathbb{R}^{n}\right) \rightarrow P_{T}\left(\mathbb{R}^{n}\right)$ um operador linear con tínuo tal que para todo $h \in \mathbb{R}^{\mathrm{n}}$ e toda $\theta \in \mathrm{P}_{\mathrm{T}}\left(\mathbb{R}^{\mathrm{n}}\right)$

$$
\left[T_{h} F(\theta)\right](x)=F(\theta)(x-h)=F\left(\theta_{h}\right)(x)=F\left(T_{h} \theta\right)(x)
$$

Então existe uma única f $e P_{T}^{\prime}\left(\mathbb{R}^{n}\right)$ tal que $F(\theta)=f \star \theta$ para toda $\theta \in \mathrm{P}_{\mathrm{T}}\left(\mathbb{R}^{\mathrm{n}}\right)$. 
DEMONSTRAÇ尺̃O:

Defina $\quad \mathrm{f}: \mathrm{P}_{\mathrm{T}}\left(\mathbb{R}^{\mathrm{n}}\right) \rightarrow \mathbb{C}$

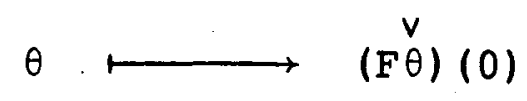

A linearidade de $f$ é imediata.

Se $\theta_{j} \rightarrow 0$ em $P_{T}\left(\mathbb{R}^{n}\right)$ então, por continuidade de $F$, $\stackrel{v}{F \theta_{j}} \rightarrow 0$ em $P_{T}\left(\mathbb{R}^{n}\right)$ e, conseqüentemente, $\left(F^{v}\right)(0) \rightarrow 0$ em a.

Logo $f \in P_{T}^{\prime}\left(\mathbb{R}^{n}\right)$.

Para todo $h \in \mathbb{R}^{\mathrm{n}}$.

$(F \theta)(h)=\left(T_{-h}(F \theta)\right)(0)=\left(\left(T_{-h} F\right) \theta\right)(0)=\left(F\left(T_{-h} \theta\right)\right)(0)=$

$=\left(f,\left(T_{-h} \theta\right)^{v}\right)=\left(f, \ddot{\theta}_{h}\right)=(f * \theta)(h)$

pois, $\quad\left(T_{-h} \theta\right)^{v}(x)=\left(T_{-h} \theta\right)(-x)=\theta(-x+h)=\stackrel{v}{\theta}_{h}(x)$.

Unicidade: Sejam $f, g \in P_{T}^{\prime}\left(\mathbb{R}^{n}\right)$ tais que $F(\theta)=f \star \theta=g * \theta$ para toda $\theta \in P_{T}\left(\mathbb{R}^{n}\right)$.

Então,

$(f, \theta)=(f * \stackrel{v}{\theta})(0)=(g * \stackrel{v}{\theta})(0)=(g, \theta)$.

Logo, $f=g$.

DEFINIÇÃO 2. Sejam $f, g \in P_{T}^{\prime}\left(\mathbb{R}^{n}\right)$.

Definimos $h=f * g$ como sendo a única distribuição peri ódica tal que

$E *(g * \theta)=h * \theta, \quad \theta \in P_{T}\left(\mathbb{R}^{n}\right)$.

h é chamada de T-convolução de $f$ por $g$. 
36

Para verificar que a definição é possivel, mostremos que

$$
\begin{aligned}
F: P_{T}\left(\mathbb{R}^{n}\right) & \longrightarrow P_{T}\left(\mathbb{R}^{n}\right) \\
\theta & \longmapsto f *(g * \theta)
\end{aligned}
$$

é linear-continuo e comuta com as translações $\mathrm{T}_{\mathrm{h}}$.

Linearidade: Se $\theta, \rho \in P_{T}\left(\mathbb{R}^{n}\right)$ e $\lambda \in \mathbb{C}$ então

i)

$$
\begin{aligned}
& F(\theta+\rho)=f *(g *(\theta+\rho))=f *(g * \theta+g * \rho)= \\
& =f *(g * \theta)+f *(g * \rho)=F(\theta)+F(\rho) .
\end{aligned}
$$

ii)

$$
\begin{aligned}
& F(\lambda \theta)=f *(g *(\lambda \theta))=f *(\lambda(g * \theta))= \\
& =\lambda f *(g * \theta)=\lambda F(\theta) .
\end{aligned}
$$

Continuidade: Seja $\theta_{j} \rightarrow 0$ em $P_{T}\left(\mathbb{R}^{n}\right)$.

Dado $a \in \mathbb{N}^{\mathrm{n}}$ existem $\mathrm{C}_{1}, \mathrm{C}_{2}>0 \quad \mathrm{~m}_{1}, \mathrm{~m}_{2}$ e $\mathbb{N}$ tais que

$$
\begin{aligned}
& \left|D^{\alpha}\left(F\left(\theta_{j}\right)\right)(a)\right|=\left|f * D^{\alpha}\left(g * \theta_{j}\right)(a)\right|= \\
& =\left|\left(f,\left(D^{\alpha}\left(g * \theta_{j}\right)\right)_{a}^{v}\right)\right| \leqq C_{1} \sum_{|\beta| \leqq m_{1}} \sup _{x}\left|D^{\beta}\left(D^{\alpha}\left(g * \theta_{j}\right)\right)_{a}^{v}\right|= \\
& =C_{1} \sum_{|\beta| \leqq m_{1}} \sup _{x}\left|\left(D^{\beta+\alpha}\left(g * \theta_{j}\right)\right)_{a}^{v}\right|= \\
& =c_{1} \sum_{|\beta| \leqq m_{1}} \sup _{x}\left|\left(g * D^{\beta+\alpha} \theta_{j}\right)(a-x)\right|= \\
& =C_{1} \sum_{|\beta| \leqq m_{1}} \sup _{x}\left|\left(g,\left(D^{\beta+\alpha} \theta_{j}\right)_{a-x}^{v}\right)\right| \leqq \\
& \leqq C_{1} C_{2} \sum_{|\beta| \leqq m_{1}} \sup _{x}\left|\sum_{|\gamma| \leqq m_{2}} \sup _{y}\right| D^{\gamma}\left(D^{\beta+\alpha} \theta_{j}\right)_{a-x}^{v}||= \\
& =c_{1} C_{2} \underset{|\beta| \leqq m_{1}}{\sum_{x}} \sup _{x} \sum_{\gamma \mid \leqq m_{2}} \sup _{y} D^{\gamma+\beta+\alpha} \theta_{j}(a-x-y)||=
\end{aligned}
$$




$$
=C_{1} C_{2} \underset{|\beta| \leq m_{1}}{\sum_{x}} \sup _{|\gamma| \leq m_{2}} \sup _{z} D^{\gamma+\beta+\alpha} \theta_{j}(z-x) \mid \rightarrow 0
$$

uniformemente.

$$
\text { Portanto, F é contínuo. }
$$

Comutatividade: Sejam $h \in \mathbb{R}^{\mathrm{n}}$ e $\theta \in \mathrm{P}_{\mathrm{T}}\left(\mathbb{R}^{\mathrm{n}}\right)$

$$
\begin{aligned}
& T_{h}(F \theta)(a)=T_{h}(f *(g * \theta))(a)=(f *(g * \theta))(a-h)= \\
& =\left(f,(g * \theta)_{a-h}^{v}\right)=\left(f,\left(T_{h}(g * \theta)\right)_{a}^{v}\right)= \\
& =\left(f * T_{h}(g * \theta)\right)(a)=\left(f *\left(g * T_{h} \theta\right)\right)(a)=F\left(T_{h} \theta\right)(a) . \\
& \text { Justificativa da igualdade } T_{h}(g * \theta)=g * T_{h}(\theta): \\
& T_{h}(g * \theta)(a)=g * \theta(a-h)=(g, \stackrel{v}{a-h})= \\
& =\left(g,\left(T_{h} \theta\right)_{a}^{v}\right)=\left(g * T_{h} \theta\right)(a) .
\end{aligned}
$$

Antes de mostrarmos algumas propriedades da T-convolução vejamos o seguinte lema:

LEMA 1. Seja $\phi \in C_{C}^{\infty}\left(\mathbb{R}^{n}\right)$ tal que

$$
\int_{\mathbb{R}^{\mathrm{n}}} \phi=1, \phi \geqq 0 \quad \text { e } \quad S(\phi) \subset[0, \mathrm{~T}]^{\mathrm{n}} .
$$$$
\text { Seja } \theta_{\varepsilon}(x)=\varepsilon^{-n} \sum_{m} e_{\mathbb{Z}^{n}} \phi\left(\frac{x-T m}{\varepsilon}\right) \text { onde } 0<\varepsilon<1 \text {. Ob }
$$
serve que ${ }_{\varepsilon}$ e $\mathrm{P}_{\mathrm{T}}\left(\mathbb{R}^{\mathrm{n}}\right)$. Então, se $\psi \in \mathrm{P}_{\mathrm{T}}\left(\mathbb{R}^{\mathrm{n}}\right), \psi * \theta_{\varepsilon} \rightarrow \psi$ em $P_{T}\left(\mathbb{R}^{n}\right)$.

DEMONSTRAÇÃO:

Seja $\alpha \in \mathbb{N}^{\mathrm{n}}$. 
38

$$
\begin{aligned}
& D^{\alpha}\left(\psi * \theta_{\varepsilon}\right)(a)=\int_{[0, T]^{n}} D^{\alpha} \psi(a-x) \theta_{\varepsilon}(x) d x= \\
& =\varepsilon^{-n} \int_{[0, T]^{n}} D^{\alpha} \psi(a-x) \phi\left(\frac{x}{\varepsilon}\right) d x
\end{aligned}
$$

pois, se $x \in[0, T]^{n}$ então para todo $m=\left(m_{1}, \ldots, m_{n}\right) e \mathbb{Z}^{n}$, $m \neq 0$ existe $m_{i} \neq 0$ tal que

$$
-\frac{T m_{i}}{\varepsilon} \leqq \frac{x_{i}-T m_{i}}{\varepsilon} \leqq \frac{T\left(1-m_{i}\right)}{\varepsilon}
$$

Se $m_{i} \geqq 1$ então $\frac{x_{i}-T m_{i}}{\varepsilon} \leqq 0$ e então $\phi\left(\frac{x-T m}{\varepsilon}\right)=0$.

Se $m_{i} \leqq-1$ então $-\frac{T m_{i}}{\varepsilon} \geqq \frac{T}{\varepsilon} \geqq T$ e então

$\phi\left(\frac{x-T m}{\varepsilon}\right)=0$

Deste modo $\theta_{\varepsilon}(x)=\varepsilon^{-n_{\phi}\left(\frac{x}{\varepsilon}\right)}$ se $x \in[0, T]^{n}$.

Também,

$$
\begin{aligned}
& \varepsilon^{-n} \int_{[0, T]^{n}} D^{\alpha} \psi(a-x) \phi\left(\frac{x}{\varepsilon}\right) d x=\int_{[0, T / \varepsilon]^{n}} D^{\alpha} \psi(a-\varepsilon y) \phi(y) d y= \\
& =\int_{[0, T]^{n}} D^{\alpha} \psi(a-\varepsilon y) \phi(y) d y
\end{aligned}
$$

pois

$$
S(\phi) \subset[0, T]^{\mathrm{n}} \subset\left[0, \frac{\mathrm{T}}{\varepsilon}\right]^{\mathrm{n}}
$$

Assim,

$$
\begin{aligned}
& \left|D^{\alpha} \psi(a)-\left(D^{\alpha} \psi \star \theta_{\varepsilon}\right)(a)\right|=1 \int_{[0, T]^{n}}\left(D^{\alpha} \psi(a)-\right. \\
& \left.-D^{\alpha} \psi(a-\varepsilon x)\right) \phi(x) d x\left|\leqq \int_{[0, T]^{n}} \phi(x) d x \cdot \sup \right| D^{\alpha} \psi(a)- \\
& -D^{\alpha} \psi(a-\varepsilon x)\left|\leqq C \cdot \sup _{x \in[0, T]^{n}}\right| \varepsilon x \mid=\sqrt{n} C T \cdot \varepsilon .
\end{aligned}
$$


39

Logo, $\psi * \theta_{\varepsilon}+\psi$ em $\mathrm{P}_{\mathrm{T}}\left(\mathbb{R}^{\mathrm{n}}\right)$

$\underline{\text { LEMA 2 }}$. Sejam $f, g \in P_{T}^{\prime}\left(\mathbb{R}^{n}\right)$. Então, $f=g$ se, e somente se, $f * \theta=g * \theta$ para toda $\theta \in P_{T}\left(\mathbb{R}^{n}\right)$.

DEMONSTRAÇÃO:

$$
\begin{aligned}
& \text { Se } f=g \text { então } \\
& (f * \theta)(a)=\left(f, v_{a}\right)=\left(g, \vee_{a}\right)=(g * \theta)(a) . \\
& \text { Se } g * \theta=f * \theta \text { então } \\
& (f, \theta)=(f * \stackrel{v}{\theta})(0)=(g * \stackrel{v}{\theta})(0)=(g, \theta) .
\end{aligned}
$$

TEOREMA 4. Se $f, g, h$ e $P_{T}^{\prime}\left(\mathbb{R}^{n}\right)$ então

i) $(f * g) * h=f *(g * h)$

ii) $f * g=g * f$

DEMONSTRAÇÃO:

Seja $\quad \theta \in P_{T}\left(\mathbb{R}^{n}\right)$.

i)

$$
\begin{aligned}
& ((f * g) * h) * \theta=(f * g) *(h * \theta)=f *(g *(h * \theta))= \\
& =f *((g * h) * \theta)=(f *(g * h)) * \theta .
\end{aligned}
$$

Usando o Jjema 2 concluimos que

$$
(f \star g) * h=f *(g * h)
$$

ii) Seja $\theta_{\varepsilon}$ como no Lema 1 .

Provemos primeiramente que $\theta_{\varepsilon} \star \theta=\theta * \theta_{\varepsilon}$. 


$$
\begin{aligned}
& \left(\theta_{\varepsilon} \star \theta\right)(a)=\int_{[0, T]^{n}} \theta_{\varepsilon}(a-x) \theta(x) d x= \\
& =\int_{n} \quad \theta_{\varepsilon}(y) \theta(a-y) d y= \\
& \prod_{j=1}\left[a_{j}, a_{j}+T\right] \\
& =\int_{n} \theta(a-y) \theta_{\varepsilon}(y) d y=\left(\theta * \theta_{\varepsilon}\right)(a) \text {. } \\
& \prod_{j=1}\left[a_{j}, a_{j}+T\right]
\end{aligned}
$$

Agora,

$$
\begin{aligned}
& (f * g) *\left(\theta * \theta_{\varepsilon}\right)=f *\left(g *\left(\theta * \theta_{\varepsilon}\right)\right)=f *\left((g * \theta) * \theta_{\varepsilon}\right)= \\
& =f *\left(\theta_{\varepsilon} *(g * \theta)\right)=\left(f * \theta_{\varepsilon}\right) *(g * \theta) .
\end{aligned}
$$

Por outro lado,

$$
\begin{aligned}
& (g * f) *\left(\theta * \theta_{\varepsilon}\right)=(g * f) *\left(\theta_{\varepsilon} * \theta\right)=g *\left(f *\left(\theta_{\varepsilon} * \theta\right)\right)= \\
& =g *\left(\left(f * \theta_{\varepsilon}\right) * \theta\right)=g *\left(\theta *\left(f * \theta_{\varepsilon}\right)\right)= \\
& =(g * \theta) *\left(f * \theta_{\varepsilon}\right)=\left(f * \theta_{\varepsilon}\right) *(g * \theta) .
\end{aligned}
$$

Comparando,

$$
(f * g) *\left(\theta * \theta_{\varepsilon}\right)=(g * f) *\left(\theta * \theta_{\varepsilon}\right)
$$

Quando $\varepsilon \rightarrow 0$, pelo Lema $1, \theta * \theta_{\varepsilon} \rightarrow \theta$ em $\mathrm{P}_{\mathrm{T}}$. Logo $(f * g) * \theta=(g * f) * \theta$.

Pelo Lema $2, f * g=g * f$.

$\underline{T E O R E M A} 5 \cdot$ Sejam $f, g \in P_{T}^{\prime}\left(\mathbb{R}^{n}\right)$ e a $e \mathbb{R}^{n}$. 
41

i) $(f * g)_{a}=f_{a} * g=f * g_{a}$

ii) $D^{\alpha}(f * g)=D^{\alpha} f * g=f * D^{\alpha} g, \forall \alpha e \mathbb{N}^{n}$.

Mostremos primeiramente que se $\theta$ e $P_{T}$ então $f_{a} * \theta=$ $=\mathbf{f} * \theta_{-a} \cdot$

Observe que isto não contradiz il, pois, se Le é o re presentante da função $\theta$ como distribuição periódica então,

$$
\begin{aligned}
& L_{-a}=(L \theta)_{a} \text { De fato, } \\
& \left(f_{a} * \theta\right)(x)=\left(f_{a}, v_{x}^{v}\right)=\left(f,\left(v_{x}\right)_{-a}\right)=\left(f, v_{x+a}\right)= \\
& =\left(f,\left(\theta_{-a}\right)_{x}^{v}\right)=\left(f * \theta_{-a}\right)(x) . \\
& \text { Assim, } \\
& (f * g)_{a} * \theta=(f * g) * \theta_{-a}=f *\left(g * \theta_{-a}\right)= \\
& =f *\left(g_{a} * \theta\right)=\left(f * g_{a}\right) * \theta .
\end{aligned}
$$

Portanto,

$$
(f * g)_{a}=f * g_{a} .
$$

Também,

$$
\begin{aligned}
& (f * g)_{a} * \theta=(g * f)_{a} * \theta=(g * f) * \theta_{-a}=g *\left(f * \theta_{-a}\right)= \\
& =g *\left(f_{a} * \theta\right)=\left(g * f_{a}\right) * \theta=\left(f_{a} * g\right) * \theta . \\
& \text { Mostremos agora } i i) \\
& \left.\left(D^{\alpha}(f * g) \star \theta\right)(a)=\left(D^{\alpha}(f * g), \check{\theta}_{a}\right)=\left(f * g,\left.(-1)^{\alpha}\right|_{D^{\alpha}\left(\theta_{a}\right.}\right)\right)= \\
& =\left(f * g,\left(D^{\alpha} \theta\right)_{a}^{v}\right)=\left((f * g) * D^{\alpha} \theta\right)(a)=\left(f *\left(g * D^{\alpha} \theta\right)\right)(a)=
\end{aligned}
$$




$$
\begin{aligned}
& =\left(f *\left(g,\left(D^{\alpha} \theta\right)_{x}^{v}\right)\right)(a)=(-1)|\alpha|\left(f *\left(g, D^{\alpha}\left(v_{x}^{v}\right)\right)\right)(a)= \\
& =\left(f *\left(D^{\alpha} g, v_{x}\right)\right)(a)=\left(f *\left(D_{g}^{\alpha} * \theta\right)\right)(a)= \\
& =\left(\left(f * D^{\alpha} g\right) * \theta\right)(a) .
\end{aligned}
$$

Portanto,

$$
D^{\alpha}(f * g)=f * D^{\alpha} g
$$

Por comutatividade obtém-se a outra igualdade.

\section{EXEMPLO 5 :}

$$
\begin{aligned}
& \text { Sejam } f \in P_{T}^{\prime}\left(\mathbb{R}^{n}\right) \text { e } \theta e P_{T}\left(\mathbb{R}^{n}\right) . \\
& (f * \delta, \theta)=(f * \delta * \stackrel{v}{\theta})(0)=(f * \stackrel{v}{\theta})(0)=(f, \theta) . \\
& \text { Logo } f * \delta=f \text {. }
\end{aligned}
$$

EXEMPLO 6 :

Sejam $\alpha e \mathbb{N}^{n}$ e $f e P_{T}^{\prime}\left(\mathbb{R}^{n}\right)$.

$$
D^{\alpha} f=D^{\alpha}(f * \delta)=D^{\alpha} f * \delta=f * D^{\alpha} \delta \text {. }
$$

\section{EXEMPLO 7 :}

Seja $P(D)=\sum_{|\alpha| \leqq m} C_{\alpha} D^{\alpha}$ um operador diferencial linear parcial a coeficientes constantes. Se $f \in P_{T}^{\prime}\left(\mathbb{R}^{n}\right)$ então

$$
\begin{aligned}
& P(D) f=\sum_{|\alpha| \leqq m} c_{\alpha} D^{\alpha} f=\mid \alpha \sum_{\leqq m} c_{\alpha} f * D^{\alpha} \delta= \\
& \left.=\sum_{|\alpha| \leqq m} f *\left(c_{\alpha} D^{\alpha} \delta\right)=f * 1 \mid \sum_{|\alpha| \leqq m} c_{\alpha} D^{\alpha} \delta\right)=f *(P(D) \delta) .
\end{aligned}
$$


Assim, toda equação diferencial parcial linear com coefi cientes constantes

$$
P(D) u=f \text {, onde } u, f \in P_{T}^{\prime}\left(\mathbb{R}^{n}\right)
$$

pode ser expressa como uma equação da T-convolução

$$
u * u_{0}=f \quad,
$$

onde

$$
u_{0}=P(D) \delta
$$

\section{EXEMPLO 8 :}

Uma equação linear de diferenças com coeficientes constan tes pode ser expressa como uma equação da T-convolução.

Vejamos

$$
\begin{aligned}
& \sum_{|\alpha| \leqq m} c_{\alpha} f_{\mathrm{T}_{\alpha}}=\sum_{|\alpha| \leq m} c_{\alpha}(f * \delta)_{\mathrm{T}_{\alpha}}=\sum_{|\alpha| \leqq m} c_{\alpha} f * \delta_{\mathrm{T}_{\alpha}}= \\
& =\mathrm{f} *\left(\sum_{|\alpha|^{\prime} \leqq \mathrm{m}} \mathrm{c}_{\alpha} \delta_{\mathrm{T}_{\alpha}}\right) .
\end{aligned}
$$

$\underline{\text { TEOREMA } 6} \cdot$ Seja $C: P_{T}^{\prime}\left(\mathbb{R}^{n}\right) \times P_{T}^{\prime}\left(\mathbb{R}^{n}\right) \rightarrow P_{T}^{\prime}\left(\mathbb{R}^{n}\right)$

$$
\begin{aligned}
&(f, g) \longrightarrow f * g \\
& C \text { é sequiencialmente contínuo em cada variável. }
\end{aligned}
$$

DEMONSTRAÇÃO:

Como C é simétrico, basta demonstrar em uma variável.

Como C é linear, basta verificar que se $\mathrm{f}_{j} \rightarrow 0$ em $\mathrm{P}_{\mathrm{T}}^{\prime}$ então 
$C\left(f_{j}, g\right)=f_{j} * g \rightarrow 0$ em $\quad P_{T}^{\prime}$

Seja $\theta$ e $P_{T}\left(\mathbb{R}^{n}\right)$.

$\left(C\left(f_{j}, g\right), \theta\right)=\left(f_{j} * g, \theta\right)=\left(f_{j} *(g * \theta)\right)(0)$

Como $g * \stackrel{v}{\theta}$ e $P_{T}$ e $f_{j} \rightarrow 0$ em $P_{T}^{\prime}$ segue-se que

$\left(f_{j} *(g * \stackrel{v}{\theta})\right)(0)=\left(f_{j},(g * \stackrel{v}{\theta})^{v}\right) \rightarrow 0$.

4. A ALGEBRA $\left(\mathrm{P}_{\mathrm{T}}^{\prime}, *,+\right)$

o espaço vetorial $P_{T}^{\prime}\left(\mathbb{R}^{n}\right)$ munido das operações T-convo lução e adição possui uma estrutura de álgebra comutativa com $\underline{\text { e }}$ lemento neutro $\delta$ em relação à T-convolução. Designaremos esta álgebra por álgebra da T-convolução.

o próximo teorema nos dará uma condição necessāria e sufí ciente para que possamos encontrar u em $\mathrm{P}_{\mathrm{T}}^{\prime}$ tal que

$$
f * u=g,
$$

onde $f$ e $g$ são distribuições periódicas conhecidas anterior mente.

Antes disso, definamos o inverso (segundo a T-convolução) de um elemento de $P_{T}^{\prime}$.

DEFINIÇÃo 1. f e $\mathrm{P}_{\mathrm{T}}^{\prime}$ é dito inversivel se existir $g$ e $\mathrm{P}_{\mathrm{T}}^{\prime}$ tal que

$$
f * g=g * f=\delta \text {. }
$$


Uma $g$ deste tipo é dita inversa de $f$.

TEOREMA 1. Se existir inversa de uma distribuição periódica ela é única.

DEMONSTRAÇÃO:

Sejam $g$ e $h$ inversas de $f$ então, $g=g * \delta=g *(f * h)=(g * f) * h=\delta * h=h$.

Denotemos por $f^{-1}$ o inverso de $f$, quando existir.

Nem todos os elementos de $P_{T}^{\prime}$ possuem inverso, como é o caso de qualquer $\theta \in \mathrm{P}_{\mathrm{T}}$. Pois, como visto no Teorema 1 da se cão anterior, $\mathbf{f} * \theta$ e $\mathrm{P}_{\mathrm{T}}$ para toda $\mathbf{f}$ e $\mathrm{P}_{\mathrm{T}}^{\prime}$. Logo, é impossi vel encontrar $f \in \mathrm{P}_{\mathrm{T}}^{\prime}$ tal que $f * \theta=\delta$, já que $\delta \notin \mathrm{P}_{\mathrm{T}}$, pois, se assim fosse teríamos

$$
\begin{aligned}
& \frac{\partial}{\partial x_{j}} \delta(x)=\lim _{h \rightarrow 0}\left(\delta\left(x+h e_{j}\right)-\delta(x)\right) h^{-1}= \\
& =\lim _{h \rightarrow 0}\left(h^{-1}\left(\delta_{x+h e_{j}}-\delta_{x}, \delta\right)\right)= \\
& =\lim _{h \rightarrow 0}\left(h^{-1}\left(\left(\delta_{x}\right)_{h e_{j}}-\delta_{x}\right), \delta\right) \rightarrow\left(\frac{\partial}{\partial x_{j}} \delta_{x}, \delta\right)= \\
& =\left(\delta_{x},-\frac{\partial}{\partial x_{j}} \delta\right)=-\frac{\partial}{\partial x_{j}} \delta(x) . \\
& \text { Logo, } \frac{\partial}{\partial x_{j}} \delta(x)=0, i . e ́ ., \delta \text { é constante. } \\
& \text { Como }\left(\delta, e^{i w x}\right)=1 \text { e }(\delta, 0)=0 \text { vemos que a suposição }
\end{aligned}
$$
foi absurda. 
$\underline{\text { TEOREMA } 2}$. Seja $f$ e $P_{T}^{\prime}\left(\mathbb{R}^{n}\right)$.

Uma condição necessária e suficiente para que a equação

$$
f * u=g
$$

tenha, para cada $g \in P_{T}^{\prime}\left(\mathbb{R}^{n}\right)$, uma solução em $P_{T}^{\prime}\left(\mathbb{R}^{n}\right)$ é que e xista $\mathrm{f}^{-1}$.

No caso afirmativo a solução é ūnica e dada por $f^{-1} * g$.

DEMONSTRAČAO:

Necessidade: Existe u e $P_{T}^{\prime}\left(\mathbb{R}^{n}\right)$ tal que

$$
f * u=\delta \quad \text { Logo, } u=f^{-1} \text {. }
$$

Suficiência: Suponha que exista $\mathrm{f}^{-1}$.

$$
\begin{aligned}
& \text { Tome } u=f^{-1} * g \text { Então, } \\
& f * u=f *\left(f^{-1} * g\right)=\left(f * f^{-1}\right) * g=\delta * g=g . \\
& \text { Se } f * v=g \text { então } \\
& f^{-1} *(f * v)=f^{-1} * g \\
& \left(f^{-1} * f\right) * v=f^{-1} * g \\
& v=f^{-1} * g
\end{aligned}
$$

E importante observar que o teorema diz respeito à exis tência e unicidade de solução da equação de $T$-convolução $f * u=g$ quando a resolvemos para toda $g \in P_{T}^{\prime}\left(\mathbb{R}^{n}\right)$.

Vejamos o seguinte exemplo: 


\section{EXEMPLO 1 :}

Seja $f=1$ e $u_{n}(t)=e^{i w n t}$ onde $w=\frac{2 \pi}{T}, n \in \mathbb{Z}^{\star}$ e $t e \mathbb{R}$.

\section{Então,}

$$
\left(f * u_{n}\right)\left(t==\int_{a}^{a+T} f(t-s) u_{n}(s) d s=\int_{a}^{a+T} e^{i w n s} d s=0 .\right.
$$

Assim, a equação $f * u=0$ possui infinitas soluções.

\section{A SERIE DE FOURIER}

DEFINIÇÃO 1 $\cdot$ Seja $\left(f_{m}\right)_{m} \in \mathbb{Z}^{k}$ uma seqüēncia em $P_{T}^{\prime}\left(\mathbb{R}^{n}\right)$. Dizemos que a série $\sum_{m e \mathbb{Z}^{k}} \mathrm{f}_{m}$ é convergente em $P_{T}^{\prime}\left(\mathbb{R}^{n}\right)$ se a seqüência $s_{\ell}=\sum_{|m| \leqq \ell} f_{m}$ for convergente em $P_{T}^{\prime}\left(\mathbb{R}^{n}\right)$.

TEOREMA 1. Se $f=\sum_{m \in \mathbb{Z}^{k}} f_{m} e P_{T}^{\prime}\left(\mathbb{R}^{I}\right)$ então $D^{\alpha} f=\sum_{m} e_{\mathbb{Z}^{k}} D^{\alpha} f_{m}$. DEMONSTRAÇÃO:

Dados $\theta \in \mathrm{P}_{\mathrm{T}}\left(\mathbb{R}^{\mathrm{n}}\right)$ e $\varepsilon>0$ existe $\ell_{0} \in \mathbb{N}$ tal que para todo $l \geqq l_{0}$ tem-se

$$
\left|\left(f-\sum_{|m| \leqq \ell} f_{m}, D^{\alpha} \theta\right)\right|<\varepsilon .
$$

Deste modo, 


$$
\begin{aligned}
& \left|\left(D^{\alpha} f-\sum_{|m| \leqq \ell} D^{\alpha} f_{m}, \theta\right)\right|=\left|\left(D^{\alpha}\left(f-\sum_{|m| \leqq l} f_{m}\right), \theta\right)\right|= \\
& =\left|\left(f-\sum_{|m| \leqq l} f_{m}, D^{\alpha} \theta\right)\right|<\varepsilon .
\end{aligned}
$$

As definições a seguir serão úteis para caracterizarmos os elementos de $\mathrm{P}_{\mathrm{T}}$ e $\mathrm{P}_{\mathrm{T}}^{\prime}$.

DEFINIÇÃO 2 - Uma seqüência numérica é dita rapidamente decres cente se para cada $k \in \mathbb{Z}^{+}$existir $c>0$ tal que:

$$
\left|c_{m}\right| \leqq \frac{c}{|m|^{k}} \quad \text { para todo } m \in \mathbb{z}^{n}, m \neq 0
$$

Equivalentemente, $\left(c_{m}\right)$ é rapidamente decrescente se para cada $k \in \mathbb{Z}^{+}$existir $c>0$ tal que $\left|c_{m}\right| \leqq \frac{c}{(1+|m|)^{k}}$ para todo $m \in \mathbb{Z}^{n}$. Pois, se para cada $k \in \mathbb{Z}^{+}$existir $c>0$ tal que

$$
\left|c_{m}\right| \leqq \frac{c}{|m|^{k}}, m \neq 0, m \in \mathbb{Z}^{n}
$$

então, se $m \neq 0,1+|m| \leqq 2|m|$ e

$$
\left|c_{m}\right| \leqq \frac{c}{|m|^{k}} \leqq \frac{2^{k} c}{(1+|m|)^{k}}
$$

Colocando $M=\max \left\{2^{\mathrm{k}} \mathrm{C},|\mathrm{C}|\right\}$ obtemos

$$
\left|c_{m}\right| \leqq \frac{M}{(1+|m|)^{k}} \text {, para todo } m e z^{n}
$$

Agora, se para cada $k \in \mathbb{Z}^{+}$existir $c>0$ tal que

$$
\left|c_{m}\right| \leqq \frac{c}{(1+|m|)^{k}}, \quad m \in \mathbb{z}^{n}
$$


então, como para $m \neq 0$ vale

$$
\frac{1}{(1+|m|)^{k}} \leqq \frac{1}{|m|^{k}}
$$

chegamos a

$$
\begin{aligned}
& \left|c_{m}\right| \leqq \frac{c}{|m|^{k}}, \quad m \in \mathbb{z}^{n}, \quad m \neq 0 . \\
& \text { o espaço das seqüēncias de decrescimento rápido será deno }
\end{aligned}
$$
tado por $s\left(\mathbb{Z}^{\mathrm{n}} ; \mathbb{C}\right)$ ou simplesmente $\mathrm{s}$.

DEFINIÇÃO 3 - Uma seqüência numérica $\left(c_{m}\right)_{m \in \mathbb{Z}^{\mathrm{n}}}$ é dita de crescimento lento se existirem constantes $M>0$ e $k$ e $\mathbb{Z}^{+}$ tais que

$$
\left|c_{m}\right| \leqq M|m|^{k} \quad \text { para todo } m \in \mathbb{z}^{n}, m \neq 0
$$

A definição acima é equivalente a existirem constantes $M>0$ e $k$ e $\mathbb{Z}^{+}$tais que

$$
\begin{aligned}
& \left|c_{m}\right| \leqq M(1+|m|)^{k} \text { para todo } m \in \mathbb{Z}^{n} . \\
& \text { Pois, se } M \text { e } k \text { são como na definição então } \\
& \left|c_{m}\right| \leqq M|m|^{k} \leqq M(1+|m|)^{k} \leqq M^{\prime}(1+|m|)^{k}
\end{aligned}
$$

onde $M^{\prime}=\max \left\{M,\left|c_{0}\right|\right\}$.

$$
\begin{aligned}
& \text { Agora, se existem } M>0 \text { e } k \in \mathbb{Z}^{+} \text {tais que } \\
& \left|c_{m}\right| \leqq M(1+|m|)^{k} \text { para todo } m e \mathbb{Z}^{n},
\end{aligned}
$$

temos que, se $m \neq 0$ então $|m| \geqq 1$ e

$$
\left|\mathrm{C}_{\mathrm{m}}\right| \leqq M(1+|\mathrm{m}|)^{\mathrm{k}} \leqq M(|\mathrm{~m}|+|\mathrm{m}|)^{\mathrm{k}}=2^{\mathrm{k}} \mathrm{M}|\mathrm{m}|^{\mathrm{k}} .
$$


o espaço das seqüēncias de crescimento lento será denota do por $s^{\prime}\left(\mathbb{Z}^{n} ; \mathbb{C}\right)$ ou, simplesmente, $\mathbf{s}^{\prime}$.

TEOREMA 2 $\cdot$ Seja $\left(c_{m}\right)_{m} \in \mathbb{Z}^{n}$ uma seqüēnciā rapidamente decres cente.

Então $f=\sum_{m \in \mathbb{Z}^{n}} c_{m} e_{m}$ pertence a $P_{T}\left(\mathbb{R}^{n}\right)$

OBS. $e_{m}(x)=e^{i w m \cdot x}, x \in \mathbb{R}^{n}$.

\section{DEMONSTRAÇÃO:}

Considere $s_{p}(x)=\sum_{|m| \leqq p} c_{m} e_{m}(x)$.

Como $\left(c_{m}\right)$ é de decrescimento rápido existe $M_{1}>0$ tal que

$$
\left|c_{m} e_{m}(x)\right|=\left|c_{m}\right|<\frac{M_{1}}{(1+|m|)^{n+1}} \text { para todo } m \in \mathbb{z}^{n}
$$

Pelo critério de Weierstrass $s_{p}$ é uniformemente conver gente e $f(x)=\sum_{m} e_{z^{n}} c_{m} e_{m}(x)$ é contínua.

E claro que $f$ é periódica.

Agora,

$\frac{\partial}{\partial x_{j}} s_{p}(x)=\sum_{|m| \leqq p} i w m_{j} c_{m} e^{i w m x}$

Novamente, existe $M_{2}>0$ tal que

$\left|i w m_{j} c_{m} e_{m}(x)\right|=w\left|m_{j}\right|\left|c_{m}\right| \leqq w(1+|m|)\left|c_{m}\right| \leqq$

$\leqq(1+|m|) M_{2}(1+|m|)^{-n-2}=M_{2}(1+|m|)^{-n-1}$.

Assim, $g_{j}(x)=\lim \frac{\partial}{\partial x_{j}} s_{p}(x)$ é contínua. (a convergēn cia é uniformel. 
Sejam $x, h \in \mathbb{R}^{n}$ e $\gamma:[0,1] \rightarrow \mathbb{R}^{n}$ dada por $\gamma(t)=x+t h$. Então, $s_{p}(x+h)-s_{p}(x)=s_{p}(\gamma(1))-s_{p}(\gamma(0))=\int_{0}^{1}\left(s_{p} \circ \gamma\right)^{\prime}(t) d t=$
$\left.=\int_{0}^{1}<\operatorname{grad} s_{p}(\gamma(t)), \gamma^{\prime}(t)>d t=\int_{0}^{1}<\operatorname{grad} s_{p}(x+t h), h\right\rangle d t$. Defina $g=\left(g_{1}, \ldots, g_{n}\right)$.

Entāo, quando $p \rightarrow \infty$, vem:

$f(x+h)-f(x)=\int_{0}^{1}\langle g(x+t h), h\rangle d t=$

$=\sum_{j=1}^{n} h_{j} \int_{0}^{1} g_{j}(x+t h) d t=\sum_{j=1}^{n} h_{j} g_{j}(x)+$

$+\sum_{j=1}^{n} h_{j} \int_{0}^{1}\left(g_{j}(x+t h)-g_{j}(x)\right) d t$.

Agora, se $h \neq 0$, temos

$$
\begin{aligned}
& \left.\left|\sum_{j=1}^{n} h_{j} \int_{0}^{1}\right| g_{j}(x+t h)-g_{j}(x)\right) d t \mid \cdot\|h\|^{-1} \leqq \\
& \leqq \\
& \sum_{j=1}^{n}\left|h_{j}\right| \cdot\left|h \|^{-1} \max \right| g_{j}(x+t h)-g_{j}(x) \mid \leqq \\
& \leqq \\
& \sum_{j=1}^{n} \max \left|g_{j}(x+t h)-g_{j}(x)\right| \rightarrow 0
\end{aligned}
$$

quando $h \rightarrow 0$.

Logo, f é diferenciável e grad $f(x)=g(x)$. Daí, con cluímos que

$$
f \in C^{1} \quad \text { e } \quad \frac{\partial f}{\partial x_{j}}=g_{j} \text {. }
$$

Usando indução segue a tese. 
$\underline{\text { TEOREMA } 3} \cdot$ Seja $\phi \in \mathrm{P}_{\mathrm{T}}\left(\mathbb{R}^{\mathrm{n}}\right) \cdot$ Então

$$
c_{m}(\phi)=T^{-n} \int_{[0, T]^{n}} e_{-m}(x) \phi(x) d x
$$

forma uma seqüência rapidamente: decrescente e

$$
\phi(x)=\sum_{m \in \mathbb{Z}^{n}} c_{m}(\phi) e_{m}(x)
$$

DEMONSTRAÇÃO:

$$
\begin{aligned}
& T^{n} m_{j} c_{m}(\phi)=\int_{[0, T]^{n}} m_{j} e^{-i m w x} \phi(x) d x= \\
& \left.=-\frac{1}{w} \int_{[0, T]^{n}} D_{j} e^{-i w m x}\right) \phi(x) d x= \\
& =\frac{1}{w} \int_{[0, T]^{n}} e^{-i w m x} D_{j} \phi(x) d x=\frac{T^{n}}{w} c_{m}\left(D_{j} \phi\right) .
\end{aligned}
$$

Usando indução sobre $|\alpha|$ podemos obter

$$
m^{\alpha} c_{m}(\phi)=\left(\frac{1}{w}\right)|\alpha| c_{m}\left(D^{\alpha} \phi\right)
$$

Assim, para cada $\ell$ e $\mathbb{N}$

$$
\begin{aligned}
& |\mathrm{m}|^{\ell}\left|\mathrm{c}_{\mathrm{m}}(\phi)\right|=\sum_{|\alpha|=\ell} \frac{\ell !}{\alpha !}\left|\mathrm{m}^{\alpha} \mathrm{c}_{\mathrm{m}}(\phi)\right|= \\
& =\frac{1}{w^{\ell}}|\alpha|^{\sum}=\ell \frac{l !}{\alpha !}\left|c_{m}\left(D^{\alpha} \phi\right)\right|= \\
& =\frac{1}{w^{\ell}}|\alpha|^{\sum}=\ell \frac{\ell !}{\alpha !}\left|\int_{[0, T]^{n}} D^{\alpha} \phi(x) e^{-i w m x} d x\right| \leqq \\
& \leqq \frac{T^{n}}{w^{\ell}} \quad|\alpha|^{l}=\ell \frac{\ell !}{\alpha !} \sup \left|D^{\alpha} \phi\right|=M \text {. }
\end{aligned}
$$

Como $M$ não depende de $m,\left(c_{m}(\phi)\right) e s\left(\mathbb{Z}^{n} ; \mathbb{C}\right)$. 
Seja $\quad \psi=\sum_{m \in \mathbb{Z}^{n}} c_{m}(\phi) e_{m} \cdot$ Pelo teorema anterior $\psi \in \mathrm{P}_{\mathrm{T}}\left(\mathbb{R}^{\mathrm{n}}\right)$

$$
\begin{aligned}
& c_{m}(\psi)=\frac{1}{T^{n}} \int_{[0, T]^{n}} \psi(x) e^{-i w m x} d x= \\
& =\frac{1}{T^{n}} \int_{[0, T]^{n}} \ell e^{n} \mathbb{z}^{n} c_{\ell}(\phi) e^{i w(m-\ell) x} d x= \\
& =\frac{1}{T^{n}} \ell \sum_{\ell \mathbb{Z}^{n} c_{\ell}(\phi)} \int_{[0, T]^{n}} e^{i w(m-\ell) x} d x=\frac{c_{m}(\phi)}{T^{n}} \int_{[0, T]^{n}} d x= \\
& =c_{m}(\phi) .
\end{aligned}
$$

Deste modo, se provarmos que se $c_{m}(\phi)=c_{m}(\psi)$ implicar em $\phi=\psi$ o teorema estará demonstrado.

o próximo lema diz isto.

LEMA 1 (Unicidade). Seja $\phi \in \mathbb{P}_{\mathrm{T}}\left(\mathbb{R}^{\mathrm{n}}\right)$ tal que $c_{m}(\phi)=0$ para todo $m$ em $\mathbb{Z}^{\mathrm{n}}$. Então $\phi=0$.

\section{DEMONSTRAÇÃO:}

Suponha que tenhamos demonstrado o lema para toda

$\psi: \mathbb{R}^{n} \rightarrow \mathbb{R}, \quad \psi \in \mathrm{P}_{T}\left(\mathbb{R}^{n}\right)$

Se $\phi \in P_{T}\left(\mathbb{R}^{n}\right)$ então

$$
\psi=\phi+\bar{\phi}=2 \operatorname{Re}(\phi): \mathbb{R}^{\mathrm{n}} \rightarrow \mathbb{R}
$$

é tal que

$$
c_{m}(\psi)=c_{m}(\phi)+c_{m}(\bar{\phi})=c_{m}(\psi)+\overline{c_{-m}(\phi)}=0 \text {. }
$$


Assim, $\psi=0$ e, portanto, $\phi=i \xi$ onde $\xi: \mathbb{R}^{n}+\mathbb{R}$, $\xi \in P_{T}\left(\mathbb{R}^{n}\right)$. Mas

$$
\begin{aligned}
& c_{m}(\xi)=i c_{m}(\phi)=0 . \\
& \text { Logo, } \phi=i \xi=0 .
\end{aligned}
$$

Com isto podemos supor, sem perda de generalidade, que $\phi$ assume valores reais.

$$
\begin{aligned}
& \text { Se } p(x)=\sum_{j=1}^{k} a_{j} e^{i w m_{j} x}, \text { onde } a_{j} \in a \text {, então } \\
& \int_{B_{T}(a)} \phi p=\int_{B_{T}(a)} \phi \sum_{j=1}^{k} a_{j} e_{m_{j}}=\sum_{j=1}^{k} a_{j} \int_{B_{T}(a)} \phi e_{m_{j}}= \\
& =T^{n} \sum_{j=1}^{k} a_{j} c_{-m_{j}}(\phi)=0 .
\end{aligned}
$$

Observe que se mostrarmos que $\phi(0)=0$ então teremos mos trado que $\phi(x)=0, x \in \mathbb{R}^{n}$, pois,

$$
\begin{aligned}
& T^{n} c_{m}\left(T_{-x} \phi\right)=\int_{[0, T]^{n}}\left(T_{-x} \phi\right)(y) e^{-i w m y} d y= \\
& =\int_{[0, T]^{n}} \phi(x+y) e^{-i w m y} d y=\int_{B_{T}(x)} \phi(z) e^{-i w m(z-x)} d z= \\
& =T^{n} e^{i w m x} \cdot c_{m}(\phi)=0 .
\end{aligned}
$$$$
\text { Logo, } \quad\left(T_{-x} \phi\right)(0)=0, \text { i.é, }
$$$$
\phi(x)=0
$$

Mostremos, enfim, que $\phi(0)=0$.

Suponha que $\phi(0)=c \neq 0$.

Sem perda de generalidade, podemos supor $c>0$ pois, 


$$
c_{m}(-\phi)=-c_{m}(\phi)
$$

e

$$
(-\phi)(0)=0 \Leftrightarrow \phi(0)=0 .
$$

Como $\phi$ é contínua, existe $\delta>0$ tal que $0<\delta<\frac{T}{2}$ e $x \in[-\delta, \delta]^{n}$ implica em $\phi(x)>\frac{c}{2}>0$.

$$
\begin{aligned}
& \text { Defina } \quad p:\left[-\frac{T}{2}, \frac{T}{2}\right] \rightarrow \mathbb{R} \\
& t \longmapsto 1+\cos w t-\cos w \delta .
\end{aligned}
$$

Se $\delta \leqq|t| \leqq \frac{T}{2}$ então caso $\delta \leqq t \leqq \frac{T}{2}$ temos

$0<w \delta \leqq w t \leqq \pi$

$-1 \leqq \cos w t \leqq \cos w \delta<1$

$-1-\cos w \delta \leqq \cos w t-\cos w \delta \leqq 0<1-\cos w \delta$

$-1<-\cos w \delta \leqq p(t) \leqq 1<2-\cos w \delta$.

Como $p(t)=p(-t)$ segue-se que $|p(t)| \leqq 1, \delta \leqq|t| \leqq \frac{T}{2}$.

Se $0 \leqq|t| \leqq \delta \quad$ então

$0 \leqq|w t| \leqq w \delta<\pi$

e

$$
\begin{aligned}
& \text { cos wt }=\cos |w t| \geqq \cos w \delta . \\
& \text { Logo, } p(t)=1+\cos w t-\cos w \delta \geqq 1 . \\
& \text { Para cada } a>1 \text { defina } \\
& p_{a}(t)=p\left(\frac{t}{a}\right)=1+\cos \frac{w t}{a}-\cos w \delta .
\end{aligned}
$$


Sejam

$$
q_{a}=1+\cos \left(\frac{w \delta}{a}\right)-\cos w \delta=\min _{-\frac{\delta}{a} \leqq t \leqq \frac{\delta}{a}} p(t)
$$

e

$$
k=\max _{-\delta \leqq t \leqq \delta} p(t)=2-\cos w \delta>1
$$

Como

$$
\lim _{a \rightarrow+\infty} q_{a}=2-\cos w \delta=k>1
$$

então

$$
\lim _{a \rightarrow+\infty} \frac{k}{q_{a}}=1
$$

e, para $n \geq 1$

$$
\lim _{a \rightarrow+\infty}\left(\frac{k}{q_{a}}\right)^{2(n-1)}=1
$$

Por (1) existe $a_{1}$ tal que $a \geqq a_{1}$ implica em $q_{a}>k-\frac{k-1}{2}=\frac{k+1}{2}$ e por (2) existe $a_{2}$ tal que $a \geq a_{2}$ im plica em $\left(\frac{k}{q_{a}}\right)^{2(n-1)}<1+\frac{k-1}{2}=\frac{k+1}{2}$.

Seja $a_{0}=\max \left\{a_{1}, a_{2}\right\}$.

Então para $a \geqq a_{0}$ vale

$\left(\frac{k}{q_{a}}\right)^{2(n-1)}<\frac{k+1}{2}<q_{a}$.

Logo, $\frac{k}{q_{a}}<q_{a}^{\frac{1}{2(n-1)}}, \quad$ i.é. , $k<q_{a}^{1+\frac{1}{2(n-1)}}$

Deste modo,

$p(t) \leqq k \leqq q_{a}^{1+\frac{1}{2(n-1)}}$ para todo $t \in[-\delta, \delta]$ e se 
te $\left[-\frac{\delta}{a}, \frac{\delta}{a}\right]$ vale $q_{a} \leqq p(t) \leqq q_{a}{ }^{1+\frac{1}{2(n-1)}}$.

Fixemos $a, a \geqq a_{0}$.

Considere

$$
\begin{aligned}
& P(x)=p\left(x_{1}\right) \ldots p\left(x_{n}\right)= \\
& =\left(1+\cos w x_{1}-\cos w \delta\right) \ldots\left(1+\cos w x_{n}-\cos w \delta\right)= \\
& =\left(1+\frac{e^{i \omega e_{1} x}-e^{-i w e_{1} x}}{2}-\cos w \delta\right) \ldots \\
& \left.\ldots 11+\frac{e^{i w e_{n} x}-e^{-i w e_{n} x}}{2}-\cos w \delta\right) .
\end{aligned}
$$

Para cada $N$ e $\mathbb{N}$ defina

$$
\begin{aligned}
& P_{N}(x)=(P(x))^{N} \cdot \\
& P_{N} \text { é da forma } \sum_{j=1}^{k} a_{j} e^{i w m_{j} x} .
\end{aligned}
$$

Temos

$$
\int_{\left[-\frac{T}{2}, \frac{T}{2}\right]^{n}} \phi P_{N}=\int_{[-\delta, \delta]^{n}} \phi P_{N}+\int_{K} \phi P_{N}+\int_{Q} \phi P_{N}
$$

onde $k \subset\left[-\frac{T}{2}, \frac{T}{2}\right]^{n}$ è dado por $: \quad x \in k \Leftrightarrow\left|x_{j}\right| \geqq \delta$, $\forall j=1, \ldots, n$ e $Q \subset\left[-\frac{T}{2}, \frac{T}{2}\right]^{n}$ é dado por: $x \in Q \Leftrightarrow \exists i_{1}, i_{2}$ tais que $\left|x_{i_{1}}\right| \leqq \delta$ e $\left|x_{i_{2}}\right|>\delta$. (No caso $n=1, Q=\emptyset$ ) vale que $\left[-\frac{T}{2}, \frac{T}{2}\right]^{n}=[-\delta, \delta]^{n} \cup K \cup Q$, união disjunta. (a menos de conjuntos de medida nula). Se $x \in[-\delta, \delta]^{n}$ então $P_{N}(x)=p^{N}\left(x_{1}\right) \cdots p^{N}\left(x_{n}\right) \geqq 1^{n N}=1$ 
e $\phi(x) \geqq \frac{c}{2}$. Logo, $\phi P_{N}>0$ e

$$
\int_{[-\delta, \delta]^{n}} \phi P_{N} \geq \int_{\left[-\frac{\delta}{a}, \frac{\delta}{a}\right]^{n}} \phi P_{N} \geq \frac{c}{2} \cdot q_{a}^{n N} \cdot\left(\frac{2 \delta}{a}\right)^{n} .
$$

Se $x \in k$ então $\frac{T}{2} \geqq\left|x_{j}\right| \geqq \delta$ para todo $j=1, \ldots, n$. Assim $\left|p\left(x_{j}\right)\right| \leqq 1, j=1, \ldots, n$.

Logo, $\left|P_{N}(x)\right| \leqq 1 \quad e$

$\int_{K} \phi P_{N} \geqq-\int_{K}\left|\phi P_{N}\right|=-\int_{K}|\phi|\left|P_{N}\right| \geqq-\sup |\phi| \cdot T^{n}$.

Se $x \in Q$ existem $i_{1}$ e $i_{2}$ tais que $\left|x_{i_{1}}\right| \leq \delta_{1} \quad$ e $\quad\left|x_{i_{2}}\right|>\delta \quad$

Então, $\left|p\left(x_{i_{2}}\right)\right| \leqq 1 \quad e$

$\left|P_{N}(x)\right|=\left|p\left(x_{1}\right)\right|^{N} \ldots \mid p\left(x_{n}\right)^{N} \leqq$

$\leqq\left|p\left(x_{1}\right)\right|^{N} \ldots\left|p\left(x_{i_{2}-1}\right)\right| \cdot\left|p\left(x_{i_{2}+1}\right)\right|^{N} \ldots\left|p\left(x_{n}\right)\right|^{N} \leqq$

$\leqq q^{\left(1+\frac{1}{2(n-1)}\right)(n-1) N}=q_{a}^{\left(n-\frac{1}{2}\right) N}$.

pois, se $x_{j} \in[-\delta, \delta]$ então $p\left(x_{j}\right) \leqq q_{a}^{1+\frac{1}{2(n-1)}} \epsilon$ se $x_{j} \in\left[-\frac{T}{2},-\delta\right] \cup\left[\delta, \frac{T}{2}\right]$ então $\left|p\left(x_{j}\right)\right| \leqq 1 \leqq q_{a}^{1+\frac{1}{2(n-1)}}$.

Assim,

$\int_{Q} \phi P_{N} \geqq-\int_{Q}|\phi|\left|P_{N}\right| \geqq-\sup |\phi| \cdot q_{a}^{\left(n-\frac{1}{2}\right) N} T^{n}$.

Portanto, 


$$
\begin{aligned}
& \int_{\left[-\frac{T}{2}, \frac{T}{2}\right]^{n} \phi P_{N} \geqq \frac{c}{a}\left(\frac{2 \delta}{a}\right)^{n} q_{a}^{n N}-T^{n} \sup |\phi|-} \\
& -T^{n} \sup |\phi| q_{a}^{\left(n-\frac{1}{2}\right) N}= \\
& =q_{a}^{n N}\left(\frac{c}{2}\left(\frac{2 \delta}{a}\right)^{n}-\frac{\sup |\phi| \cdot T^{n}}{q_{a}^{n N}}-\frac{\sup |\phi| T^{n}}{q_{a}^{N / 2}}\right) .
\end{aligned}
$$

Como $q_{a}>1$ temos:

$$
\lim _{N \rightarrow \infty} q_{a}^{n N}\left(\frac{c}{2}\left(\frac{2 \delta}{a}\right)^{n}-\frac{\sup |\phi| \cdot T^{n}}{q_{a}^{n N}}-\frac{\sup |\phi| \cdot T^{n}}{q_{a}^{N / 2}}\right)=\infty \text {. }
$$

Logo, existe $N \in \mathbb{N}$ tal que $\int_{\left[-\frac{T}{2}, \frac{T}{2}\right]^{n}} \phi P_{N} \geqq 1$.

Absurdo.

Os Teoremas 2 e 3 permitem escrever um isomorfismo entre $s\left(\mathbb{Z}^{n} ; \mathbb{C}\right)$ e $P_{T}\left(\mathbb{R}^{n}\right):$

$$
\begin{aligned}
& A: s\left(\mathbb{Z}^{n} ; \mathbb{C}\right) \rightarrow P_{T}\left(\mathbb{R}^{n}\right) \\
& \left(c_{m}\right) \longmapsto \sum_{m} e_{z^{n}} c_{m} e_{m}
\end{aligned}
$$

A linearidade é imediata.

A sobrejetividade é dada pelo Teorema 3 , visto que, para cada $\phi \in \mathrm{P}_{\mathrm{T}}\left(\mathbb{R}^{\mathrm{n}}\right)$ a seqüência $\quad c=\left(c_{m}(\phi)\right)$ é tal que $c \in s\left(\mathbb{Z}^{n} ; \mathbb{C}\right)$ e $A(c)=\phi$.

Para se verificar a injetividade observe que se $A(c)=$ $=\sum_{m \in \mathbb{Z}^{n}} c_{m} e_{m}=0 \quad$ então 


$$
\begin{aligned}
& 0=\frac{1}{T^{n}} \int_{[0, T]^{n}}\left(\sum_{m \in \mathbb{Z}^{n}} c_{m} e_{m}\right) e_{-l}= \\
& =\frac{1}{T^{n}} \sum_{m \in \mathbb{Z}^{n}} c_{m} \int_{\left[0, T^{n}\right]} e_{m-\ell}=c_{\ell} .
\end{aligned}
$$

Antes de fazermos a caracterização das distribuicões perí ódicas com as seqüências de crescimento lento, mostremos que $\mathrm{P}_{\mathrm{T}}^{\prime}$ é completo no seguinte sentido:

TEOREMA 4 $\cdot$ Se $\left(f_{j}\right)_{j \in \mathbb{N}}$ é una seqüência em $P_{T}^{\prime}$ tal que dados $\theta \in \mathrm{P}_{\mathrm{T}}$ e $\varepsilon>0$ existe $j_{0}$ e $\mathbb{N}$ tal que $j, k \geqq j_{0}$ implica que $\left|\left(f_{j}-f_{k}, \theta\right)\right|<\varepsilon$ então $f_{j} \rightarrow f$ em $P_{T}^{\prime}, i . e ́ .$, existe f $E P_{T}^{\prime}$ tal que $\left(f_{j}, \theta\right) \rightarrow(f, \theta)$ para cada $\theta \in P_{T}$.

\section{DEMONSTRAÇÃO:}

Mostremos antes que se $\left(\theta_{j}\right), \theta_{j}$ e $\mathrm{P}_{\mathrm{T}}$ é de Cauchy então $\theta_{j} \rightarrow \theta$ em $P_{T}$ para alguma $\theta$.

Como para cada $\mathrm{p} \in \mathbb{I N}$

$$
p_{k}\left(\theta_{j+p}-\theta_{j}\right)=\sum_{|\alpha| \leqq k} \sup \left|D^{\alpha} \theta_{j+p}-D^{\alpha} \theta_{j}\right| \rightarrow 0,
$$

quando $j \rightarrow \infty$ para todo $k \in \mathbb{N}, D^{\alpha} \theta_{j}$ converge uniformemen te para uma função T-periódica contínua $\theta_{\alpha}$.

$$
\begin{aligned}
& \text { Para cada } \psi \in \mathrm{P}_{\mathrm{T}} \\
& \left(\mathrm{D}^{\alpha} \theta_{0}, \psi\right)=(-1)|\alpha|\left(\theta_{0}, \mathrm{D}^{\alpha} \psi\right)=(-1)|\alpha| \int_{[0, T]^{\mathrm{n}}} \theta_{0} D^{\alpha} \psi= \\
& =\lim _{j \rightarrow \infty}(-1)|\alpha| \int_{[0, T]^{n}} \theta_{j} D^{\alpha} \psi=\lim _{j \rightarrow \infty} \int_{[0, T]^{\mathrm{n}}} \mathrm{D}^{\alpha} \theta_{j} \psi= \\
& =\int_{[0, T]^{\mathrm{n}}} \theta_{\alpha} \psi=\left(\theta_{\alpha}, \psi\right) .
\end{aligned}
$$


Usando o Teorema 4 da seção 2 repetidas vezes concluímos que $\theta_{\alpha} \in \mathrm{P}_{\mathrm{T}}$ e $\quad \mathrm{D}^{\alpha} \theta_{0}=\theta_{\alpha}$.

Concluimos então que $D^{\alpha}{ }_{j}+D^{\alpha} \theta_{0}$ uniformemente para cada $\alpha$, isto é,

$$
\theta_{j} \rightarrow \theta \quad \text { em } \quad P_{T}
$$

Considere a seqüēncia $\left(\rho_{j}\right)$ de funçōes contínuas, defini das em $\mathrm{P}_{\mathrm{T}}$, dadas por

$$
\rho_{j}(\theta)=\left|\left(f_{j}, \theta\right)\right|, j=1,2, \ldots
$$

Por uma aplicação do teorema de Baire [ ver [1], [7] ] existe uma bola aberta em $P_{T}, B\left(\theta_{1}, r\right)=\left\{\theta e P_{T} ; d\left(\theta, \theta_{1}\right)<r\right\}$ onde $\rho_{j}$ é uniformemente limitada, i.é., existe $M>0$ tal que $\left|\rho_{j}(\theta)\right| \leqq M$ para toda $\theta$ e $B\left(\theta_{1}, r\right)$ e je $\mathbb{N}$. Se $-\theta \in B\left(\theta_{1}, r\right)$ então $\rho_{j}(\theta)=\rho_{j}(-\theta) \leqq M$. seja $\theta \in B(0, r)$.

Escreva

$\theta=\frac{1}{2}\left[\left(\theta+\theta_{1}\right)+\left(\theta-\theta_{1}\right)\right]$

Temos

$$
d\left(\theta+\theta_{1}, \theta_{1}\right)=\sum_{k=1}^{\infty} \frac{p_{k}(\theta)}{2^{k}\left(1+p_{k}(\theta)\right)}=d(\theta, 0)<r
$$

e

$$
d\left(-\left(\theta-\theta_{1}\right), \theta_{1}\right)=d\left(\theta_{1}-\theta, \theta_{1}\right)=\sum_{k=1}^{\infty} \frac{p_{k}(\theta)}{2^{k}\left(1+p_{k}(\theta)\right)}=d(\theta, 0)<r
$$


62

$$
\begin{aligned}
& \rho_{j}(\theta)=\left|\left(f_{j}, \frac{1}{2}\left[\left(\theta+\theta_{1}\right)+\left(\theta-\theta_{1}\right)\right]\right)\right| \leqq \\
& \leq \frac{1}{2}\left[\left|\left(f_{j}, \theta+\theta_{1}\right)\right|+\left|\left(f_{j}, \theta-\theta_{1}\right)\right|\right]= \\
& =\frac{1}{2}\left[\rho_{j}\left(\theta+\theta_{1}\right)+\rho_{j}\left(\theta-\theta_{1}\right)\right] \leqq M
\end{aligned}
$$

pois $\theta+\theta_{1}$ e $-\left(\theta-\theta_{1}\right)$ e $B\left(\theta_{1}, r\right)$.

Defina $f: P_{T} \rightarrow \mathbb{C}$ por $f(\theta)=\lim _{j \rightarrow \infty}\left(f_{j}, \theta\right)$. f está bem definida pois, para cada $\theta e \mathrm{P}_{\mathrm{T}}$, a seqüência $\left(f_{j}, \theta\right)$ é de Cauchy. Mostremos que $f \in \mathrm{P}_{\mathrm{T}}^{\prime}$.

Seja $\theta_{\ell} \rightarrow 0$ em $P_{\mathrm{T}}$

Para cada $\varepsilon>0$ tomemos $\ell_{0} \in \mathbb{N}$ tal que $\frac{2 M \theta_{\ell}}{\varepsilon} \in B(0, r)$ sempre que $\ell \geqq \ell_{0} \cdot$

Dessa forma

$$
\rho_{j}\left(\frac{2 M \theta_{\ell}}{\varepsilon}\right)=\frac{2 M}{\varepsilon}\left|\left(f_{j}, \theta_{\ell}\right)\right| \leqq M
$$

sempre que $\ell \geqq \ell_{0}, j=1,2, \ldots$, ou seja, para todo $j \in \mathbb{N}$ e $\quad l \geqq \ell_{0}$

$$
\left|\left(f_{j}, \theta_{\ell}\right)\right| \leqq \frac{\varepsilon}{2}
$$

Como $f\left(\theta_{\ell}\right)=\lim _{j \rightarrow \infty}\left(f_{j}, \theta_{\ell}\right)$, para cada $\ell \geqq \ell_{0}$ existe $j_{\ell}$ tal que

$$
\begin{aligned}
& \left|f\left(\theta_{\ell}\right)-\left(f_{j_{\ell}}, \theta_{\ell}\right)\right|<\frac{\varepsilon}{2} . \\
& \text { Assim, para } \ell \geqq \ell_{O} \\
& \left|f\left(\theta_{\ell}\right)\right| \leqq\left|f\left(\theta_{\ell}\right)-\left(f_{j_{\ell}}, \theta_{\ell}\right)\right|+\left|\left(f_{j_{\ell}}, \theta_{\ell}\right)\right|<\frac{\varepsilon}{2}+\frac{\varepsilon}{2}=\varepsilon . \\
& \text { Portanto, } f \in P_{T}^{\prime} .
\end{aligned}
$$


TEOREMA 5 - Seja $\quad\left(c_{m}\right)_{m \in \mathbb{Z}^{n}}$ uma seqüência de crescimento lento.

Então a sērie $\sum_{m \in \mathbb{Z}^{n}} c_{m} e^{i w m \cdot x}, w=\frac{2 \pi}{T} \quad \bar{e}$ convergente em $P_{T}^{\prime}\left(\mathbb{R}^{n}\right)$ e se

$$
\begin{aligned}
& f=\sum_{m e} \mathbb{z}^{n} c_{m} e_{m}, \text { onde } e_{m}(x)=e^{i w m \cdot x}, \text { temos que } \\
& c_{m}=\frac{1}{T^{n}}\left(f, e_{-m}\right)=c_{m}(f) .
\end{aligned}
$$

\section{DEMONSTRAÇÃO:}

$$
\begin{aligned}
& \text { Seja } \theta \in P_{T}\left(\mathbb{R}^{n}\right) . \\
& \text { Para cada } m=\left(m_{1}, \ldots, m_{n}\right) \text { e } \mathbb{Z}^{n} \text { temos } \\
& m_{j}\left(e_{m}, \theta\right)=\int_{[0, T]^{n}} m_{j} e^{i w m x} \theta(x) d x=\frac{1}{w} \int_{[0, T]^{n}} D_{j}\left(e^{i w m x}\right) \theta(x) d x= \\
& =-\frac{1}{w} \int_{[0, T]^{n}} e^{i w m x} D_{j} \theta(x) d x .
\end{aligned}
$$$$
\text { Obtemos } m_{j}\left(e_{m}, \theta\right)=\frac{1}{w}\left(D_{j} e_{m}, \theta\right)=-\frac{1}{w}\left(e_{m}, D_{j} \theta\right) .
$$$$
\text { Usando indução sobre }|\alpha| \text { podemos ver que }
$$$$
m^{\alpha}\left(e_{m}, \theta\right)=\frac{1}{w|\alpha|}\left(D^{\alpha} e_{m}, \theta\right)=\frac{(-1)|\alpha|}{w|\alpha|}\left(e_{m}, D^{\alpha} \theta\right) \text {. }
$$$$
\text { Como }\left(c_{m}\right)_{m \in \mathbb{Z}^{n}} \text { é de crescimento lento, existem } M>0
$$
e $k \in \mathbb{N}$ tais que

$$
\left|c_{m}\right| \leqq M|m|^{k}, m \in \mathbb{z}^{n}, m \neq 0 \text {. }
$$


64

$$
\begin{aligned}
& =\sum_{|\alpha|=k+n+1} \frac{k !}{\alpha !} \cdot \frac{1}{w|\alpha|}\left|\left(e_{m}, D^{\alpha} \theta\right)\right| \leqq \sum_{|\alpha|=k+n+1} \frac{k !}{\alpha !} \frac{1}{w|\alpha|} \\
& \cdot \int_{[0, T]^{n}}\left|e^{i w m x} D^{\alpha} \theta(x)\right| d x=\sum_{|\alpha|=k+n+1} \frac{k !}{\alpha !} \cdot \frac{1}{w|\alpha|} \cdot \\
& \int_{[0, T]^{n}}\left|D^{\alpha} \theta(x)\right| d x=c .
\end{aligned}
$$

Observe que $C$ não depende de $m$ e se $\theta_{j} \rightarrow 0$ em $P_{T}$ então para cada $k \in \mathbb{N}|m|^{k+n+1}\left|\left(e_{m}, \theta_{j}\right)\right| \rightarrow 0$, para todo m e $\mathbb{z}^{\mathrm{n}}$.

$$
\begin{aligned}
& \left|c_{m}\left(e_{m}, \theta\right)\right|=\left|c_{m}\right|\left|\left(e_{m}, \theta\right)\right| \leqq M|m|^{k} \cdot \frac{c}{|m|^{k+n+1}}= \\
& =\frac{M C}{|m|^{n+1}}=\frac{\tilde{c}}{|m|^{n+1}}, \quad m e \mathbb{Z}^{n}, m \neq 0 .
\end{aligned}
$$

Considere a reduzida

$$
s_{j}(x)=\sum_{|m| \leqq j} c_{m} e^{i w m x}
$$

Para cada $p \in \mathbb{N}$

$$
\begin{aligned}
& \left|\left(s_{j+p}-s_{j}, \theta\right)\right|=\left|\left(\sum_{j+1 \leqq|m| \leqq j+p} c_{m} e_{m}, \theta\right)\right| \leqq \\
& \leq \sum_{j+1 \leqq|m| \leqq j+p}\left|c_{m}\left(e_{m}, \theta\right)\right| \leqq \sum_{j+1 \leqq|m| \leqq j+p} \frac{1}{|m|^{n+1}} .
\end{aligned}
$$

Como a série $\sum_{\mathrm{e}} \mathbb{Z}^{\mathrm{n}} \frac{1}{|\mathrm{~m}|^{\mathrm{n}+1}}$ é convergente, é de cauchy. Logo, $s_{j}$ também é de Cauchy e como $\mathrm{P}_{\mathrm{T}}^{\prime}$ é completo, existe f e $\mathrm{P}_{\mathrm{T}}^{\prime}$ tal que 


$$
f=\lim _{j \rightarrow \infty} s_{j}=\sum_{m \in \mathbb{Z}^{n}} c_{m} e_{m}
$$

Ainda,

$$
\frac{1}{T^{n}}\left(f, e_{-m}\right)=\frac{1}{T^{n}} \sum_{\ell \in \mathbb{Z}^{n}} c_{\ell} \int_{[0, T]^{n}} e^{i w(\ell-m) x} d x
$$

Mas,

$$
\int_{[0, T]^{n}} e^{i \omega(\ell-m) x} d x=\left\{\begin{array}{lll}
0 & \text { se } & \ell \neq m \\
T^{n} & \text { se } & \ell=m
\end{array} .\right.
$$

Então

$$
c_{m}=\frac{1}{T^{n}}\left(f, e_{-m}\right)
$$

o que o teorema acima diz é que toda série trigonométrica tendo coeficientes de crescimento lento é convergente em $\mathrm{P}_{\mathrm{T}}^{\prime}$. Já o seguinte nos diz que toda distribuição periódica po de ser representada como uma série trigonométrica com coeficien te:s de crescimento lento.

TEOREMA 6 . Seja $f \in P_{T}^{\prime}\left(\mathbb{R}^{n}\right)$. Se $c_{m}(f)=\frac{1}{T^{n}}\left(f, e_{-m}\right)$ então $\left(c_{m}(f)\right)$ é de crescimento lento e

$$
f=\sum_{m \in \mathbb{Z}^{n}} c_{m}(f) e_{m} \quad \text { em } \quad P_{T}^{\prime}\left(\mathbb{R}^{n}\right)
$$

DEMONSTRAÇÃO:

Como $f \in \mathrm{P}_{\mathrm{T}}^{\prime}$ existem $c>0$ e $\mathrm{k}$ e IN tais que 


$$
\begin{aligned}
& \left|c_{m}(f)=\frac{1}{T^{n}}\right|\left(f, e_{-m}\right)\left|\leqq \frac{c}{T^{n}}\right| \alpha\left|\leqq k \sup _{x}\right| D^{\alpha} e_{m}(x) \mid= \\
& =\frac{c}{T^{n}}|\alpha| \leqq k \sup _{x}|w| \alpha\left|\cdot m^{\alpha} \cdot e_{m}(x)\right|=
\end{aligned}
$$

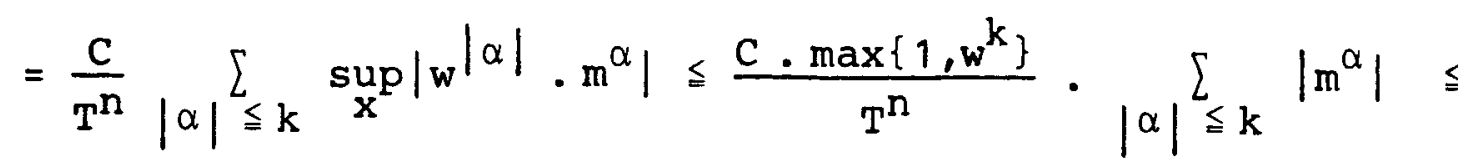

$$
\begin{aligned}
& \leqq C^{\prime} \quad \sum_{|\alpha| \leqq k}(1+|m|)|\alpha| \leqq c^{\prime \prime}(1+|m|)^{k} \text {, }
\end{aligned}
$$

para todo $m$ de $\mathbb{Z}^{\mathrm{n}}$.

Portanto $c_{m}(f)$ é de crescimento lento.

Pelo teorema anterior existe $g$ e $\mathrm{P}_{\mathrm{T}}^{\prime}$ tal que

$$
g=\sum_{m \in \mathbb{z}^{n}} c_{m}(f) e_{m}
$$

Resta-nos mostrar que $\mathrm{g}=\mathrm{f}$.

Para isto, mostremos primeiramente que $\delta=\frac{1}{\mathrm{~T}^{\mathrm{n}}} \sum_{\mathrm{m} \in \mathbb{Z}^{\mathrm{n}}} \mathrm{e}_{\mathrm{m}}$.

Para cada $\theta$ e $\mathrm{P}_{\mathrm{T}}$ temos

$$
\begin{aligned}
& \left(\frac{1}{T^{n}}|m| \leqq j\right. \\
& \left.-\theta(0)=\frac{1}{T^{n}} e_{m}-\delta, \theta\right)=\frac{1}{T^{n}} \sum_{|m| \leqq j} \int_{[0, T]^{n}} e_{m}(x) \theta(x) d x- \\
& -\frac{\theta(0)}{T^{n}}|m|_{[0, T]^{n}} e_{m}(x) \theta(x) d x- \\
& =\frac{1}{T^{n}} \mid m \int_{[0, T]^{n}} e_{m}(x) d x= \\
& =\frac{1}{T^{n}}|m|_{[0, T]^{n}} e_{m}(x)(\theta(x)-\theta(0) \mid d x= \\
& \int_{[0, T]^{n}}(\theta(x)-\theta(0)) e_{-m}(x) d x=
\end{aligned}
$$




$$
\begin{aligned}
& =\sum_{|m| \leqq j} c_{m}(\theta-\theta(0))=\sum_{|m| \leqq j} c_{m}(\theta-\theta(0)) e_{m}(0) \rightarrow \\
& \rightarrow(\theta-\theta(0))(0)=\theta(0)-\theta(0)=0 .
\end{aligned}
$$

Usando a continuidade seqüencial em cada variável da $\mathrm{T}-c o \underline{n}$ volução chegamos a

$$
\begin{aligned}
& (f, \theta)=(f \star \delta, \theta)=\left(\frac{1}{T^{n}} \sum_{m \in \mathbb{Z}^{n}} f \star e_{m}, \theta\right)= \\
& =\left(\frac{1}{T^{n}} \sum_{m} e_{\mathbb{Z}^{n}}\left(f,\left(e_{m}\right)_{a}^{v}\right), \theta(a)\right)= \\
& =\left(\frac{1}{T^{n}} \sum_{m} \mathbb{Z}^{n}\left(f, e_{-m}\right) \cdot e_{m}(a), \theta(a)\right)= \\
& =\left(\sum_{m} \mathbb{Z}^{n} c_{m}(f) e_{m}(a), \theta(a)\right)=(g, \theta) .
\end{aligned}
$$

Portanto, $f=g$.

Estes dois últimos teoremas mostram que é possivel estabe lecer um isomorfismo entre $s^{\prime}\left(\mathbb{Z}^{n} ; \mathbb{C}\right)$ e $\mathrm{P}_{T}^{\prime}\left(\mathbb{R}^{n}\right)$.

Defina

$$
\begin{aligned}
& B: s^{\prime}\left(\mathbb{Z}^{n} ; \mathbb{C}\right) \longrightarrow P_{T}^{\prime}\left(\mathbb{R}^{n}\right) \\
& c=\left(c_{m}\right) \longmapsto \\
& \sum_{m \in \mathbb{Z}^{n}} c_{m} e_{m}
\end{aligned}
$$

A linearidade de B é trivial.

A sobrejetividade decorre do último teorema, pois, para cada $f \in \mathrm{P}_{\mathrm{T}}^{\prime}\left(\mathbb{R}^{\mathrm{n}}\right)$ tome $\mathrm{c}=\left(\mathrm{c}_{\mathrm{m}}(\mathrm{f})\right) \mathrm{e}$, então, $\mathrm{B}(\mathrm{c})=f$.
A injetividade: 
68

Se $B(c)=\sum_{m=0} c_{m} e_{m}=0$ então 0 Teorema 5 diz que $c_{m}=\left(0, e_{-m}\right)=0, i . \bar{e}, \quad c=0$.

EXEMPLO 1 :

Já sabemos que $\delta=\frac{1}{\mathrm{~T}^{\mathrm{n}}} \sum_{\mathrm{m} \in \mathbb{Z}^{\mathrm{n}}} \mathrm{e}_{\mathrm{m}} ;$ calculemos a série de $D^{\alpha} \delta$

$$
\begin{aligned}
& c_{m}\left(D^{\alpha} \delta\right)=\frac{1}{T^{n}}\left(D^{\alpha} \delta, e_{-m}\right)=\frac{(-1)|\alpha|}{T^{n}}\left(\delta, D^{\alpha} e_{-m}\right)= \\
& =\frac{(-1)|\alpha|}{T^{n}}\left(\delta,(-1)|\alpha|_{w}|\alpha|_{m^{\alpha}} e_{-m}\right)=\frac{w|\alpha|}{T^{n}} m^{\alpha} .
\end{aligned}
$$

Logo,

$$
\mathrm{D}^{\alpha} \delta=\frac{1}{\mathrm{~T}^{\mathrm{n}}} \mathrm{m} \in \mathrm{z}^{\mathrm{n}}{ }^{\mathrm{w}|\alpha|} \mathrm{m}^{\alpha} e_{\mathrm{m}} .
$$

TEOREMA 7 $\cdot$ Se f,g e P

$$
f * g=T^{n} \sum_{m \in \mathbb{Z}^{n}} c_{m}(f) \cdot c_{m}(g) e_{m}
$$

DEMONSTRAÇÃO:

$$
\begin{aligned}
& c_{m}(f * g)=\frac{1}{T^{n}}\left(f * g, e_{-m}\right)=\frac{1}{T^{n}}\left(f * g * e_{m}\right)(0)= \\
& =\frac{1}{T^{n}}\left(f *\left(g * e_{m}\right)\right)(0)=\frac{1}{T^{n}}\left(f(x),\left(g * e_{m}\right)^{v}(x)\right)= \\
& =\frac{1}{T^{n}}\left(f(x),\left(g * e_{m}\right)(-x)\right)=\frac{1}{T^{n}}\left(f(x),\left(g(a), e_{m}(-x-a)\right)\right)= \\
& =\frac{1}{T^{n}}\left(f(x),\left(g(a), e_{-m}(a)\right) \cdot e_{-m}(x)\right)= \\
& =\frac{1}{T^{n}}\left(f(x), e_{-m}(x)\right)\left(g(a), e_{-m}(a)\right)=T^{n} c_{m}(f) \cdot c_{m}(g) .
\end{aligned}
$$


69

Logo,

$$
f * g=T^{n} \sum_{m \in z^{n}} c_{m}(f) \cdot c_{m}(g) e_{m}
$$

6. SERIE PARCIAL DE FOURIER

6.1. A SERIE PARCIAL DE FOURIER EM $\mathrm{P}_{\mathrm{T}}$ •

Sejam $p, q$ e $n$ em $\mathbb{I N}$ tais que

$$
\mathrm{n}=\mathrm{p}+\mathrm{q}
$$

Considere a soma direta $\mathbb{R}^{n}=\mathbb{R}^{p} \oplus \mathbb{R}^{q}$.

Escreveremos $(x, y) \in \mathbb{R}^{n}$ para indicar que $x$ e $\mathbb{R}^{p}$ e $y e \mathbb{R}^{q}$.

Dada $\phi \in \mathrm{P}_{\mathrm{T}}\left(\mathbb{R}^{\mathrm{n}}\right)$ considere para cada $\mathrm{x} \in \mathbb{R}^{\mathrm{P}}$ a função

$$
\psi_{\mathbf{x}}: \mathbb{R}^{\mathrm{q}} \rightarrow \mathbb{C}
$$

$$
y \longmapsto \phi(x, y)
$$

$$
\begin{aligned}
& \psi_{x} \in P_{T}\left(\mathbb{R}^{q}\right) \quad e \text {, portanto, } \\
& \qquad(x, y)=\psi_{x}(y)=\sum_{m} \sum_{\mathbb{Z}^{q}} \cdot c_{m}\left(\psi_{x}\right) e^{i w m y}
\end{aligned}
$$

onde

$$
\begin{aligned}
& c_{m}\left(\psi_{x}\right)=\frac{1}{T^{q}} \int_{[0, T]^{q}} \psi_{x}(y) e^{-i w m y} d y= \\
& =\frac{1}{T^{q}} \int_{[0, T]^{q}} \phi(x, y) e^{-i w m y} d y .
\end{aligned}
$$


Defina $\phi_{m}(x)=c_{m}\left(\psi_{x}\right)$.

Assim, $\phi(x, y)=\sum_{m \in \mathbb{Z}^{q}} \phi_{m}(x) e^{i w m y}$.

E claro que $\phi_{\mathrm{m}}$ é T-periódica.

$\phi_{\mathrm{m}} \quad$ é continua pois, se $\mathrm{x}_{j} \rightarrow \mathrm{x}_{\mathrm{o}}$ em $\mathbb{R}^{\mathrm{p}}$ então $\lim _{j \rightarrow \infty} \phi\left(x_{j}, y\right) e^{-i w m y}=\phi\left(x_{0}, y\right) e^{-i w m y}$ e como $\phi\left(x_{j}, y\right) e^{-i \omega m y}$ é mensurável e $\left|\phi\left(x_{j}, y\right) e^{-i w m y}\right|=\left|\phi\left(x_{j}, y\right)\right| \leqq c \quad$ ( onde $C$ não depende de $j$, pois $\phi$ é limitada), pelo Teorema da Con vergência Dominada temos que

$$
\begin{aligned}
& \lim _{j \rightarrow \infty} \phi_{m}\left(x_{j}\right)=T^{-q} \lim _{j \rightarrow \infty} \int_{[0, T]} \phi\left(x_{j}, y\right) e^{-i w m y} d y= \\
& =T^{-q} \int_{[0, T]} \phi\left(x_{0}, y\right) e^{-i w m y} d y=\phi_{m}\left(x_{0}\right) .
\end{aligned}
$$

Para verificarmos que $\phi_{\mathrm{m}}$ è $\mathrm{C}^{1}$ considere uma seqüência $\left(h_{j}\right), h_{j} \in \mathbb{R}^{*}, h_{j} \rightarrow 0$.

Temos que

$$
\begin{aligned}
& \lim _{j \rightarrow \infty}\left[\frac{\left.\phi\left(x+h_{j} e_{k}, y\right)-\phi(x, y)\right]}{h_{j}} \cdot e^{-i w m y}=\right. \\
& =\frac{\partial}{\partial x_{k}} \phi(x, y) e^{-i w m y}
\end{aligned}
$$

e

$$
\left|\frac{\partial \phi(x, y)}{\partial x_{k}} e^{-i v m y}\right|=\left|\frac{\partial \phi}{\partial x_{k}}(x, y)\right| \leqq c, \quad(x, y) e \mathbb{R}^{n} .
$$

Deste modo, a seqüência

$$
\frac{\phi\left(x+h_{j} e_{k}, y\right)-\phi(x, y)}{h_{j}} \cdot e^{-i w m y}
$$


também é limitada para todo $(x, y)$ e $\mathbb{R}^{n}$.

Pelo Teorema da Convergência Dominada segue-se que $\lim _{j \rightarrow \infty} \frac{\phi_{m}\left(x+h_{j} e_{k}\right)-\phi_{m}(x)}{h_{j}}=$

$=T^{-q} \lim _{j \rightarrow \infty} \int_{[0, T]} \frac{\phi\left(x+h_{j} e_{k}, y\right)-\phi(x, y)}{h_{j}} \cdot e^{-i w m y} d y=$

$=T^{-q} \int_{[0, T]^{q}} \frac{\partial}{\partial x_{k}} \phi(x, y) e^{-i w m y} d y$.

Isto é,

$\frac{\partial}{\partial x_{k}} \phi_{m}(x)=T^{-q} \int_{[0, T]^{q}} \frac{\partial}{\partial x_{k}} \phi(x, y) e^{-i w m y} d y$.

Como $\frac{\partial}{\partial x_{k}} \phi(x, y)$ é contínua, podemos usar o mesmo racio cínio feito na prova de que $\phi_{\mathrm{m}}$ é contínua para concluirmos que $\frac{\partial}{\partial x_{k}} \phi_{m}(x)$ também é.

Através de indução sobre a ordem da derivada concluímos que

$$
\phi_{m} \text { e } P_{T}\left(\mathbb{R}^{n}\right)
$$

$\underline{\text { TEOREMA } 1} \cdot$ Seja $\left(\phi_{m}\right)_{m \in \mathbb{Z}^{q}}$ uma seqüência de funções de $P_{T}\left(\mathbb{R}^{P}\right)$ tal que para cada $\alpha \in \mathbb{N}^{P}$ e $N \in \mathbb{N}$ existe $C>0$ tal que

$$
\left|D^{\alpha} \phi_{m}(x)\right| \leqq \frac{C}{(1+|m|)^{N}},
$$

para todo $m \in \mathbb{Z}^{q} \quad$ e $x \in \mathbb{R}^{\mathrm{p}}$.

$$
\text { Então, a função } \psi: \mathbb{R}^{p+q} \rightarrow \mathbb{C} \text { dada por }
$$


72

$$
\begin{aligned}
& \psi(x, y)=\sum_{m} e^{q} \phi_{m}(x) e^{i w m y} \text { está bem definida e pertence a } \\
& P_{T}\left(\mathbb{R}^{n}\right)
\end{aligned}
$$

DEMONSTRAÇÃO:

Como para cada $x \in \mathbb{R}^{\mathrm{p}}$.

$$
\begin{aligned}
& \sum_{m \in \mathbb{Z}^{q}} \phi_{m}(x) e^{i w m y} \quad \text { é convergente, } \psi \text { está bem definida. } \\
& \text { Seja } \quad \psi_{\mathrm{x}}: \mathbb{R}^{\mathrm{q}} \rightarrow \mathbb{C} \\
& y \longmapsto \psi(x, y) \\
& \psi_{\mathbf{x}} \quad \bar{e} \text { evidentemente periódica. } \\
& \psi_{\mathrm{X}} \text { pertence a } \mathrm{P}_{\mathrm{T}}\left(\mathbb{R}^{\mathrm{q}}\right) \text {, pois, dado } \mathrm{N} \in \mathbb{N} \text {, existe } \mathrm{C}>0
\end{aligned}
$$

tal que

$$
\begin{aligned}
& \left|c_{m}\left(\psi_{x}\right)\right|=\left|\phi_{m}(x)\right| \leqq \frac{c}{(1+|m|)^{N}} \\
& \text { Mostremos que } \psi \text { é contínua. } \\
& \text { Seja }\left(x_{0}, y_{0}\right) \text { e } \mathbb{R}^{n} \\
& \left|\psi(x, y)-\psi\left(x_{0}, y_{0}\right)\right|=\mid \sum_{m \in \mathbb{Z}^{q}}\left(\phi_{m}(x) e^{i w m y}-\right. \\
& \left.-\phi_{m}\left(x_{0}\right) e^{i w m y_{0}}\right)|=| \sum_{m \in \mathbb{Z}^{q}}\left[\left(\phi_{m}(x)-\phi_{m}\left(x_{0}\right)\right) .\right. \\
& \left.\cdot e^{i w m y}+\phi_{m}\left(x_{o}\right)\left(e^{i w m y}-e^{i w m y_{0}}\right)\right] \mid \leqq \\
& \leqq \sum_{m} \in \mathbb{Z}^{q}\left|\phi_{m}(x)-\phi_{m}\left(x_{o}\right)\right|+1 \sum_{m} \mathbb{Z}^{q} \phi_{m}\left(x_{o}\right) \cdot \\
& \cdot\left(e^{i w m y}-e^{i w m y_{O}}\right)\left|=\sum_{m \in \mathbb{Z}^{q}}\right| \phi_{m}(x)-\phi_{m}\left(x_{0}\right) \mid+ \\
& +\left|\psi_{x_{0}}(y)-\psi_{x_{0}}\left(y_{0}\right)\right|
\end{aligned}
$$


73

Como existe $C>0$ tal que

$$
\begin{aligned}
& \qquad\left|\phi_{m}(x)-\phi_{m}\left(x_{0}\right)\right| \leqq \frac{C}{(1+|m|)^{q+1}}, m \in \mathbb{Z}^{q}, \quad \text { a função } \\
& \qquad \sum^{\sum} \mathbb{Z}^{q}\left|\phi_{m}(x)-\phi_{m}\left(x_{o}\right)\right| \\
& \text { é continua. }
\end{aligned}
$$

Como $\psi_{\mathrm{x}_{0}}$ também é, dado $\varepsilon>0$ existe $\delta>0$ tal que $|(x, y)|<\delta$ implica que

$$
\begin{aligned}
& \left|\psi(x, y)-\psi\left(x_{0}, y_{0}\right)\right| \leqq \sum_{m \in \mathbb{Z}^{q}}\left|\phi_{m}(x)-\phi\left(x_{0}\right)\right|+ \\
& +\left|\psi_{x_{0}}(y)-\psi_{x_{0}}\left(y_{0}\right)\right|<\frac{\varepsilon}{2}+\frac{\varepsilon}{2}=\varepsilon . \\
& \text { Vejamos agora que } \psi \text { é } c^{1}
\end{aligned}
$$

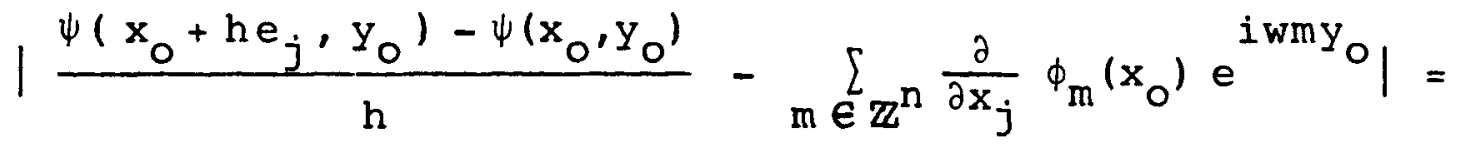

$$
\begin{aligned}
& =1 \frac{m \in \mathbb{Z}^{n} \phi_{m}\left(x_{0}+h e_{j}\right) e^{i w m y_{0}}-\sum_{m} e^{n} \phi_{m}\left(x_{o}\right) e^{i w m y_{0}}}{h}- \\
& -\sum_{m \in \mathbb{Z}^{n}} \frac{\partial}{\partial x_{j}} \phi_{m}\left(x_{o}\right) e^{i w m y_{0}}|=| \sum_{m \in \mathbb{Z}^{n}}\left[\frac{\phi_{m}\left(x_{0}+h e_{j}\right)-\phi_{m}\left(x_{0}\right)}{h}-\right. \\
& -\frac{\partial}{\partial x_{j}} \phi_{m}\left(x_{0}\right) j e^{i w m y_{0}}\left|\leqq \sum_{m \in \mathbb{Z}^{n}}\right| \frac{\phi_{m}\left(x_{0}+h e_{j}\right)-\phi_{m}\left(x_{0}\right)}{h}- \\
& -\frac{\partial}{\partial x_{j}} \phi_{m}\left(x_{0}\right)\left|=\sum_{m} \mathbb{z}^{n}\right| \frac{\partial \phi_{m}}{\partial x_{j}}\left(x_{o}+\xi_{m} e_{j}\right)-\frac{\partial \phi_{m}}{\partial x_{j}}\left(x_{0}\right) \mid=
\end{aligned}
$$


74

onde

$$
\left|\zeta_{m}\right|<\left|\xi_{m}\right|<|h|
$$

Existe $C>0$ tal que

$$
\left|\frac{\partial^{2}}{\partial x_{j}^{2}} \phi_{m}\left(x_{o}+\zeta e_{j}\right)\right| \leqq \frac{c}{(1+|m|)^{q+1}}, m \in \mathbb{z}^{q}
$$

Sendo assim, dado $\varepsilon>0$ tome

$$
\delta=\varepsilon \cdot\left(c \sum_{m} e_{\mathbb{Z}^{q}} \frac{1}{(1+|m|)^{q+1}}\right)^{-1}
$$

e se $|h|<\delta$ então $\left|\xi_{m}\right|<\delta$ e, portanto,

$$
\begin{aligned}
& \sum_{m \in \mathbb{Z}^{q}}\left|\frac{\partial^{2}}{\partial x_{j}^{2}} \phi_{m}\left(x_{o}+\zeta e_{j}\right)\right|\left|\xi_{m}\right|< \\
& <\sum_{m \in \mathbb{Z}^{n}\left|\frac{\partial^{2}}{\partial x_{j}^{2}} \phi_{m}\left(x_{o}+\zeta e_{j}\right)\right| \delta \leqq c \delta \sum_{m \in \mathbb{Z}^{q}} \frac{1}{(1+|m|)^{q+1}}=\varepsilon .}
\end{aligned}
$$

ou seja,

$$
\frac{\partial \psi}{\partial x_{j}}\left(x_{o}, y_{0}\right)=\sum_{m \in \mathbb{z}^{q}} \frac{\partial}{\partial x_{j}} \phi_{m}\left(x_{0}\right) e^{i w m y_{0}} .
$$

Como

$$
\left|\frac{\partial}{\partial x_{j}} \phi_{m}(x) e^{i w m y}\right|=\left|\frac{\partial}{\partial x_{j}} \phi_{m}(x)\right| \leqq \frac{c t e}{(1+|m|)^{q+1}}, m \in \mathbb{z}^{q}
$$

segue-se que $\frac{\partial \psi}{\partial x_{j}}$ é contínua, já que

$$
\lim _{h \rightarrow 0} \frac{\psi\left(x_{0}, y_{0}+h e_{j}\right)-\psi\left(x_{0}, y_{0}\right)}{h}=\lim _{h \rightarrow 0} \frac{\psi_{0}\left(y_{0}+h e_{j}\right)-\psi_{x_{0}}\left(y_{0}\right)}{h}=
$$


75

$$
\begin{aligned}
& =\frac{\partial}{\partial y_{j}} \psi_{x_{0}}\left(y_{0}\right)=\left.\frac{\partial}{\partial y_{j}}\left[\sum_{m \in \mathbb{Z}^{q}} \phi_{m}\left(x_{0}\right) e^{i w m y}\right]\right|_{y=y_{0}}= \\
& =i w \sum_{m \in \mathbb{Z}^{q}} m_{j} \phi_{m}\left(x_{0}\right) e^{i w m y_{0}}
\end{aligned}
$$

e

$$
\left|m_{j} \phi_{m}\left(x_{o}\right) e^{i w m y_{0}}\right|=\left|m_{j} \phi_{m}\left(x_{o}\right)\right| \leqq \frac{c t e}{(1+|m|)^{q+1}}, m \in \mathbb{z}^{q} .
$$

Mostremos por indução sobre a ordem das derivadas que $\psi$ e C .

Suponha que $\partial^{(\alpha, \beta)}$ é contínua e que

$$
\begin{aligned}
& \partial^{(\alpha, \beta)} \psi(x, y)=\sum_{m \in \mathbb{Z}^{q}} \partial^{\alpha_{\phi}}(x)(i w m)^{\beta} e^{i w m y} . \\
& \text { Defina } \alpha_{j}=\alpha+e_{j} \quad \text { e } \quad \alpha_{j j}=\alpha_{j}+e_{j} \\
& \frac{\partial^{(\alpha, \beta)} \psi\left(x_{0}+h e_{j}, y_{0}\right)-\partial^{(\alpha, \beta)} \psi\left(x_{0}, y_{0}\right)}{h}- \\
& -\sum_{m} e^{q}{z^{\alpha} j}^{\phi_{m}\left(x_{0}\right)(i w m)^{\beta}} e^{i w m y_{0}} \mid= \\
& =\mid \sum_{m \in \mathbb{Z}^{q}}\left[\frac{1}{h}\left(\partial^{\alpha} \phi_{m}\left(x_{o}+h e_{j}\right)-\partial^{\alpha} \phi_{m}\left(x_{o}\right)\right)-\right. \\
& \left.-\partial^{\alpha_{j}} \phi_{m}\left(x_{o}\right)\right](i w m)^{\beta} e^{i w m y_{0}} \mid \leqq \\
& \leqq \sum_{m \in \mathbb{Z}^{q}} \mid \frac{1}{h}\left(\partial^{\alpha} \phi_{m}\left(x_{o}+h e_{j}\right)-\partial^{\alpha} \phi_{m}\left(x_{o}\right)\right)- \\
& -\partial^{\alpha} j \phi_{m}\left(x_{0}\right)||(i w m) \beta \mid=
\end{aligned}
$$


76

$$
\begin{aligned}
& =\sum_{m} e_{z^{q}}\left|\left(\partial^{\alpha} \phi_{m}\left(x_{0}+\xi_{m} e_{j}\right)-\partial^{\alpha} \phi_{m}\left(x_{0}\right)\right)\right|\left|(i w m)^{\beta}\right|= \\
& =\sum_{m \in \mathbb{Z}^{q}}\left|\partial^{\alpha j j} \phi_{m}\left(x_{0}+\zeta_{m} e_{j}\right)\right|\left|\xi_{m}\right|\left|(i w m)^{\beta}\right| \\
& \text { onde }\left|\zeta_{m}\right|<\left|\xi_{m}\right|<|h| \text {. }
\end{aligned}
$$

Como existe $C>0$ tal que

$$
\left|\partial^{\alpha} j j \phi_{m}\left(x_{0}+\xi_{m} e_{j}\right)\right|\left|(i w m)^{\beta}\right| \leqq \frac{c}{(1+|m|)^{q+1}} \text {, }
$$

para todo $m \in \mathbb{Z}^{q}$, tome, para cada $\varepsilon>0$,

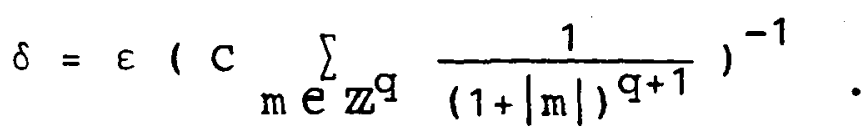

Então, se $h$ é tal que $|h|<\delta$ temos

$$
\sum_{m \in \mathbb{Z}^{q}}\left|\partial^{\alpha j j} \phi_{m}\left(x_{o}+\zeta_{m} e_{j}\right)\right|\left|\xi_{m}\right|\left|(i w m)^{\beta}\right|<\varepsilon .
$$

Dessa forma,

$$
\partial^{\left(\alpha_{j}, \beta\right)} \psi(x, y)=\sum_{m \in \mathbb{Z}^{q}} \partial^{\alpha_{j}} \phi_{m}(x) e^{i w m y} \cdot(i w m)^{\beta}
$$

e como

$$
\begin{aligned}
& \left|\partial^{\alpha_{j}} \phi_{m}(x) . e^{i w m y}(i w m)^{\beta}\right|= \\
& =\left|\partial^{\alpha_{j}} \phi_{m}(x)(i w m)^{\beta}\right| \leqq \frac{c t e}{(1+|m|)^{q+1}}, \quad m e \mathbb{Z}^{q}
\end{aligned}
$$

segue-se que 
77

$$
\begin{aligned}
& \partial^{\left(\alpha_{j}, \beta\right)} \psi \text { é continua. } \\
& \text { Defina } \beta_{j}=\beta+e_{j} \\
& \lim _{h \rightarrow 0}\left[\partial^{(\alpha, \beta)} \psi\left(x_{0}, y_{0}+h e_{j}\right)-\partial^{(\alpha, \beta)} \psi\left(x_{0}, y_{0}\right)\right] h^{-1}= \\
& =\lim _{h \rightarrow 0}\left[h^{-1} \sum_{m \in \mathbb{Z}^{q}} \partial^{\alpha} \phi_{m}\left(x_{0}\right)(i w m)^{\beta} e^{i w m y_{0}}\left(e^{i w h m e_{j}}-1\right)\right]= \\
& =\sum_{m} e^{q} \partial^{\alpha} \phi_{m}\left(x_{o}\right)(i w m)^{\beta}\left(i w m_{j}\right) e^{i w m y_{0}}= \\
& =\sum_{m \in \mathbb{Z}^{q}} \partial^{\alpha} \phi_{m}\left(x_{0}\right)(i w m)^{\beta} j e^{i w m y_{0}} .
\end{aligned}
$$

Deste modo, existe $\partial^{\left(\alpha, \beta_{j}\right)} \psi$ e é continua.

A periodicidade de $\psi$ é imediata.

No início desta seção vimos que se $\phi \in \mathrm{P}_{\mathrm{T}}\left(\mathbb{R}^{\mathrm{n}}\right)$ então

$$
\phi(x, y)=\sum_{m \in \mathbb{Z}^{q}} \phi_{m}(x) e^{i w m y}
$$

onde $\phi_{\mathrm{m}}$ e $\mathrm{P}_{\mathrm{T}}\left(\mathbb{R}^{\mathrm{p}}\right) \quad \mathrm{e}$

$$
\phi_{m}(x)=\frac{1}{T^{q}} \int_{[0, T]^{q}} \phi(x, y) e^{-i w m y} d y
$$

o teorema a seguir mostra que $\left(\phi_{m}\right)$ é uma sequiência de decrescimento rápido.

$\underline{\text { TEOREMA 2 }} \cdot$ Se $\phi \in \mathrm{P}_{\mathrm{T}}\left(\mathbb{R}^{\mathrm{n}}\right)$ então

$$
\phi(x, y)=\sum_{m \in \mathbb{Z}^{q}} \phi_{m}(x) e^{i w m y}
$$


78

onde

$$
\phi_{m} \text { e } P_{T}\left(\mathbb{R}^{p}\right), \quad \phi_{m}(x)=\frac{1}{T^{q}} \int_{[0, T]^{q}} \phi(x, y) e^{-i w m y} d y
$$

e dado $k \in \mathbb{N}$ e $\alpha \in \mathbb{N}^{P}$ existe $c>0$ tal que

$$
\begin{gathered}
\qquad\left|D^{\alpha} \phi_{m}(x)\right| \leq \frac{C}{(1+|m|)^{k}}, \\
\text { para todo } m \in \mathbb{Z}^{q} \text { e } x \in \mathbb{R}^{p} .
\end{gathered}
$$

DEMONSTRAÇAO:

Só resta demonstrar a última parte do teorema.

Sejam $\alpha \in \mathbb{N}^{p} \quad$ e $\quad \beta \in \mathbb{N}^{q}$

$$
\begin{aligned}
& T^{q}\left|m^{\beta} D^{\alpha} \phi_{m}(x)\right|=\left|\int_{[0, T]^{q}} D^{(\alpha, 0)} \phi(x, y) m^{\beta} e^{-i w m y} d y\right|= \\
& =\frac{1}{w|\beta|}\left|\int_{[0, T]^{q}} D^{(\alpha, 0)} \phi(x, y) \cdot D^{\beta} e^{-i w m y} d y\right|= \\
& =\frac{1}{w|\beta|}\left|\int_{[0, T]^{q}} D^{(\alpha, \beta)}(\phi(x, y)) e^{-i w m y} d y\right| .
\end{aligned}
$$

Dado $k \in \mathbb{N}$, temos

$$
\begin{aligned}
& \left.|m|^{k}\left|D^{\alpha} \phi_{m}(x)\right|=\left.\left.1\right|_{|B|}\right|_{=k} \frac{k !}{B !}\left|m^{\beta}\right|\right)\left|D^{\alpha} \phi_{m}(x)\right|= \\
& =|\beta|^{\sum}=k\left|\frac{k !}{\beta !} m^{\beta} \cdot D^{\alpha} \phi_{m}(x)\right|= \\
& =|\beta|^{\sum}=k \frac{k !}{\beta !} \cdot \frac{1}{w|\beta|}\left|\frac{1}{T^{q}} \int_{[0, T]^{q}} D^{(\alpha, \beta)}(\phi(x, y)) e^{-i w m y} d y\right| \\
& \leqq \frac{1}{w^{k}}|\beta|^{\sum}=k \frac{k !}{\beta !} \quad \sup _{(x, y) \in \mathbb{R}^{n}}\left|D^{(\alpha, \beta)} \phi(x, y)\right|=C(k, \phi) .
\end{aligned}
$$


Observe que $C(k, \phi)$ não depende de $m \in \mathbb{Z}^{q}$ e que se $\phi_{j} \rightarrow 0$ em $P_{T}$ então $C\left(k, \phi_{j}\right) \rightarrow 0$ para cada $k \in \mathbb{N}$.

Estes dois teoremas caracterizam também as funções de $\mathrm{P}_{\mathrm{T}}$ atravēs dos coeficientes (neste caso funções) de Fourier.

6.2. A SERIE PARCIAL DE FOURIER PARA DISTRIBUIÇÕES PERIODICAS.

Seja u $e P_{T}^{\prime}\left(\mathbb{R}^{p} \oplus \mathbb{R}^{q}\right), p+q=n$.

Para cada $\quad \gamma \in \mathrm{P}_{\mathrm{T}}\left(\mathrm{IR}^{\mathrm{P}}\right)$ defina

$v_{\gamma}: P_{T}\left(\mathbb{R}^{q}\right) \rightarrow \mathbb{C}$

$\theta \longmapsto v_{\gamma}(\theta)=(u, \gamma \otimes \theta)$

onde

$$
\begin{aligned}
\gamma \otimes \theta: & \mathbb{R}^{p} \oplus \mathbb{R}^{q} \rightarrow \mathbb{C} \\
(x, y) & \longmapsto \gamma(x) \cdot \theta(y) .
\end{aligned}
$$

Afirmação: $v_{\gamma}$ é linear, pois, para quaisquer $\theta_{1}, \theta_{2} \in P_{T}\left(\mathbb{R}^{q}\right)$ e $\quad a_{1}, a_{2}$ e $\mathbb{C}$ vale:

$$
\begin{aligned}
& v_{\gamma}\left(a_{1} \theta_{1}+a_{2} \theta_{2}\right)=\left(u, \gamma \otimes\left(a_{1} \theta_{1}+a_{2} \theta_{2}\right)\right)= \\
& =\left(u, a_{1} \gamma \otimes \theta_{1}+a_{2} \gamma \otimes \theta_{2}\right)=a_{1}\left(u, \gamma \otimes \theta_{1}\right)+a_{2}\left(u, \gamma \otimes \theta_{2}\right)= \\
& =a_{1} v_{\gamma}\left(\theta_{1}\right)+a_{2} v_{\gamma}\left(\theta_{2}\right) .
\end{aligned}
$$

$v_{\gamma}$ é contínuo. Seja $\theta_{j} \rightarrow 0$ em $P_{T}\left(\mathbb{R}^{p}\right)$. Então para todo $(\alpha, \beta) \in \mathbb{I N}^{p} \times \mathbb{N}^{q}$ 
80

$$
\begin{aligned}
& \left|D^{(\alpha, \beta)} \gamma \otimes \theta_{j}(x, y)\right|=\left|D^{(\alpha, \beta)} \gamma(x) \theta_{j}(y)\right|= \\
& =\left|D^{\alpha} \gamma(x) \cdot D^{\beta}{ }_{j}(y)\right| \leqq \sup \left|D^{\alpha} \gamma(x)\right| \cdot \sup \left|D^{\beta} \theta_{j}(y)\right| \rightarrow 0
\end{aligned}
$$

uniformemente.

Logo, $v_{\gamma}\left(\theta_{j}\right)=\left(u, \gamma \otimes \theta_{j}\right) \rightarrow 0$ em $\mathbb{C}$.

Assim, $v_{\gamma} \in P_{T}^{\prime}\left(\mathbb{R}^{q}\right) \quad e$, desta forma,

$$
v_{\gamma}=\sum_{m} \mathbb{Z}^{q} c_{m}\left(v_{\gamma}\right) e_{m}
$$

onde

$$
\begin{gathered}
c_{m}\left(v_{\gamma}\right)=\frac{1}{T^{q}}\left(v_{\gamma}, e_{-m}\right)=\frac{1}{T^{q}}\left(u, \gamma \otimes e_{-m}\right) \\
\text { Defina } u_{m}: P_{T}\left(\mathbb{R}^{p}\right) \rightarrow \mathbb{C} \\
\gamma \longmapsto c_{m}\left(v_{\gamma}\right)
\end{gathered}
$$

$$
\begin{aligned}
& u_{m} \text { é linear. } \\
& \qquad u_{m}\left(a_{1} \gamma_{1}+a_{2} \gamma_{2}\right)=\frac{1}{T^{q}}\left(u,\left(a_{1} \gamma_{1}+a_{2} \gamma_{2}\right) \otimes e_{-m}\right)= \\
& =\frac{a_{1}}{T^{q}}\left(u, \gamma_{1} \otimes e_{-m}\right)+\frac{a_{2}}{T^{q}}\left(u, \gamma_{2} \otimes e_{-m}\right)= \\
& =a_{1} u_{m}\left(\gamma_{1}\right)+a_{2} u_{m}\left(\gamma_{2}\right),
\end{aligned}
$$

para todos $\gamma_{1}, \gamma_{2} \in P_{T}\left(\mathbb{R}^{q}\right)$ e $a_{1}, a_{2} \in \mathbb{C}$.

Afirmação: $u_{m}$ é contínuo. Seja $\gamma_{j} \rightarrow 0$ em $P_{T}\left(\mathbb{R}^{P}\right) \cdot$ Então, para todo $(\alpha, \beta) \in \mathbb{N}^{\mathrm{P}} \times \mathbb{N}^{\mathrm{q}}$,

$$
\begin{aligned}
& \left|D^{(\alpha, \beta)} \gamma_{j} \otimes e_{-m}(x, y)\right|=\mid D^{\alpha} \gamma_{j}(x) D^{\beta} e_{-m}(y) \leqq \\
& \leqq \sup \left|D^{\beta} e_{-m}(y)\right| \cdot \sup \left|D^{\alpha} \gamma_{j}(x)\right|+0
\end{aligned}
$$


uniforme, quando $j \rightarrow \infty$, para cada $m \in \mathbb{Z}^{q}$.

Logo,

$$
u_{m}\left(\gamma_{j}\right)=\frac{1}{T^{q}}\left(u, \gamma_{j} \otimes e_{-m}\right)+0
$$

Assim, $u_{m} \in P_{T}^{\prime}\left(\mathbb{R}^{p}\right)$, para todo $m \in \mathbb{Z}^{q}$,

e

$$
\begin{aligned}
& v_{\gamma}=\sum_{m \in \mathbb{Z}^{q}}\left(u_{m}, \gamma\right) e_{m} \\
& (u, \gamma \otimes \theta)=\left(\sum_{m \in \mathbb{Z}^{q}}\left(u_{m}, \gamma\right) e_{m}, \theta\right) .
\end{aligned}
$$

Note que esta expressão já é suficiente para que u fi que bem definida, bastando tomar $\gamma(x)=e^{-i w m_{1} x} \quad e$ $\theta(y)=e^{-i w m_{2} y}$, onde $m=m_{1}+m_{2} \in \mathbb{z}^{n}, m_{1}$ e $\mathbb{z}^{p}$ e $m_{2}$ e $\mathbb{z}^{q}$. Dai,

$c_{m}(u)=(u, \gamma \otimes \theta)$

Vejamos agora como é o crescimento da seqüência $\left(u_{m}\right)$. Como $u \in P_{T}^{\prime}\left(\mathbb{R}^{p+q}\right)$ existem $C_{0}>0$ e $k_{0} \in \mathbb{N}$ tais que, para todo $\gamma \in \mathrm{P}_{\mathrm{T}}\left(\mathrm{IR}^{\mathrm{P}}\right)$

$$
\begin{aligned}
& \left|\left(u_{m}, \gamma\right)\right|=\frac{1}{T^{q}}\left|\left(u, \gamma \otimes e_{-m}\right)\right| \leqq \\
& \leqq c_{0}|(\alpha, \beta)| \leqq k_{0}\left|D^{(\alpha, \beta)}\left(\gamma(x) \cdot e_{-m}(y)\right)\right|= \\
& =c_{0}|(\alpha, \beta)| \leqq k_{0} \sup \left|D^{\alpha} \gamma(x)(-i w m)^{\beta} e^{-i w m y}\right|= \\
& =c_{0}|(\alpha, \beta)| \leqq k_{0} \sup \left|D^{\alpha} \gamma(x)\right| \cdot\left|(w m)^{\beta}\right| .
\end{aligned}
$$


Então, para algum $\mathrm{C}_{1}>0$

$$
\left|\left(u_{m}, \gamma\right)\right| \leqq c_{1} p_{k_{o}}(\gamma)(1+|m|)^{k_{o}}
$$

onde

$$
p_{k_{0}}(\gamma)=\sum_{|\alpha| \leqq k_{0}} \sup \left|D^{\alpha} \gamma\right| .
$$

Provamos o seguinte teorema

$\underline{\text { TEOREMA 3 }} \cdot$ Se $u \in P_{T}^{\prime}\left(\mathbb{R}^{P} \oplus \mathbb{R}^{q}\right)$ então

$$
u=\sum_{m \in \mathbb{Z}^{q}} u_{m} e_{m} \quad \text { onde } \quad u_{m} \in P_{T}^{\prime}\left(\mathbb{R}^{p}\right)
$$

é dada por $\left(u_{m}, \gamma\right)=c_{m}\left(v_{\gamma}\right)$ e

$$
c_{m}\left(v_{\gamma}\right)=\frac{1}{T^{q}}\left(u, \gamma \otimes e_{-m}\right), \gamma \in P_{T}\left(\mathbb{R}^{p}\right)
$$

Além do mais, para cada $\gamma \in \mathbb{P}_{T}\left(\mathbb{R}^{n}\right),\left(u_{m}, \gamma\right)$ é uma se qüência de crescimento lento.

$\underline{\text { TEOREMA } 4} \cdot$ Seja $\left(u_{m}\right)_{m \in \mathbb{Z}^{q}}$ uma seqüência de $P_{T}^{\prime}\left(\mathbb{R}^{\mathrm{p}}\right)$ que sa tisfaz a seguinte condição:

$$
\begin{aligned}
& \text { Existe } C>0 \text { e } k_{o} \text { e IN tal que } \\
& \left|\left(u_{m}, \phi\right)\right| \leqq C p_{k_{0}}(\phi)(1+|m|)^{k_{0}},
\end{aligned}
$$

para toda $\phi$ de $P_{-T}\left(\mathbb{R}^{p}\right)$ e todo $m$ de $\mathbb{Z}^{q}$. Então $u=\sum_{m \in \mathbb{Z}^{q}} u_{m} e_{m}$ pertence a $P_{T}^{\prime}\left(\mathbb{R}^{p+q}\right)$. 
83

DEMONSTRAÇÃO:

Para cada $\theta \in P_{T}\left(\mathbb{R}^{p+q}\right)$ considere a seqüência

$$
\begin{aligned}
& s_{j}=\sum_{|m| \leqq j}\left(u_{m}(x) e_{m}(y), \theta(x, y)\right)= \\
& m \in \mathbb{Z}^{q} \\
& =\sum_{m \in \mathbb{Z}^{q}} \sum_{\leqq j}\left(u_{m}(x), \int_{[0, T]^{q}} e_{m}(y) \theta(x, y) d y\right)= \\
& =\sum_{|m| \leqq j}\left(u_{m}(x), \theta_{m}(x)\right) . \\
& m \in \mathbb{z}^{q}
\end{aligned}
$$

Para cada $p \in \mathbb{N}$

$$
\begin{aligned}
& \left|s_{j+p}-s_{j}\right| \leqq \sum_{\substack{j<|m| \leqq j+p} \in \mathbb{Z}^{q}}\left|\left(u_{m}, \theta_{m}\right)\right| \leqq \\
& \leqq c \sum_{j<|m| \leqq j+p} p_{k_{o}}\left(\theta_{m}\right)(1+|m|)^{k_{0}} .
\end{aligned}
$$

Porém, como visto, $\theta_{m}$ é rapidamente decrescente; sendo assim,

$$
\begin{aligned}
& \mathrm{p}_{\mathrm{k}_{0}}\left(\theta_{\mathrm{m}}\right)=\sum_{|\alpha| \leqq \mathrm{k}_{0}} \sup \left|D^{\alpha} \theta_{\mathrm{m}}(\mathrm{x})\right| \leqq \sum_{|\alpha| \leqq \mathrm{k}_{0}} \mathrm{c}_{\alpha}(1+|\mathrm{m}|)^{-\mathrm{k}_{\mathrm{o}}-\mathrm{q}-1} \leqq \\
& \leqq c^{\prime}(1+|m|)^{-k_{0}-q-1} \text {. } \\
& \text { Assim, } \\
& \left|s_{j+p}-s_{j}\right| \leqq c c^{\prime} \sum_{j<|m| \leqq j+p}(1+|m|)-q-1 \rightarrow 0 \\
& m \in \mathbb{Z}^{q}
\end{aligned}
$$

quando $j \rightarrow \infty$.

Deste modo, $s_{j}$ é convergente, e $\sum_{m \in \mathbb{Z}^{q}} u_{m} e_{m}$ pertence a $\quad P^{\prime}\left(\mathbb{R}^{\mathrm{P}+\mathrm{q}}\right)$. 


\section{A PITULO II}

Neste capitulo serāo utilizadas basicamente as caracteri zações feitas no capitulo anterior (dos elementos de $\mathrm{P}_{\mathrm{T}} \mathrm{e} \mathrm{P}_{\mathrm{T}}^{\prime}$ com seus coeficientes de Fourier) para se analisar a regularida de (hipoeliticidade global) de alguns operadores diferenciais parciais lineares.

Na primeira seção será apresentada uma condição necessá ria e suficiente para que uma equação de T-convolução tenha sem pre uma solução. Estaremos assim incluindo a resolubilidade dos operadores diferenciais parciais lineares com coeficientes cons tantes.

Na segunda seção será mostrada uma condição necessária e suficiente para que um operador diferencial parcial linear a coeficientes constantes seja globalmente hipoelitico.

Com base neste resultado, a terceira seção será dedicada a operadores diferenciais globalmente hipoeliticos. Nesta seção serão vistos outros exemplos de como fica afetada a regularida de de alguns operadores pela adição de outros de ordem mais baixa.

A quarta seção diz respeito a um exemplo de operador glo balmente hipoelítico com coeficientes variáveis.

Por fim, na quinta seção será demonstrada hipoeliticidade global de certos operadores utilizando-se uma técnica diferente das usadas nas seções anteriores. 


\section{RESOLUBILIDADE DE EQUAÇÕES LINEARES DE T-CONVOLUÇÃO}

Na seção 4 do capitulo anterjor vimos que dada $f$ e $\mathrm{P}_{\mathrm{T}}^{\prime}$, a equação $f * u=g$ tem, para cada $g \in P_{T}^{\prime}$, uma solução em $\mathrm{P}_{\mathrm{T}}^{\prime}$ se, e somente se, existe a inversa de $f$ na $T$-convolução, isto é, $f * u=\delta$ tem solução em $P_{T}^{\prime}$.

o próximo teorema dá condições necessárias e suficientes sobre os coeficientes de Fourier de uma distribuição periódica para que ela possua uma inversa.

TEOREMA 1. Seja $f \in P_{T}^{\prime}\left(\mathbb{R}^{n}\right)$. Então, $f$ é inversível se, e somente se, $c_{m}(f) \neq 0$ para todo $m$ de $\mathbb{z}^{n}$ e $\left(\frac{1}{c_{m}(f)}\right)$ for mar uma seqüência de crescimento lento, i.é., existem $k \in \mathbb{N}$ e $c>0$ tais que

$$
\left|c_{m}(f)\right| \geq c(1+|m|)^{-k}, \quad m \in \mathbb{z}^{n}
$$

Antes da demonstração observemos que esta última condição sobre $c_{m}(f)$ diz que estes não podem decrescer rapidamente, i.é, $c_{m}(f) \notin s\left(\mathbb{Z}^{n} ; \mathbb{C}\right)$.

\section{DEMONSTRAÇÃO:}

Suponha que exista $f^{-1}$. Assim,

$$
c_{m}(\delta)=\frac{1}{T^{n}}=T^{n} \cdot c_{m}(f) \cdot c_{m}\left(f^{-1}\right)
$$

Desta forma, $c_{m}(f) \neq 0$, para todo $m \in \mathbb{Z}^{\mathrm{n}}$.

Como $\left(c_{m}\left(f^{-1}\right)\right)$ é de crescimento lento, pois $f^{-1} \in P_{T}^{\prime}$, 
vemos que $\frac{1}{c_{m}(f)}=\frac{c_{m}\left(f^{-1}\right)}{T^{2 n}}$ também o é.

Suponha agora que $c_{m}(f) \neq 0$ e que $\left(\frac{1}{c_{m}(f)}\right)$ seja de crescimento lento.

$$
\begin{aligned}
& \text { Defina } g=\frac{1}{T^{2 n}} m \in \mathbb{Z}^{n} \frac{1}{c_{m}(f)} \cdot e_{m}, g \in P_{T}^{\prime}\left(\mathbb{R}^{n}\right) \text { pois, } \\
& \left(c_{m}(g)\right)=\left(\frac{1}{T^{2 n}} \cdot \frac{1}{c_{m}(f)}\right) \in s^{\prime}\left(\mathbb{Z}^{n} ; \mathbb{C}\right) .
\end{aligned}
$$

Então,

$$
c_{m}(f * g)=T^{n} c_{m}(f) \cdot c_{m}(g)=T^{n} c_{m}(f) \cdot \frac{1}{T^{2 n} c_{m}(f)}=\frac{1}{T^{n}}=c_{m}(\delta)
$$

Logo, $f * g=\delta$

Por este teorema e o comentário que lhe precede, a equação $\mathrm{f} * \mathrm{u}=\mathrm{g}$ tem uma solução para cada $g$ e $\mathrm{P}_{\mathrm{T}}^{\prime}$ se, e somente se, $c_{m}(f) \neq 0$ e $\left(\frac{1}{c_{m}(f)}\right)$ é de crescimento lento.

Alēm do mais, já sabemos que caso exista a solução, ela é dada por $\mathrm{u}=\mathrm{f}^{-1} * \mathrm{~g}$.

Porém,

$$
c_{m}(u)=T^{n} c_{m}\left(f^{-1}\right) \cdot c_{m}(g)=\frac{1}{T^{n}} \frac{c_{m}(g)}{c_{m}(f)}
$$

e daí,

$$
u=\frac{1}{T^{n}} \sum_{m \in \mathbb{z}^{n}} \frac{c_{m}(g)}{c_{m}(f)} e_{m}
$$

Note que se $A=\left\{m \in \mathbb{Z}^{n} ; c_{m}(f)=0\right\}$ então a equação $f * u=g$ pode ter solução, dependendo da $g$.

Por exemplo, se $c_{m}(g)=0$ para todo $m \in A$ defina 
$u=\frac{1}{T^{n}} \sum_{m \in \mathbb{Z}^{n} \backslash A} \frac{c_{m}(g)}{c_{m}(f)} e_{m}$

Então, $\quad c_{m}(u)=\left\langle\begin{array}{ccc}\frac{c_{m}(g)}{T^{n} \cdot c_{m}(f)} & \text { se } \notin A \\ 0 & \text { se } & m \in A\end{array}\right.$.

Logo, $f * u=g$.

Neste caso não há unicidade de solução pois, basta acres centar à $u$ qualquer $\tilde{u} \in P_{T}^{\prime}\left(\mathbb{R}^{n}\right)$ da forma

$$
\tilde{u}=\sum_{j=1}^{k} e_{m_{j}}, \text { onde } m_{j} \in A, j=1, \ldots, k
$$

já que

$$
c_{m}(f \star \tilde{u})=T^{n} c_{m}(f) \cdot c_{m}(\tilde{u})=0
$$

pois, $c_{m}(f)=0$ se $m=m_{j}, j=1, \ldots, k$ e $c_{m}(\tilde{u})=0$ se $m \neq m_{j}, j=1, \ldots, k$.

Assim,

$f * \bar{u}=0$ e $f *(u+\tilde{u})=f * u+f * \tilde{u}=g$.

No caso em que $c_{m}(f) \neq 0$ para todo $m e \mathbb{Z}^{n}$ e $\left(\frac{1}{c_{m}(f)}\right)$ não é de crescimento lento, a equação $f \star u=g$ pode ter so lução desde que $\left(\frac{c_{m}(g)}{c_{m}(f)}\right)$ seja uma seqüência de crescimento lento. Neste caso, a solução será dada por:

$u=\frac{1}{T^{n}} \sum_{m \in \mathbb{Z}^{n}} \frac{c_{m}(g)}{c_{m}(f)} e_{m}$.

Por exemplo, se $f e P_{T}\left(\mathbb{R}^{n}\right)$ e $c_{m}(f) \neq 0$ então $\left(\frac{1}{c_{m}(f)}\right)$ 
não é de crescimento lento, porém, $f * \delta=f$.

Já vimos que, se $P(D)=\sum_{|\alpha| \leqq k} a_{\alpha} D^{\alpha}, a_{\alpha} e \mathbb{C}$ for um operador diferencial parcial linear a coeficientes constantes, a equação $P(D) u=g$, onde $g \in P_{T}^{\prime}\left(\mathbb{R}^{n}\right)$ pode ser transformada em

$$
u * P(D) \delta=g \text {. }
$$

Usando o Teorema 1 desta seção vemos que a equação admite uma única solução para cada $g$ se, e somente se, $g_{m}(P(D) \delta) \neq 0$, para todo $m$ de $\mathbb{z}^{\mathrm{n}}$ e $\left(\frac{1}{\mathrm{c}_{\mathrm{m}}(\mathrm{f})}\right)$ for uma seqüencia de cres cimento lento.

Note que, definindo $p(\xi)=\sum_{|\alpha| \leqq k} a_{\alpha} \xi^{\alpha}, \xi e \mathbb{R}^{n}, \circ$ simbolo de $P(D)$, temos:

$$
\begin{aligned}
& T^{n} c_{m}(P(D) \delta)=T^{n} c_{m}\left(\sum_{|\alpha| \leqq k} a_{\alpha} D^{\alpha} \delta\right)= \\
& =c_{m}\left(\sum_{|\alpha| \leqq k} \sum_{\ell \in z^{n}} a_{\alpha} w|\alpha| \ell^{\alpha} e_{\ell}\right)= \\
& =c_{m}\left(\sum_{\ell \in \mathbb{Z}^{n}}\left(\sum_{|\alpha| \leqq k} a_{\alpha} w^{|\alpha|} \ell^{\alpha}\right)_{e_{\ell}}\right)=|\alpha|_{\leqq k} a_{\alpha} \cdot w|\alpha| m^{\alpha}= \\
& =|\alpha|^{\sum} a_{\alpha} w|\alpha| m^{\alpha}=p(w m) .
\end{aligned}
$$

No caso de existência de solução ela será dada por

$$
u=\sum_{m \in \mathbb{Z}^{n}} \frac{c_{m}(q)}{p(w m)} e_{m} .
$$


EXEMPLO 2 :

Consj.dere

$P(D) u=u-\Delta u$,

onde

$\Delta=\left(\frac{\partial}{\partial x_{1}}\right)^{2}+\ldots+\left(\frac{\partial}{\partial x_{n}}\right)^{2}$

Temos

$p(\xi)=1+|\xi|^{2} \geqq 1$

e

$\frac{1}{p(w m)}=\frac{1}{w^{2}|m|^{2}+1} \leqq 1$

Então, para cada $g \in \mathrm{P}_{\mathrm{T}}^{\prime}$

$u=\frac{1}{T^{n}} \sum_{m \in \mathbb{Z}^{n}} \frac{c_{m}(g)}{w^{2}|m|^{2}+1} e_{m}$

é solução de $u-\Delta u=g$.

2. HIPOELITICIDADE GLOBAL

DEFINIÇÃO 1. Seja $P$ um operador diferencial parcial com coe ficientes em $P_{T}$. $P$ é dito globalmente hipoelítico se $u \in P_{T}^{\prime}$ e

$$
P u=f e P_{T} \text { então u } \in P_{T}
$$

TEOREMA 1 . No caso em que $P$ é um operador diferencial par 
cial linear a coeficientes constantes de ordem $k$ então a Defí nição 1 é equivalente a :

Se $u \in C_{T}^{k}$ e $P u=f \in P_{T}$ então $u \in P_{T}$.

DEMONSTRAÇÃO:

Suponha que $P$ satisfaz a Definição 1 .

Se $u \in C_{T}^{k}$ e $P u=f e P_{T}$ então $u \in P_{T}$ pois, $C_{T}^{k} \subset P_{T}$. Suponha que $P=\left.\right|_{|\alpha|} \sum_{\leqq k} a_{\alpha} D^{\alpha}$ näo satisfaz a Definição 1, isto é, existe

$$
u=\sum_{m \in \mathbb{Z}^{n}} c_{m} e_{m} e P_{T}^{\prime} \mid P_{T}
$$

tal que

$$
\mathrm{Pu}=\sum_{\mathrm{m} \in \mathbb{z}^{\mathrm{n}}}\left(\sum_{|\alpha| \leqq k} c_{m} \mathrm{~m}^{\alpha} \mathrm{a}_{\alpha}\right) \mathrm{e}_{\mathrm{m}}=f e \mathrm{P}_{\mathrm{T}}
$$

Como $u \in P_{T}^{\prime} \mid P_{T}$ existem $L, M_{1}, M_{2}>0$ e uma seqüência $\left(m_{k}\right)_{k}$ e IN tais que

$$
\frac{k}{\left(1+\left|m_{k}\right|^{2}\right)^{M_{1}}} \leqq\left|c_{m_{k}}\right|
$$

e

$$
\left|c_{m}\right| \leqq I\left(1+|m|^{2}\right)^{M_{2}}, \text { para todo } m \in \mathbb{Z}^{n}
$$

Como $f \in P_{T}$, para cada $M \in \mathbb{N}$ existe $K>0$ tal que

$$
\left|\sum_{|\alpha| \leqq k} c_{m} m^{\alpha} a_{\alpha}\right| \leqq \frac{K}{\left(1+|m|^{2}\right)^{M}} \text {. }
$$


Então, para todo $\mathrm{k}$ de $\mathbb{N}$

$$
\frac{k}{\left(1+\left|m_{k}\right|^{2}\right)^{M_{1}+M_{2}+p}} \leqq \frac{\left|c_{m_{k}}\right|}{\left(1+\left|m_{k}\right|^{2}\right)^{M_{2}+p}}
$$

e

$$
\frac{\left|c_{m}\right|}{\left(1+|m|^{2}\right)^{M_{2}+p}} \leqq \frac{L}{\left(1+|m|^{2}\right)^{p}} \quad \text { para todo } m \in \mathbb{Z}^{n}
$$

A primeira desigualdade garante que $v \notin \mathrm{P}_{\mathrm{T}}$ e a segunda, para $p$ suficientemente grande, que $v \in C_{T}^{k}$.

Assim,

$$
P(v)=\sum_{m \in \mathbb{Z}^{n}}\left(\sum_{|\alpha| \leqq k} \frac{c_{m} m^{\alpha} a_{\alpha}}{\left(1+|m|^{2}\right)^{M_{2}+p}}\right) e_{m}=g
$$

Afirmação: $\quad g$ e $\mathrm{P}_{\mathrm{T}}$.

De fato, se $M \in \mathbb{I}$ então

$$
\begin{aligned}
& \left|c_{m}(g)\right|=\left|\sum_{|\alpha|_{\leqq k}} \frac{c_{m} m^{\alpha} a_{\alpha}}{\left(1+|m|^{2}\right)^{M_{2}+p}}\right|= \\
& =\frac{1}{\left(1+|m|^{2}\right)^{M_{2}+p}}|| \sum_{\mid \alpha k} c_{m} m^{\alpha} a_{\alpha} \mid \leqq \\
& \left|\sum_{|\alpha| \leqq k} c_{m} m^{\alpha} a_{\alpha}\right| \leqq \frac{k}{\left(1+|m|^{2}\right)^{M}}
\end{aligned}
$$

TEOREMA 2 . Sejam $\mathrm{P}_{1}$ e $\mathrm{P}_{2}$ operadores globalmente hipoelítí cos. Então $\mathrm{P}_{1} \circ \mathrm{P}_{2}$ também $\circ$ é. 


\section{DEMONSTRAÇÃO:}

Se $u \in P_{T}^{\prime}$ e $\left(P_{1} \circ P_{2}\right)(u)=P_{1}\left(P_{2}(u)\right)=f$ e $P_{T}$ então, por $P_{1}$ ser globalmente hipoelitico, $P_{2} u$ e $P_{T} \cdot$ Por $P_{2}$ ser globalmente hipoelítico, $u \in \mathrm{P}_{\mathrm{T}}$.

TEOREMA 3 Sejam $\mathrm{P}_{1}$ e $\mathrm{P}_{2}$ operadores diferenciais parciais com coeficientes em $\mathrm{P}_{\mathrm{T}}$.

Então,

a) Se $P_{2}$ não é globalmente hipoelítico então $P_{1} \circ P_{2}$ tam bém não é.

b) Se $\mathrm{P}_{1}$ não é globalmente hipoelítico e $\mathrm{P}_{2}$ é resolúvel então $\mathrm{P}_{1} \circ \mathrm{P}_{2}$ não é globalmente hipoelítico.

\section{DEMONSTRAÇÃO:}

a) Existe $u \in P_{T}^{\prime} \backslash P_{T}$ tal que $P_{2} u=f e P_{T}$.

Como $\mathrm{P}_{1}$ tem coeficientes em $\mathrm{P}_{T}, \mathrm{P}_{1}\left(\mathrm{P}_{2}(\mathrm{u})\right)=\mathrm{P}_{1}$ f e $\mathrm{P}_{\mathrm{T}}$. Logo $\mathrm{P}_{1} \circ \mathrm{P}_{2}$ não é globalmente hipoelítico.

b) Como $P_{1}$ não é globalmente hipoelitico existe $u \in P_{T}^{\prime} \mid P_{T}$ tal que

$\mathrm{P}_{1} \mathrm{u}=\mathrm{f} \in \mathrm{P}_{\mathrm{T}}$

Como $\mathrm{P}_{2}$ é resolúvel, existe $\mathrm{v} \in \mathrm{P}_{\mathrm{T}}^{\prime}$ tal que $\mathrm{P}_{2} \mathrm{v}=\mathrm{u}$ : Note que $\mathrm{v} \notin \mathrm{P}_{\mathrm{T}}$ pois, caso contrário $\mathrm{u}=\mathrm{P}_{2} \mathrm{v} \in \mathrm{P}_{\mathrm{T}}$. Logo,

$v \in P_{T}^{\prime} \backslash P_{T} \quad$ e $\left(P_{1} \circ P_{2}\right) v=f e P_{T}$. 
$\underline{\text { TEOREMA } 4} \cdot$ Seja $P=\sum_{|\alpha| \leqq k} a_{\alpha} D^{\alpha}, a_{\alpha} e \mathbb{C}$

$P$ é globalmente hipoelitico (GH) se, e somente se, exis tem números reais positivos tais que

(LM)

$$
|P(m)| \geqq \frac{L}{\left(m_{1}^{2}+\ldots+m_{n}^{2}\right)^{M}}
$$

para $|m|$ suficientemente grande, onde $P(m)=|\alpha|_{\leqq k} a_{\alpha} m^{\alpha}$.

Antes da demonstração note que a condição (LM) é equiva lente a

$$
|P(m)| \geqq \frac{L}{|m|^{2 M}}, \text { para }|m|=\left|m_{1}\right|+\ldots+\left|m_{n}\right|
$$

suficientemente grande.

Pois,

$$
\begin{aligned}
& m_{1}^{2}+\ldots+m_{n}^{2} \leqq m_{1}^{2}+\ldots+m_{n}^{2}+2 \sum_{\substack{i=1 \\
i<j \leqq n}}^{n-1}\left|m_{i}\right|\left|m_{j}\right|= \\
& =|m|^{2} \leqq m_{1}^{2}+\ldots+m_{n}^{2}+\sum_{\substack{i=1 \\
i<j \leqq n}}^{n-1}\left(m_{i}^{2}+m_{j}^{2}\right)=n\left(m_{1}^{2}+\ldots+m_{n}^{2}\right) .
\end{aligned}
$$

DEMONSTRAÇÃ :

Suponha que existam $b, I$ e $M$ positivos tais que

$$
|P(m)| \geqq \frac{L}{|m|^{2 M}} \quad \text { se } \quad|m| \geqq b \text {. }
$$

Assim, se $P(m)=0$ implica que $m \in\left\{m \in \mathbb{Z}^{n} ;|m|<b\right\}=A$ que é finito. 
Seja $u=\sum_{m \in \mathbb{Z}^{n}} c_{m}(u) e_{m} e P_{T}^{\prime} \quad$ tal que $\quad P u=f \in P_{T}$. Assim,

$$
c_{m}(u) P(m)=c_{m}(f) \text {. }
$$

Dado $k \in \mathbb{N}$, para todo $m \in \mathbb{Z}^{n}$ tal que $|m| \geq b$, existe $C>0$ tal que

$$
\left|c_{m}(u)\right|=\left|\frac{c_{m}(f)}{P(m)}\right| \leqq\left|c_{m}(f)\right| \cdot \frac{|m|^{2 M}}{L} \leqq c \cdot|m|^{-k}
$$

pois, $c_{m}(f)$ é de decrescimento rápido.

Seja

$$
C^{\prime}=\max _{0<|m|<b}\left\{c,|m|^{k} \cdot\left|c_{m}(u)\right|\right\} .
$$

Então

$\left|c_{m}(u)\right| \leqq c^{\prime}|m|^{-k}, \quad$ para todo $m \in \mathbb{z}^{n}, m \neq 0$

Portanto, $\quad u \in P_{T}$.

Suponha que a condição (IM) seja falsa. Isto é, existe uma seqüência $\left(m_{j}\right)$ e $\mathbb{Z}^{n}$ tal que

$$
\left|P\left(m_{j}\right)\right| \leqq \frac{1}{\left|m_{j}\right|^{2 j}}
$$

e

$$
\begin{aligned}
& \left|m_{j}^{i}\right|+\infty \quad \text { para cada } i=1, \ldots, n \\
& \text { Seja } u=\sum_{j=1}^{\infty} e_{m_{j}} .
\end{aligned}
$$




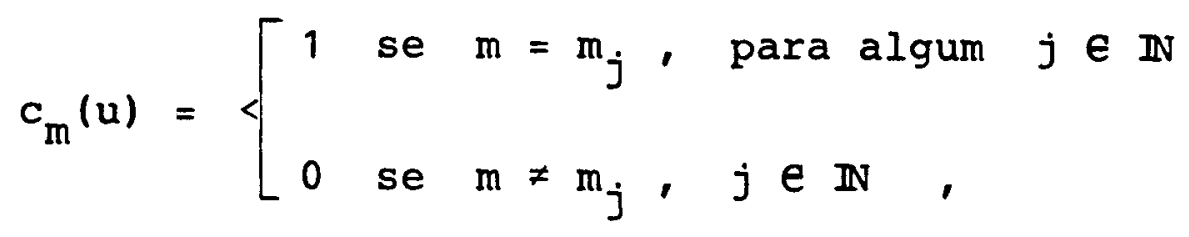

vemos que $\quad u \in P_{T}^{\prime}$.

Além do mais, $u \notin \mathrm{P}_{\mathrm{T}}$, jā que a subseqüência $\left(\mathrm{c}_{\mathrm{m}_{j}}(\mathrm{u})\right)$ não é de decrescimento rápido.

Porém,

$$
P(u)=\sum_{m \in \mathbb{Z}^{n}} c_{m}(u) P(m) e_{m}=\sum_{j=1}^{n} P\left(m_{j}\right) e_{m_{j}}=f
$$

e

$$
c_{m}(f)=<\left[\begin{array}{lll}
0 & \text { se } & m \neq m_{j}, j \in \mathbb{N} \\
P\left(m_{j}\right) & \text { se } m=m_{j}, \text { para algum } j \in \mathbb{N} .
\end{array}\right.
$$

Assim, dado $k \in \mathbb{N}$ temos, para todo $m \in \mathbb{Z}^{\mathrm{n}}, \mathrm{m} \neq 0$ que:

$$
\left|c_{m}(f)\right|=0 \quad \text { ou } \quad\left|c_{m}(f)\right|=|P(m)| \leqq \frac{1}{|m|^{2 k}}
$$

De qualquer forma

$$
\left|c_{m}(f)\right| \leqq \frac{1}{|m|^{2 k}}
$$

Portanto, f e $\mathrm{P}_{\mathrm{T}}$.

\section{EXEMPLOS DE OPERADORES GLOBALMENTE HIPOELITICOS}




$$
P=D_{1}-\alpha D_{2}, \alpha \in \mathbb{C}, D_{j}=\frac{1}{i} \frac{\partial}{\partial x_{j}} \quad j=1,2
$$

TEOREMA 1.

a) Se $a=a+i b, a, b \in \mathbb{R}, b \neq 0$ então $P$ é globalmen te hipoelitico.

b) Se $\alpha \in \mathbb{Q}$ então $P$ não é globalmente hipoelítico.

c) Se $\alpha \in \mathbb{R} \backslash \mathbb{D}$ então $\mathrm{P}$ é globalmente hipoelítico se, e somente se, $\alpha$ não é um número de Liouville.

OBSERVAÇÃO: Um número $\alpha \in \mathbb{R}$ é de Liouville se para todo $N \in \mathbb{N}$ existe $K>0$ e uma infinidade de pares $(n, m) \in \mathbb{Z}^{2}$ tais que $\left|\alpha-\frac{n}{m}\right|<\frac{K}{m^{N}}$.

DEMONSTRAÇÃO:

a) Para todo $(m, n) \in \mathbb{Z}^{2}$ tal que $|n| \geqq \frac{1}{|b|}$ temos $|P(m, n)|^{2}=|m-(a+i b) n|^{2}=|m-a n-i b n|^{2}=$ $=(m-a n)^{2}+b^{2} n^{2} \geqq b^{2} n^{2} \geqq 1$.

Pelo Teorema 4 da seção anterior segue-se que $P$ é global mente hipoelitico.

b) Seja $\alpha=\frac{m}{n}, m, n \in \mathbb{Z}, n \neq 0$.

Então, para qualquer inteiro $\ell$,

$P(\ell m, \ell n)=\ell m-\frac{m}{n} \cdot \ell n=0$.

Como $\mathrm{P}$ tem uma infinidade de zeros, pelo Teorema 4 se gue-se que $P$ não é globalmente hipoelitico. 
97

c) Se $\alpha \in \mathbb{R} \backslash \mathbb{Q}$ é de Liouville então para todo $N \in \mathbb{N}$ exí te $K>0$ e uma seqüência $\left(n_{j}, m_{j}\right)$ e $\mathbb{Z}^{2}$ onde podemos supor $m_{j}>0$ e $m_{j} \rightarrow+\infty$, tais que

$$
\left|\alpha-\frac{n_{j}}{m_{j}}\right|<\frac{K}{m_{j}^{N}} .
$$

Assim,

$$
\left|P\left(n_{j}, m_{j}\right)\right|=\left|\alpha m_{j}-n_{j}\right|<\frac{K}{m_{j}^{N-1}} . \quad(*)
$$

Como $m_{j}++\infty$ quando $j+\infty$, podemos supor que $\frac{k}{\mathrm{~m}_{j}^{N}}<1$. Desse modo,

$$
\left|n_{j}\right|-|\alpha| m_{j} \leqq\left|\alpha m_{j}-n_{j}\right|<\frac{k}{m_{j}^{N-1}}=m_{j} \frac{k}{m_{j}^{N}}<m_{j} \text {. }
$$

Logo,

$$
\left|n_{j}\right| \leqq(1+|\alpha|) m_{j}
$$

Daí,

$$
\begin{aligned}
& n_{j}^{2}+m_{j}^{2} \leqq\left[1+(1+|\alpha|)^{2}\right] m_{j}^{2}, \\
& \left(n_{j}^{2}+m_{j}^{2}\right)^{\frac{N-1}{2}} \leqq\left[1+(1+|\alpha|)^{2}\right]^{\frac{N-1}{2}} \cdot m_{j}^{N-1}
\end{aligned}
$$

e

$$
\frac{1}{m_{j}^{N-1}} \leqq \frac{\left[1+\left(1+|\alpha|^{2}\right]^{\frac{N-1}{2}}\right.}{\left(n_{j}^{2}+m_{j}^{2}\right)^{\frac{N-1}{2}}} \text {. }
$$

De $(*)$ segue que

$$
\left|P\left(n_{j}, m_{j}\right)\right|<\frac{K \cdot\left[1+(1+|\alpha|)^{2} j^{\frac{N-1}{2}}\right.}{\left(m_{j}^{2}+n_{j}^{2}\right)^{\frac{N-1}{2}}} \text {. }
$$$$
(* *)
$$ 
Assim, dados $L, M>0$ tome $N$ e IN tal que $\frac{N-3}{2}>M e$ e j suficier:temente grande para que ocorra

$$
\frac{K}{L} \cdot \frac{\left[1+(1+|\alpha|)^{2}\right]^{\frac{N-1}{2}}}{\left(m_{j}^{2}+n_{j}^{2}\right)^{\frac{N-1}{2}}}<1 .
$$

Deste modo, por $(* *)$

$$
\begin{aligned}
& \left|P\left(n_{j}, m_{j}\right)\right|<\frac{K}{L} \cdot \frac{\left[1+(1+|\alpha|)^{2}\right]^{\frac{N-1}{2}}}{\left(m_{j}^{2}+n_{j}^{2}\right)} \cdot \frac{L}{\left(m_{j}^{2}+n_{j}^{2}\right)^{\frac{N-3}{2}}}< \\
& <1 \cdot \frac{L}{\left(m_{j}^{2}+n_{j}^{2}\right)^{\frac{N-3}{2}}} \leqslant \frac{L}{\left(m_{j}^{2}+n_{j}^{2}\right)^{M}} \cdot
\end{aligned}
$$

Portanto, P não é globalmente hipoelítico.

Suponha agora que $\mathrm{P}$ não seja globalmente hipoelítico, i.é., P não satisfaz a condição (LM) do Teorema 4 da seção arterior.

Então, para todo $L>0$ e $M \in \mathbb{N}$ existe $\left(n_{M}, m_{M}\right) \in \mathbb{Z}^{2}$ tal que $\left|n_{M}\right| \rightarrow \infty, m_{M}>0$ e $m_{M} \rightarrow \infty$ quando $M \rightarrow \infty$ e

$$
\left|P\left(n_{M}, m_{M}\right)\right|<\frac{L}{\left(n_{M}^{2}+m_{M}^{2}\right)^{M}} \leqq \frac{L}{m_{M}^{2 M}}
$$

Logo,

$$
\left|\alpha m_{M}-n_{M}\right|<\frac{L}{m_{M}^{2 M}}
$$




$$
\left|\alpha-\frac{n_{M}}{m_{M}}\right|<\frac{L}{m_{M}^{2 M-1}}
$$

ou seja, a é de Liouville.

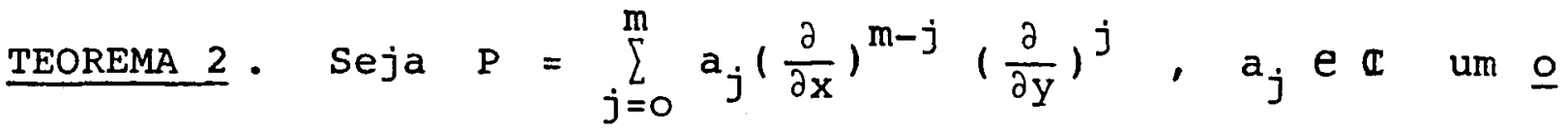
perador diferencial parcial a coeficientes constantes. Temos:

a) Se $a_{0}=a_{m}=0$ então $P$ não é globalmerte hipoelítico.

b) Se $a_{0} \neq 0$ (ou $a_{m} \neq 0$ ) então $P$ se escreve como $P=a_{0}\left(\frac{\partial}{\partial x}-\alpha_{1} \frac{\partial}{\partial y}\right) \ldots\left(\frac{\partial}{\partial x}-\alpha_{m} \frac{\partial}{\partial y}\right)$ $\left(\right.$ ou $\left.\quad p=a_{m}\left(\frac{\partial}{\partial y}-\beta_{1} \frac{\partial}{\partial x}\right) \ldots\left(\frac{\partial}{\partial y}-\beta_{m} \frac{\partial}{\partial x}\right)\right)$

e $P$ não é globalmente hipoelítico se, e somente se, exis te $\alpha_{i}$ (ou existe $\beta_{i}$ ) tal que $\alpha_{i}$ e (ou $\beta_{i} e$ ) ou $\alpha_{i}$ ( ou $\beta_{i}$ ) e IR $\backslash Q$ e é de Liouville.

\section{DEMONSTRAÇÃO:}

a) $P=\sum_{j=1}^{m-1} a_{j}\left(\frac{\partial}{\partial x}\right)^{m-j} \cdot\left(\frac{\partial}{\partial y}\right)^{j}$.

Seja $u=|\cos x| \in P_{2 \pi}^{\prime}\left(\mathbb{R}^{2}\right) \backslash P_{2 \pi}\left(\mathbb{R}^{2}\right) \quad$ como $\quad 1 \leqq j \leqq m-1$ temos

$\left(\frac{\partial}{\partial x}\right)^{m-j} \cdot\left(\frac{\partial}{\partial y}\right)^{j}|\cos x|=0$.

Logo

$$
\mathrm{Pu}=0 \in \mathrm{P}_{2 \pi}\left(\mathbb{R}^{2}\right)
$$


100

b) Se $a_{0} \neq 0$ então

$$
\begin{aligned}
& P(\xi, n)=\sum_{j=0}^{m} a_{j} \xi^{m-j} n^{j}=n^{m} \sum_{j=0}^{m} a_{j}\left(\frac{\xi}{n}\right)^{m-j}= \\
& =n^{m}\left[a_{0}\left(\frac{\xi}{n}\right)^{m}+a_{1}\left(\frac{\xi}{n}\right)^{m-1}+\ldots+a_{m}\right]= \\
& =n^{m} a_{0}\left(\frac{\xi}{n}-a_{1}\right) \ldots\left(\frac{\xi}{n}-\alpha_{m}\right)=a_{0}\left(\xi-\alpha_{1} n\right) \ldots\left(\xi-\alpha_{m} n\right) .
\end{aligned}
$$

e

$$
P=a_{0}\left(\frac{\partial}{\partial x}-\alpha_{1} \frac{\partial}{\partial y}\right) \ldots\left(\frac{\partial}{\partial x}-\alpha_{m} \frac{\partial}{\partial y}\right) .
$$

Seja $\alpha_{i} \in \mathbb{Q}$ ou $\alpha_{i} \in \mathbb{R} \backslash \mathbb{Q}$ de Liouville.

Como

$$
\left(\frac{\partial}{\partial x}-\alpha_{i} \frac{\partial}{\partial y}\right) \cdot\left(\frac{\partial}{\partial x}-\alpha_{j} \frac{\partial}{\partial y}\right)=\left(\frac{\partial}{\partial x}-\alpha_{j} \frac{\partial}{\partial y}\right)\left(\frac{\partial}{\partial x}-\alpha_{i} \frac{\partial}{\partial y}\right)
$$

temos:

$$
\begin{aligned}
& P=\left(\frac{\partial}{\partial x}-\alpha_{i} \frac{\partial}{\partial y}\right) \ldots\left(\frac{\partial}{\partial x}-\alpha_{i-1} \frac{\partial}{\partial y}\right) \cdot\left(\frac{\partial}{\partial x}-\alpha_{i+1} \frac{\partial}{\partial y}\right) \ldots \\
& \ldots\left(\frac{\partial}{\partial x}-\alpha_{m} \frac{\partial}{\partial y}\right)\left(\frac{\partial}{\partial x}-\alpha_{i} \frac{\partial}{\partial y}\right) .
\end{aligned}
$$

Existe $u \in P_{T}^{\prime} \backslash P_{T}$ tal que

$$
\left(\frac{\partial}{\partial x}-\alpha_{i} \frac{\partial}{\partial y}\right) u=g \in P_{T} .
$$

Desse modo,

$$
\begin{aligned}
& \mathrm{Pu}=\left(\frac{\partial}{\partial x}-\alpha_{1} \frac{\partial}{\partial y}\right) \ldots\left(\frac{\partial}{\partial x}-\alpha_{i-1} \frac{\partial}{\partial y}\right) \cdot\left(\frac{\partial}{\partial x}-\alpha_{i+1} \frac{\partial}{\partial y}\right) \ldots \\
& \ldots\left(\frac{\partial}{\partial x}-\alpha_{m} \frac{\partial}{\partial y}\right) g=f e P_{T} .
\end{aligned}
$$


Assim $P$ não é globalmente hipoelítico. Agora, se para todo $i=1, \ldots, n$ tivéssemos $\alpha_{i} \in \mathbb{C} \quad \mathbb{R}$ ou $\alpha_{i} \in \mathbb{R} \mathbb{D}$ não de Liouville então $P_{i}=\frac{\partial}{\partial x}-\alpha_{i} \frac{\partial}{\partial y}$ seria globalmente hipoeli tico. Como a composição de operadores globalmente hipoelíticos resulta num operador globalmente hipoelitico ( Teorema 3 da se ção anterior) teríamos

$$
\mathrm{P}=\mathrm{P}_{1} \circ \ldots \circ \mathrm{P}_{\mathrm{m}}
$$

globalmente hipoelitico.

Em alguns dos exemplos que seguem usaremos o fato de que se $p$ é um primo então $\alpha=\sqrt{p}$ é um irracional não de Liouville, pois, $\alpha$ é um número algēbrico [ver: [3] ].

\section{EXEMPLO 1 :}

Seja $\alpha=\sqrt{p}, p$ primo.

Como os operadores

$$
P_{1}=\alpha \frac{\partial}{\partial x}-\frac{\partial}{\partial y} \quad \text { e } \quad P_{2}=\alpha \frac{\partial}{\partial x}+\frac{\partial}{\partial y}
$$

são globalmente hipoelíticos, temos que

$$
\mathrm{P}=\mathrm{P}_{1} \circ \mathrm{P}_{2}=\alpha^{2} \frac{\partial^{2}}{\partial \mathrm{x}^{2}}-\frac{\partial^{2}}{\partial \mathrm{y}^{2}}
$$

tambēm o é.

EXEMPLO 2 :

$$
\text { Sejam } \quad \mathrm{P}_{2}=\alpha^{2} \frac{\partial^{2}}{\partial \mathrm{x}^{2}}-\frac{\partial^{2}}{\partial \mathrm{y}^{2}}, \alpha^{2}=\mathrm{p} \text { primo } \mathrm{e} \mathrm{P}_{\mathrm{o}}=-1 \text {. }
$$


Então $P=P_{2}+P_{0}=\alpha^{2} \frac{\partial^{2}}{\partial x^{2}}-\frac{\partial^{2}}{\partial y^{2}}-1$ não é (GH).

De fato, $P(m, n)=n^{2}-\alpha^{2} m^{2}-1=0$ (equação de Pell) tem infinitas soluçōes [ ver: [6] ].

$$
\begin{aligned}
& \text { Por exemplo, se } \alpha=\sqrt{2} \\
& n_{k}=\sum_{\substack{j=0 \\
j \text { par }}}^{k}\left(\begin{array}{l}
k \\
j
\end{array}\right) 3^{k-j} 2^{\frac{3}{2} j} \\
& m_{k}=\sum_{j=1}^{j=1}{ }_{j=1}^{k}\left(\begin{array}{l}
k \\
j
\end{array}\right) 3^{k-j} 2^{\frac{3 j-1}{2}} \\
& k=1,2,3, \ldots
\end{aligned}
$$

Este exemplo ilustra o fato de que se a um operador glo balmente hipoelitico de segunda ordem for adicionado um opera dor de ordem zero, o resultado pode ser um operador não regular.

\section{EXEMPLO 3 :}

Sejam $P_{2}=\alpha^{2} \frac{\partial^{2}}{\partial x^{2}}-\frac{\partial^{2}}{\partial y^{2}}, \alpha^{2}=p$, primo $P_{1}=2 i \frac{\partial}{\partial y}$.

Então $P=P_{2}+P_{1}$ não é globalmente hipoelítico. De fato,

$$
P(m, n)=-\alpha^{2} m^{2}+n^{2}-2 n=(n-1)^{2}-\alpha^{2} m^{2}-1=0
$$

tem infinitas soluções.

$$
\begin{aligned}
& \text { No caso } \alpha=\sqrt{2} \\
& n_{k}=1+\sum_{\substack{j=0 \\
j \text { par }}}^{k}\left(\begin{array}{l}
k \\
j
\end{array}\right) 3^{k-j} 2^{\frac{3 j}{2}}
\end{aligned}
$$




$$
\begin{aligned}
& m_{k}=\sum_{j=1}^{j=1} \sum_{j}^{k}\left(j_{j}^{k}\right) 3^{k-j} 2^{\frac{3 k-1}{2}} \\
& k=1,2, \ldots .
\end{aligned}
$$

Assim, se adicionarmos a um operador globalmente hipoeli tico de segunda ordem um operador de primeira ordem, o resultado poderá ser não globalmente hipoelítico.

\section{EXEMPLO 4 :}

Seja $\mathrm{P}_{\mathrm{N}}$ um operador diferencial parcial linear a coefi cientes constantes reais, homogêneo, de ordem par e GH.

Se $P_{1}=\alpha_{1} \frac{\partial}{\partial x_{1}}+\ldots+\alpha_{n} \frac{\partial}{\partial x_{n}}, \alpha_{j} \in \mathbb{R}, j=1,2, \ldots, n$ então $P=P_{N}+P_{1}$ é globalmente hipoelítico.

De fato, usando a condição (LM) do Teorema 4 da seção anterior, temos:

$$
\begin{aligned}
& P(m)=P_{N}(m)+i\left(\alpha_{1} m_{1}+\ldots+\alpha_{n} m_{n}\right) \\
& |P(m)|=\sqrt{\left(P_{N}(m)\right)^{2}+\left(\alpha_{1} m_{1}+\cdots+\alpha_{n} m_{n}\right)^{2}} \geqq \\
& \geqq\left|P_{N}(m)\right| \geqq \frac{L}{|m|^{2} M}
\end{aligned}
$$

para $|\mathrm{m}|$ grande.

Logo, P é globalmente hipoelítico.

Os dois exemplos que seguem mostram como que a soma de 으 peradores de ordem um podem afetar a não hipoeliticidade global de um certo operador diferencial parcial. 
EXEMPLO 5 :

Sejam $\quad P_{2}=\frac{\partial^{2}}{\partial x^{2}}$ e $\quad P_{1}=-i \frac{\partial}{\partial y}$.

$P_{2}$ não é globalmente hipoelítico, pois,

$\mathrm{P}_{2}|\cos y|^{3}=\frac{\partial^{2}}{\partial \mathrm{x}^{2}}|\cos y|^{3}=0 \in \mathrm{P}_{2 \pi}$

e

$$
\begin{aligned}
& |\cos y|^{3} \text { e } P_{2 \pi}^{\prime} \mid P_{2 \pi} . \\
& \text { Seja } P=P_{2}+P_{1}=\frac{\partial^{2}}{\partial x^{2}}-i \frac{\partial}{\partial y} .
\end{aligned}
$$

Afirmação: P não é globalmente hipoelítico.

De fato,

$u=\sum_{n=0}^{\infty} \cos n x e^{i n^{2} y} \in P_{2 \pi}^{\prime} \backslash P_{2 \pi}$

pois a seqüência dos coeficientes da série parcial de Fourier è de crescimento lento é não decresce rapidamente.

$$
\begin{aligned}
& P u=-\sum_{n=0}^{\infty} n^{2} \cos n x e^{i n^{2} y}-i \sum_{n=0}^{\infty} i n^{2} \cos n x e^{i n^{2} y}= \\
& =0 \in P_{2 \pi} .
\end{aligned}
$$

EXEMPLO 6 :

Sejam $\quad P_{2}=\frac{\partial^{2}}{\partial x^{2}} \quad$ e $\quad P_{1}=\alpha \frac{\partial}{\partial y} \quad, \quad \alpha \in \mathbb{R}^{*}$.

Então, $P=P_{2}+P_{1}$ é globalmente hipoelítico.

De fato,

$P(m, n)=-m^{2}+i \alpha n$ 


$$
|P(m, n)|=m^{4}+\alpha^{2} n^{2} \geqq m^{2}+\alpha^{2} n^{2} \geq \min \left\{1, \alpha^{2}\right\}|(m, n)|^{2} .
$$

- Exemplo 1 serviu para nos mostrar um operador hiperbóli co que é globalmente hipoelítico. Os Exemplos 2 e 3 mostraram que a adição de operadores de ordem 0 e 1 , respectivamente, ao-operador do Exemplo 1 pode resultar em um operador não glo balmente hipoelitico. Vejamos agora que se um operador é elíti co isto não pode acontecer, i.é., qualquer operador elítico é globalmente hipoelitico (quando somamos a um operador elítico de ordem $\mathrm{N}$ um operador de ordem menor do que $\mathrm{N}$ ○ operador re sultante será, ainda, elítico).

Seja $P=P_{m}+P_{m-1}$ um operador diferencial parcial eliti co, onde $\mathrm{P}_{\mathrm{m}}$ é homogêneo de ordem $\mathrm{m}$ e $\mathrm{P}_{\mathrm{m}-1}$ é de ordem no máximo $\mathrm{m}-1$.

Como P é elítico temos que:

$$
\inf _{|\xi|=1}\left|P_{m}(\xi)\right|=c_{0}>0 \text { e se } \xi \in \mathbb{R}^{n}
$$

então

$$
\begin{aligned}
& \left|P_{m}(\xi)\right| \geqq c_{0}|\xi|^{m} . \\
& \text { Também existe } M>0 \text { tal que } \\
& \left|P_{m-1}(\xi)\right| \leqq M|\xi|^{m-1} \quad \text { se } \quad|\xi| \geqq 1 . \\
& \text { Seja } \quad C=\max \left\{1, \frac{2 M}{C_{0}}\right\} . \\
& \text { Assim, se }|\xi| \geqq C \\
& |P(\xi)| \geqq\left|P_{m}(\xi)\right|-\left|P_{m-1}(\xi)\right| \geqq c_{0}|\xi|^{m}-\left|P_{m-1}(\xi)\right| .
\end{aligned}
$$


Mas

$$
\begin{aligned}
& -\left|\mathrm{P}_{\mathrm{m}-1}(\xi)\right| \geqq-M|\xi|^{\mathrm{m}-1}=-\frac{\mathrm{c}_{\mathrm{O}}}{2} \cdot \frac{2 \mathrm{M}}{\mathrm{c}_{\mathrm{o}}}|\xi|^{\mathrm{m}-1} \geqq \\
& \geqq-\frac{\mathrm{c}_{\mathrm{o}}}{2} \cdot \mathrm{C}|\xi|^{\mathrm{m}-1} \geqq-\frac{\mathrm{c}_{\mathrm{o}}}{2}|\xi|^{\mathrm{m}} .
\end{aligned}
$$

Logo, se $|\xi| \geqq c$ temos que

$$
|P(\xi)| \geqq \frac{c_{o}}{2}|\xi|^{m}
$$

Assim, se $\ell \in \mathbb{Z}^{n}$ e $|\ell| \geqq c$ entäo

$$
|P(\ell)| \geqq \frac{c_{0}}{2}|\ell|^{m}
$$

Logo, P é globalmente hipoelítico.

4. UM EXEMPLO DE OPERADOR DIFERENCIAL PARCIAL A COEFICIENTES

\section{VARIAVEIS GLOBALMENTE HIPOELITICO}

Sejam $\phi, a \in P_{2 \pi}(\mathbb{R})$ a valores reais tais que:

i) $\phi(0) \neq 0$

ii) $\phi(x)=0$ se $x \in\left[-\pi,-\frac{\pi}{2}\right] \cup\left[\frac{\pi}{2}, \pi\right]$.

Então o operador $\mathrm{P}$, dado por

$$
P u=-\frac{\partial^{2} u}{\partial x^{2}}-\phi^{2}(x) \frac{\partial^{2} u}{\partial y^{2}}+a(x) \frac{\partial u}{\partial y}
$$

é globalmente hipoelitico. 
Tomemos as séries parciais de Fourier, com respeito a $\mathrm{y}$, de $u$ e $f$.

$$
u=\sum_{n \in \mathbb{Z}} e_{n} u_{n} \quad \text { e } f(x, y)=\sum_{n \in \mathbb{Z}} f_{n}(x) e^{i n y}
$$

onde $u_{n}$ e $P_{2 \pi}^{\prime}(\mathbb{R})$ e $f_{n} \in P_{2 \pi}(\mathbb{R})$.

Lembremos que $\left(f_{n}^{(k)}\right)_{n \in \mathbb{Z}}$ é de decrescimento rápido pa ra todo $\mathrm{k} \in \mathbb{N}$.

Agora,

$$
\begin{aligned}
& P(u)=\sum_{n \in \mathbb{Z}}\left[-u_{n}^{\prime \prime}(x)+\left(n^{2} \phi^{2}(x)+i n a(x)\right) u_{n}(x)\right] e^{i n y}= \\
& =\sum_{n} e_{\mathbb{Z}} f_{n}(x) e^{i n y} .
\end{aligned}
$$

Logo,

$$
L u_{n}=\left[-u_{n}^{\prime \prime}+\left(n^{2} \phi^{2}(x)+i n a(x)\right) u_{n}(x)\right]=f_{n}(x)
$$

Como o operador diferencial ordinário L é hipoelítico, temos que $u_{n} \in P_{2 \pi}(\mathbb{R})$.

Para provarmos que $u \in \mathrm{P}_{2 \pi}\left(\mathbb{R}^{2}\right)$, faremos estimativas sobre o crescimento de $\left(u_{n}\right)$.

Escrevamos

$$
u_{n}(x)=u_{n}(t)+\int_{t}^{x} u_{n}^{\prime}(s) d s,
$$

onde $t \in[-\delta, \delta]$ e $\delta>0$ é tal que: se $t \in[-\delta, \delta]$ então $\phi^{2}(t) \geqq \frac{\phi^{2}(0)}{2}>0$. 


$$
\begin{aligned}
& \left|u_{n}(x)\right|^{2}=\left|u_{n}(t)\right|^{2}+\left|\int_{t}^{x} u_{n}^{\prime}(s) d s\right|^{2}+2\left|u_{n}(t)\right| . \\
& \cdot\left|\int_{t}^{x} u_{n}^{\prime}(s) d s\right| \leqq 2\left|u_{n}(t)\right|^{2}+2\left|\int_{t}^{x} u_{n}^{\prime}(s) d s\right|^{2} \leqq \\
& \leqq 2\left|u_{n}(t)\right|^{2}+2\left(\int_{t}^{x}\left|u_{n}^{\prime}(s)\right| d s\right)^{2} \leqq 2\left|u_{n}(t)\right|^{2}+ \\
& +2\left(\int_{-\pi}^{\pi}\left|u_{n}^{\prime}(s)\right| d s\right)^{2} \leqq\left|u_{n}(t)\right|^{2}+2 \int_{-\pi}^{\pi} d s \int_{-\pi}^{\pi}\left|u_{n}^{\prime}(s)\right|^{2} d s \leqq \\
& \leqq 4 \pi\left(\left|u_{n}(t)\right|^{2}+\int_{-\pi}^{\pi}\left|u_{n}^{\prime}(s)\right|^{2} d s\right) \cdot
\end{aligned}
$$

Integrando em $t$ sobre $[-\delta, \delta]$ temos:

$$
\begin{aligned}
& 2 \delta\left|u_{n}(x)\right|^{2} \leqq 4 \pi\left(\int_{-\delta}^{\delta}\left|u_{n}(t)\right|^{2} d t+2 \delta \int_{-\pi}^{\pi}\left|u_{n}^{\prime}(s)\right|^{2} d s\right) \leqq \\
& \leqq 4 \pi\left(\frac{2}{\phi^{2}(0)} \int_{-\delta}^{\delta}\left|u_{n}(t)\right|^{2} \phi^{2}(t) d t+2 \delta \int_{-\pi}^{\pi}\left|u_{n}^{\prime}(s)\right|^{2} d s\right) \leqq \\
& \leqq 4 \pi\left(\frac{2}{\phi^{2}(0)} \int_{-\pi}^{\pi}\left|u_{n}(t)\right|^{2} \phi^{2}(t) d t+2 \delta \int_{-\pi}^{\pi}\left|u_{n}^{\prime}(s)\right|^{2} d s\right) \leqq \\
& \leqq \operatorname{cte} \int_{-\pi}^{\pi}\left(\left|u_{n}(t)\right|^{2} \phi^{2}(t)+\left|u_{n}^{\prime}(t)\right|^{2}\right) .
\end{aligned}
$$

Se chamarmos

$$
\left\|u_{n}\right\|_{\phi}=\left(\int_{-\pi}^{\pi}\left(\left|u_{n}(t)\right|^{2} \phi^{2}(t)+\left|u_{n}^{i}(t)\right|^{2}\right) d t\right)^{\frac{1}{2}}
$$

então poderemos dizer que existe $C>0$ tal que

$$
\left|u_{n}(x)\right| \leqq c\left\|u_{n}\right\|_{\phi} \text {. }
$$

Se multiplicamos a equação $L u_{n}=f_{n}$ por $\bar{u}_{n}$ e integra mos sobre $[-\pi, \pi]$ obteremos: 
109

$$
\begin{aligned}
& -\int_{-\pi}^{\pi} u_{n}^{\prime \prime}(x) \overline{u_{n}}(x) d x+n^{2} \int_{-\pi}^{\pi} \phi^{2}(x)\left|u_{n}(x)\right|^{2} d x+ \\
& +\operatorname{in} \int_{-\pi}^{\pi} a(x)\left|u_{n}(x)\right|^{2} d x=\int_{-\pi}^{\pi} f_{n}(x) \overline{u_{n}}(x) d x
\end{aligned}
$$

Mas,

$$
\begin{aligned}
& \int_{-\pi}^{\pi} u_{n}^{\prime \prime}(x) \overline{u_{n}}(x) d x=\left.u_{n}^{\prime}(x) \overline{u_{n}}(x)\right|_{-\pi} ^{\pi}-\int_{-\pi}^{\pi} u_{n}^{\prime}(x) \overline{u_{n}^{\prime}(x)} d x= \\
& =\int_{-\pi}^{\pi}\left|u_{n}^{\prime}(x)\right|^{2} d x .
\end{aligned}
$$

Assim, (*) se reescreve como:

$$
\begin{aligned}
& \left\|u_{n}\right\|_{\phi}^{2}+\text { in } \int_{-\pi}^{\pi} a(x)\left|u_{n}(x)\right|^{2} d x=\int_{-\pi}^{\pi} f_{n}(x) \overline{u_{n}}(x) d x \\
& \text { Definamos }\|g\|_{2}=\left(\int_{-\pi}^{\pi}|g(x)|^{2} d x\right)^{\frac{1}{2}} \text { e observemos que }
\end{aligned}
$$

existe $C>0$ tal que

$$
\|g\|_{2}^{2}=\int_{-\pi}^{\pi}|g(x)|^{2} d x \leqq c\|g\|_{\phi}^{2} \int_{-\pi}^{\pi} d x=2 \pi c\|g\|_{\phi}^{2} .
$$

Podemos escrever

$$
\begin{aligned}
& \left\|u_{n}\right\|_{\phi}^{4} \leqq\left\|u_{n}\right\|_{\phi}^{4}+n^{2}\left(\int_{-\pi}^{\pi} a(x)\left|u_{n}(x)\right|^{2} d x\right)^{2}= \\
& =\left.\left.\left|\left\|u_{n}\right\|_{\phi}^{2}+i n \int_{-\pi}^{\pi} a(x)\right| u_{n}(x)\right|^{2} d x\right|^{2}= \\
& =\left|\int_{-\pi}^{\pi} f_{n}(x) \bar{u}_{n}(x) d x\right|^{2} \leqq\left(\int_{-\pi}^{\pi}\left|f_{n}(x)\right|^{2} d x\right)\left(\int_{-\pi}^{\pi}\left|u_{n}(x)\right|^{2} d x\right)^{2}= \\
& =\left\|f_{n}\right\|_{2}^{2}\left\|u_{n}\right\|_{2}^{2} \leqq c^{2}\left\|f_{n}\right\|_{2}^{2} \cdot\left\|u_{n}\right\|_{\phi}^{2} \text { para algum } c>0 .
\end{aligned}
$$


Logo, se $u_{n} \neq 0$,

$\left\|u_{n}\right\|_{\phi} \leqq c\left\|f_{n}\right\|_{2}$

Estamos agora em condições de mostrar que $\left(u_{n}\right)$ é de de crescimento rápido.

Seja $k \in \mathbb{N}$ então

$\left|u_{n}(x)\right| \leqq \operatorname{cte}\left\|u_{n}\right\|_{\phi} \leqq$ cte $\left\|f_{n}\right\|_{2} \leqq \frac{\text { cte }}{(1+|n|)^{k}}$.

Pois, como $f_{n}$ é de decrescimento rápido,

$\left\|f_{n}\right\|_{2}^{2}=\int_{-\pi}^{\pi}\left|f_{n}(x)\right|^{2} d x \leq \frac{c t e}{(1+|n|)^{2 k}} \int_{-\pi}^{\pi} d x=\frac{\text { cte }}{(1+|n|)^{2 k}}$.

Mostremos agora que (un ${ }_{n}^{\prime}$ é de decrescimento rápido.

Seja $\quad v_{n}=u_{n}^{\prime}$.

Então,

$L v_{n}(x)=-v_{n}^{\prime \prime}(x)+\left(n^{2} \phi^{2}(x)+i n a(x)\right) v_{n}(x)=$

$=-u_{n}^{\prime \prime \prime}(x)+\left(n^{2} \phi^{2}(x)+i n a(x)\right) u_{n}^{\prime}(x)=$

$=\frac{d}{d x}\left[-u_{n}^{\prime \prime}(x)+\left(n^{2} \phi^{2}(x)+i n a(x)\right) u_{n}(x)\right]-$

$-\left[2 n^{2} \phi(x) \phi^{\prime}(x)+i n a^{\prime}(x)\right] u_{n}(x)=$

$=f_{n}^{\prime}(x)-\left[2 n^{2} \phi(x) \phi^{\prime}(x)+i n a^{\prime}(x)\right] u_{n}(x)$.

Mas,

$\mid\left(2 n^{2} \phi(x) \phi^{\prime}(x)+i n a^{\prime}(x)|| u_{n}(x)\left|\leqq c \cdot n^{2} \cdot\right| u_{n}(x) \mid\right.$ 
e como $\left(n^{2} u_{n}\right)$ e $\left(f_{n}^{\prime}\right)$ são de decrescimento rápido, segue-se que

$$
g_{n}(x)=f_{n}^{\prime}(x)-\left[2 n^{2} \phi(x) \phi^{\prime}(x)+i n a^{\prime}(x)\right] \cdot u_{n}(x)
$$

também é de decrescimento rápido.

Logo, $v_{n}$ è tal que $L v_{n}=g_{n}$, onde $g_{n}$ é de decresci mento rápido.

Portanto, lisando o mesmo argumento que foi utilizado para mostrar que $u_{n}$ era de decrescimento rápido, concluímos que $v_{n}$ decresce rapidamente.

Para mostrar que $u_{n}^{(k)}$ é de decrescimento rápido procede remos por indução sobre $k \in \mathbb{N}$.

$$
\begin{aligned}
& \text { Notemos que, se } v_{n}=u_{n}^{(k)} \text { então } \\
& L v_{n}(x)=-u_{n}^{(k+2)}(x)+\left(n^{2} \phi^{2}(x)+i n a(x)\right) u_{n}^{(k)}(x)= \\
& =\left(\frac{d}{d x}\right)^{k}\left[-u_{n}^{\prime \prime}(x)+\left(n^{2} \phi^{2}(x)+i n a(x) u_{n}(x)\right]-\right. \\
& -\sum_{j=0}^{k-1}\left(\begin{array}{l}
k \\
j
\end{array}\right)\left(\frac{d}{d x}\right)^{k-j}\left(n^{2} \phi^{2}(x)+i n a(x)\right)\left(\frac{d}{d x}\right)^{j} u_{n}(x)= \\
& =f_{n}^{(k)}(x)-\sum_{j=0}^{k-1}\left(\begin{array}{l}
k \\
j
\end{array}\right)\left(\frac{d}{d x}\right)^{k-j}\left(n^{2} \phi^{2}(x)+i n a(x)\right)\left(\frac{d}{d x}\right)^{j} u_{n}(x)= \\
& =g_{n}(x)
\end{aligned}
$$

Se admitirmos que $\left(\frac{d}{d x}\right)^{j} u_{n}(x)$ é de decrescimento rápi do para $j=0,1, \ldots, k-1$ então $g_{n}$ também o será.

Assim $L v_{n}=g_{n}, g_{n}$ de decrescimento rápido. 
Usando as estimativas

$$
\left|v_{n}(x)\right| \leqq \operatorname{cte}\left\|v_{n}\right\|_{\phi} \leqq \operatorname{cte}\left\|g_{n}\right\|_{2} \leqq \operatorname{cte}(1+|n|)^{-p}
$$

para cada $p \in \mathbb{N}$ e todo $n \in \mathbb{N}$ concluímos que $u_{n}^{(k)}=v_{n}$ decresce rapidamente.

$$
\text { Logo, } U(x, y)=\sum_{n=-\infty}^{+\infty} u_{n}(x) e^{i n y} \text { é de classe } c^{\infty} \text {. }
$$

\section{OUTROS EXEMPLOS}

Nesta seção apresentaremos duas famílias de operadores di ferenciats parciais lineares, ambas indexadas em $[0,1]$, cujos elementos são quase sempre globalmente hipoeliticos.

\section{EXEMPLO 1 :}

Considere a família

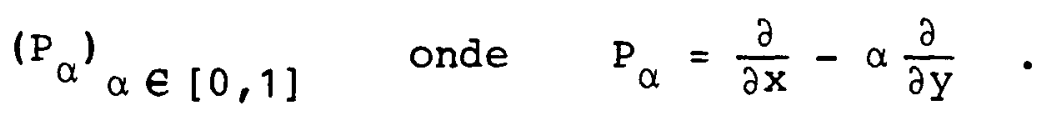

Já sabemos que $P_{\alpha}$ é globalmente hipoelitico se, e somen te se, $\alpha \in[0,1]$ é irracional não de Liouville.

Como o conjunto dos números de Liouville tem medida nula [ ver: [8] ] concluímos que $P_{\alpha}$ é globalmente hipoelítico para quase todo $\alpha$.

\section{EXEMPLO 2 :}

$$
\text { Seja } Q=\sum_{1 \leqq|\alpha| \leqq 2} a_{\alpha} D^{\alpha}, \quad a_{\alpha} \in \mathbb{R} \text {. }
$$


113

Então para quase todo a e $[0,1] \quad P=Q+a$ é globalmen te hipoelitico.

Se $(m, n) \in \mathbb{Z}^{2}$ é tal que $|Q(m, n)| \geqq 2$ então

$$
|P(m, n)|=|Q(m, n)+a| \geqq|Q(m, n)|-|a| \geq 1 .
$$

Seja

$$
A=\left\{(m, n) \in \mathbb{z}^{2} ;|Q(m, n)|<2\right\}=\left\{\left(m_{j}, n_{j}\right) \in \mathbb{Z}^{2}, j \in \mathbb{N}\right\}
$$

onde

$$
\left|m_{j}\right|+\left|n_{j}\right| \leqq\left|m_{j+1}\right|+\left|n_{j+1}\right|
$$

Considere

$$
\begin{gathered}
f_{k}(a)=\sum_{j=1}^{k} \frac{1}{\sqrt{\left|a+Q\left(m_{j}, n_{j}\right)\right|}\left(n_{j}^{2}+m_{j}^{2}\right)^{2}} \\
\text { Para cada }\left(m_{j}, n_{j}\right) \in A \text { a função } g_{j}(a)=\frac{1}{\sqrt{\left|a+Q\left(m_{j}, n_{j}\right)\right|}} \\
\text { é integrável em }[0,1] \text {, pois; se } Q\left(m_{j}, n_{j}\right) \notin[-1,0] \text { ela é con }
\end{gathered}
$$
tínua e, caso contrārio,

$$
\begin{aligned}
& \int_{0}^{1} \frac{d a}{\sqrt{\left|a+Q\left(m_{j}, n_{j}\right)\right|}}=\underset{b \rightarrow-Q\left(m_{j}, n_{j}\right)-}{\lim _{0}} \frac{d a}{\sqrt{-Q\left(m_{j}, n_{j}\right)-a}}+ \\
& +b \rightarrow-Q\left(m_{j}, n_{j}\right)+\int_{b}^{1} \frac{d a}{\sqrt{a+Q\left(m_{j}, n_{j}\right)}}= \\
& =2\left[\sqrt{-Q\left(m_{j}, n_{j}\right)}+\sqrt{1+Q\left(m_{j}, n_{j}\right)}\right] .
\end{aligned}
$$

Também, para todo $j$ vale:

$$
\left|\int_{0}^{1} \frac{d a}{\sqrt{\left|a+Q\left(m_{j}, n_{j}\right)\right|}}\right| \leqq 2\left[\sqrt{\left(Q\left(m_{j}, n_{j}\right)\right.}+\sqrt{\left|1+Q\left(m_{j}, n_{j}\right)\right|} .\right.
$$


Temos que

$f_{k}(a)+\sum_{j=1}^{\infty} \frac{1}{\sqrt{\left|a+Q\left(m_{j}, n_{j}\right)\right|}\left(m_{j}^{2}+n_{j}^{2}\right)^{2}}$

Pelo Teorema da Convergēncia Monótona

$\int_{0}^{1}\left(\sum_{j=1}^{\infty} \frac{1}{\sqrt{\left|a+Q\left(m_{j}, n_{j}\right)\right|} \cdot\left(m_{j}^{2}+n_{j}^{2}\right)^{2}}\right) d a=$

$=\sum_{j=1}^{\infty} \int_{0}^{1} \frac{d a}{\sqrt{\left|a+Q\left(m_{j}, n_{j}\right)\right|}\left(m_{j}^{2}+n_{j}^{2}\right)^{2}} \leqq$

$\leqq 2 \sum_{j=1}^{\infty} \frac{\sqrt{\left|1+Q\left(m_{j} n_{j}\right)\right|}+\sqrt{\left|Q\left(m_{j}, n_{j}\right)\right|}}{\left(m_{j}^{2}+n_{j}^{2}\right)^{2}} \leqq$

$\leqq 2(\sqrt{3}+\sqrt{2}) \sum \frac{1}{\left(m_{j}^{2}+n_{j}^{2}\right)^{2}} \leqq 2(\sqrt{3}+\sqrt{2}) \sum_{(m, n)} \in \mathbb{Z}^{2} \frac{1}{\left(m^{2}+n^{2}\right)^{2}}<\infty$.

Pois,

$\left|Q\left(m_{j}, n_{j}\right)\right|<2$ e $\quad\left|1+Q\left(m_{j}, n_{j}\right)\right|<3$.

Assim, para quase todo a $\in[0,1]$ a série

$\sum_{j=1}^{\infty} \frac{1}{\sqrt{\left|a+Q\left(m_{j}, n_{j}\right)\right|}\left(m_{j}^{2}+n_{j}^{2}\right)^{2}}$

é finita.

Logo, existe $M_{a}>0$ tal que

$\frac{1}{\sqrt{\left|a+Q\left(m_{j} n_{j}\right)\right|}\left(m_{j}^{2}+n_{j}^{2}\right)^{2}} \leqq M_{a}$. 
115

ou seja,

$$
\begin{aligned}
& \left|P\left(m_{j}, n_{j}\right)\right|=\left|a+Q\left(m_{j}, n_{j}\right)\right| \geq \frac{1}{M_{a}^{2}\left(m_{j}^{2}+n_{j}^{2}\right)^{4}} \cdot \\
& \text { Seja } L_{a}=\left(\max \left\{M_{a}^{2}, 1\right\}\right)^{-1} \text {. Então, se }(m, n) \neq(0,0) \\
& |P(m, n)| \geqq 1 \geqq L_{a}|(m, n)|^{-8} \text { se }(m, n) \notin A \\
& |P(m, n)| \geqq L_{a}|(m, n)|^{-8} \text { se }(m, n) \in A .
\end{aligned}
$$

Logo,

$$
|P(m, n)| \geq L_{a}|(m, n)|^{-8}, \quad \forall(m, n) \in z^{2} \mid\{(0,0)\} .
$$




\section{A P ÉNDICE}

Vimos na seção 5 do Capítulo I que $s=s\left(\mathbb{Z}^{n} ; \mathbb{C}\right)$ é isomor fo a $\quad \mathrm{P}_{T}=\mathrm{P}_{\mathrm{T}}\left(\mathbb{R}^{\mathrm{n}}\right)$ e que $\mathrm{s}^{\prime}=\mathrm{s}^{\prime}\left(\mathbb{Z}^{\mathrm{n}} ; \mathbb{C}\right)$ é isomorfo a $\quad P_{T}^{\prime}=P_{T}^{\prime}\left(\mathbb{R}^{n}\right)$. Neste apēndice mostraremos que $s$ é homeo morfo a $\mathrm{P}_{\mathrm{T}}$ bem como $\mathrm{s}^{\prime}$ a $\mathrm{P}_{\mathrm{T}}^{\prime}$.

$$
\begin{aligned}
& \text { Consideraremos em } s \text { as semi-normas } \\
& \mathrm{p}_{\mathrm{k}}(\mathrm{c})=\sup _{\mathrm{m} \in \mathbb{Z}^{\mathrm{n}}}(1+|\mathrm{m}|)^{\mathrm{k}}\left|\mathrm{c}_{\mathrm{m}}\right|
\end{aligned}
$$

onde $c=\left(c_{m}\right)$ e $s$ e $k=0,1,2, \ldots$.

s é metrizável através da distāncia

$$
a(c, b)=\sum_{k=0}^{\infty} \frac{p_{k}(c-b)}{2^{k}\left(1+p_{k}(c-b)\right)}
$$

Em $P_{\mathrm{T}}$ usaremos as semi-normas usuais

$$
P_{k}(\phi)=\sup _{x \in \mathbb{R}^{n}}\left|D^{\alpha} \phi(x)\right|
$$

Considere o isomorfismo algébrico $A: S \rightarrow P_{T}$ dado por $A(c)=\sum_{m} \mathbb{Z}^{n} c_{m} e_{m}$.

$$
\text { Seja }\left(c_{j}\right)_{j \in \mathbb{N}} \text { uma seqüēncia em } s, \quad \text { onde }
$$
$c_{j}=\left(c_{m, j}\right)_{m e ~} \mathbb{z}^{n}$, tal que $c_{j} \rightarrow 0$ em $s, i . e ́ .$, para cada $k \in \mathbb{N} \quad p_{k}\left(c_{j}\right) \rightarrow 0 \quad$ quando $j \rightarrow \infty$. 
117

Sejam $E>0$ e $k$ e $\mathbb{N}$.

Assim, se $|\alpha| \leqq k$

$$
\begin{aligned}
& \left|D^{\alpha} A\left(c_{j}\right)\right|=\left|\sum_{m e} \mathbb{Z}^{n} m^{\alpha} c_{m, j} e_{m}\right| \leqq \\
& \leq \sum_{m e \mathbb{z}^{n}}\left|m^{\alpha}\right|\left|c_{m, j}\right| \leqq c t e \sum_{m} \mathbb{z}^{n}(1+|m|)^{k}\left|c_{m, j}\right| .
\end{aligned}
$$

Porém, existe $j_{0} \in \mathbb{N}$ tal que se $j \geqq j_{0}$ então

$$
(1+|m|)^{n+1+k}\left|c_{m, j}\right| \leqq p_{n+1+k}\left(c_{j}\right)<\varepsilon .
$$

Logo, para $j \geqq j_{0}$

$$
\left|D^{\alpha} A\left(c_{j}\right)\right| \leqq \text { cte } \varepsilon \cdot \sum_{m \in \mathbb{Z}^{n}} \frac{1}{(1+|m|)^{n+1}}
$$

Portanto, $P_{k}\left(A\left(C_{j}\right)\right) \rightarrow 0$ quando $j \rightarrow \infty$. Assim, A é contínuo.

Mostremos agora que

$$
\begin{aligned}
\mathrm{A}^{-1}: \mathrm{P}_{\mathrm{T}} & \rightarrow \mathrm{s} \\
\phi & \longmapsto\left(\mathrm{c}_{\mathrm{m}}(\phi)\right) \quad \text { é contínuo. }
\end{aligned}
$$

Seja $\left(\phi_{j}\right)$ uma seqüência de $P_{T}$ tal que $\phi_{j} \rightarrow 0$ em $P_{T} \cdot$ Para cada $\alpha$ e $\mathbb{N}^{\mathrm{n}}$ temos

$$
\begin{aligned}
& \left|m^{\alpha} c_{m}\left(\phi_{j}\right)\right|=\left|\frac{1}{T^{n}} \int_{[0, T]^{n}} \phi_{j}(x) m^{\alpha} e^{i w m x} d x\right|= \\
& =\frac{1}{T^{n}|\alpha|}\left|\int_{[0, T]^{n}} \phi_{j}(x) D^{\alpha} e^{i w m x} d x\right|=
\end{aligned}
$$




$$
\begin{aligned}
& =\frac{1}{T^{n}|\alpha|}\left|\int_{[0, T]^{n}} D^{\alpha} \phi_{j}(x) \cdot e^{i w m x} d x\right| \leqq \\
& \leqq \frac{1}{T^{n}{ }_{w}|\alpha|} \int_{[0, T]^{n}}\left|D^{\alpha} \phi_{j}(x)\right| d x \leqq \\
& \leqq \frac{1}{{ }_{w}|\alpha|} \sup \left|D^{\alpha} \phi_{j}(x)\right| \\
& \text { Assim, se }|\alpha| \leqq k \\
& (1+|m|)^{k}\left|c_{m}\left(\phi_{j}\right)\right|=\sum_{\ell=0}^{k}\left(\begin{array}{l}
k \\
l
\end{array}\right)|m|^{\ell}\left|c_{m}\left(\phi_{j}\right)\right|= \\
& =\sum_{\ell=0}^{k}\left(\begin{array}{l}
k \\
\ell
\end{array}\right)\left(\sum_{|\beta|=\ell} \frac{\ell !}{\beta !}\left|m^{\beta}\right|\right)\left|c_{m}\left(\phi_{j}\right)\right|= \\
& =\sum_{\ell=0}^{k}|\beta|_{=\ell}\left(\begin{array}{l}
k \\
\ell
\end{array}\right) \frac{\ell !}{\beta !}\left|m^{\beta} c_{m}\left(\phi_{j}\right)\right| \leqq \\
& \leqq \sum_{\ell=0}^{k}|\beta|^{L}=\ell\left(\begin{array}{l}
k \\
\ell
\end{array}\right) \frac{\ell !}{\beta !} \frac{1}{w|\beta|} \sup \left|D^{\beta} \phi_{j}\right| \rightarrow 0
\end{aligned}
$$

quando $j+\infty$.

Logo, $p_{k}\left(A^{-1}\left(\phi_{j}\right)\right) \rightarrow 0$ quando $j \rightarrow \infty$ e, portanto, $A^{-1}$ é continuo.

Antes de mostrarmos que $s^{\prime}$ é homeomorfo a $P_{T}^{\prime}$ mostremos que $s^{\prime}$ é homeomorfo a $s^{*}$, o dual de $s$.

Considere a aplicação

$$
\begin{aligned}
L: s^{\prime} & \rightarrow s^{*} \\
c & \longmapsto L(c)
\end{aligned}
$$


dada por $\langle\mathrm{L}(\mathrm{c}), \mathrm{d}\rangle=\sum_{\mathrm{m} \in \mathrm{z}^{\mathrm{n}}} \mathrm{c}_{\mathrm{m}} \mathrm{d}_{\mathrm{m}}$ onde $\mathrm{c}=\left(c_{\mathrm{m}}\right) \in \mathrm{s}^{\prime} \quad \mathrm{e}$ $d=\left(d_{m}\right)$ e $s$

I (c) está bem definido pois existe $c>0$ e ke IN tais que $\left|c_{m}\right| \leqq c(1+|m|)^{k}$. Também existe $C^{\prime}>0$ tal que $\left|d_{m}\right| \leqq c^{\prime}(1+|m|)^{-k-n-1}$.

Logo, $\left|c_{m} a_{m}\right| \leq c \cdot c^{\prime}(1+|m|)^{-n-1}$.

Éfácil verificar que $I$ é linear.

I é sobrejetora pois, se $f \in s^{*}$ considere $c_{m}=\left\langle f, \tilde{e}_{m}\right\rangle$ e $c=\left(c_{m}\right)$, onde $\tilde{e}_{m}=\left(\tilde{e}_{m, j}\right)$ e $\tilde{e}_{m, j}=\left\{\begin{array}{lll}1 & \text { se } & m=j \\ 0 & \text { se } & m \neq j\end{array}\right.$.

Como $f e s^{*}, i . e ́ ., f: s \rightarrow \mathbb{C}$ é linear e contínuo exis te semi-norma $p_{k}$ de $s$ tal que $|\langle f, d\rangle| \leqq c$. $p_{k}(d)$ para $c>0$ para toda d e $\mathbf{s}$.

Assim,

$$
\begin{aligned}
& \left|c_{m}\right|=\left|<f, \tilde{e}_{m}\right\rangle \mid \leqq c \cdot p_{k}\left(\tilde{e}_{m}\right)=c \cdot \sup _{j \in \mathbb{N}^{n}}(1+|j|)^{k} . \\
& \cdot\left|\tilde{e}_{m, j}\right|=c \cdot(1+|m|)^{k} .
\end{aligned}
$$

Logo $c \in s^{\prime} e$

$\langle L(c) ; d\rangle=\sum_{m \in \mathbb{Z}^{n}} c_{m} d_{m}=\sum_{m \in \mathbb{Z}^{n}}\left\langle f, \tilde{e}_{m}\right\rangle d m=\langle f, d\rangle$.

I. é injetora pois, se $c \in s^{\prime}$ e $c=\left(c_{m}\right) \neq 0$ então $c_{m} \neq 0$ para algum $m \in \mathbb{Z}^{n}$. Assim,

$\left\langle L(c), \tilde{e}_{m}>=c_{m} \neq 0\right.$, i.é., $L(c) \neq 0$.

Portanto, L é um isomorfismo entre $s^{\prime} e s^{*}$. 
121

$$
\begin{aligned}
& \left|\left(\tilde{B}\left(f_{j}\right), \theta\right)\right|=\mid\left(\sum_{m \in \mathbb{Z}^{n}}\left\langle f_{j}, \tilde{e}_{m}>e_{m}, \theta\right) \mid=\right. \\
& =\left|\sum_{m} e_{\mathbb{Z}^{n}} f_{m, j}\left(e_{m}, \theta\right)\right|=T^{n}\left|\sum_{m \mathbb{Z}^{n}} f_{m, j} c_{m}(\theta)\right|= \\
& =T^{n} q_{d}\left(f_{j}\right) \rightarrow 0
\end{aligned}
$$

quando $j \rightarrow \infty$.

Logo, $Q_{\theta}\left(\tilde{B}\left(f_{j}\right)\right) \rightarrow 0$ quando $j \rightarrow \infty$ e, portanto, $\tilde{B}$ é contínuo.

Mostremos que

$$
\begin{aligned}
\tilde{B}^{-1}: P_{\mathrm{T}}^{\prime} & \rightarrow \mathrm{s}^{*} \\
\mathrm{u} & \mapsto \mathrm{f}
\end{aligned}
$$

onde, para cada $d=\left(d_{m}\right)$ e $s$

$$
\left\langle\tilde{B}^{-1}(u), d\right\rangle=\sum_{m \in \mathbb{Z}^{n}} c_{m}(u) d_{m} \quad \text { é contíruo. }
$$

Seja $u_{j} \rightarrow 0$ em $P_{T}^{\prime}$.

Para cada de $\mathrm{s}$ existe $\theta$ e $\mathrm{P}_{\mathrm{T}}$ tal que $\mathrm{d}_{\mathrm{m}}=\mathrm{c}_{\mathrm{m}}(\theta)$.

Assim,

$$
\begin{aligned}
& q_{d}\left(\tilde{B}^{-1}\left(u_{j}\right)\right)=\left|\sum_{m \in \mathbb{Z}^{n}} c_{m}\left(u_{j}\right) d_{m}\right|= \\
& =T^{n}\left|\sum_{m} \sum_{\mathbb{Z}^{n}} c_{m}\left(u_{j}\right)\left(\theta, e_{m}\right)\right|= \\
& =T^{n}\left|\left(\sum_{m \in \mathbb{Z}^{n}} c_{m}\left(u_{j}\right) e_{m}, \theta\right)\right|=T^{n}\left|\left(u_{j}, \theta\right)\right|= \\
& =T^{n} Q_{\theta}\left(u_{j}\right) \rightarrow 0 \quad \text { quando } j \rightarrow \infty .
\end{aligned}
$$

Logo, $\tilde{B}^{-1}$ é contínuo. 


\section{B I B L I O G R A F I A}

[ 1 ] DUGUNDJI, J. - "Topology", Allyn and Bacon, Inc., Boston.

[ 2 ] EDWARDS, R.E. - "Fourier Series - A Modern Introduction", vol. I,II, Holt, Rinehart and Winton, Inc., EUA, 1967.

[ 3 ] FIGUEIREDO, D.G. - "Nümeros Irracionais e Transcendentes", IMPA, Rio de Janeiro, 1985.

[ 4 ] FUJIWARA, D. and OMORI, H. - "An Example of a Globally Hypoelliptic Operator" , Hokkaido Mathematical Journal, 12 (1983), 293-297.

[ 5 ] GREENFIELD, S.J. and WALLACH, N.R.-"Global Hipoellipticity and Liouville Numbers",Proc. Amer. Math. Soc., 31 (1972), $112-114$

[6] GRIfFIN, H. - "Elementary Theory of Numbers", Mc. Graw-Hill Book Co., N. York, 1954, 172-176.

[ 7 ] HounIE, J. - "Teoria Elementar das Distribuições", 129 Co lóquio Brasileiro de Matemática, IMPA, Rio de Janeiro, 1979 .

[ 8 ] MAÑE, R. - "Introdução à Teoria Ergódica", Projeto Eucli des, IMPA, Rio de Janeiro, 1984.

[ 9 ] SANTOS NETO, C. - "Distribuições Periódicas e Hipoeliticí dade", Dissertação de Mestrado, ICMSC, 1985.

[10] SCHWARTZ, L. - "Méthodes Mathématiques pou les sciences physiques", 2a ed., Hermann, Paris, 1965.

[11] ZEMANIAN, A.H. - "Distribution Theory and Transform Analy sis", MC. Graw-Hill, N. York. 